\title{
Charmed baryons circa 2015
}

\author{
Hai-Yang Cheng \\ Institute of Physics, Academia Sinica, Taipei \\ E-mail: phcheng@phys.sinica.edu.tw
}

Received August 27, 2015; accepted September 8, 2015

\begin{abstract}
This is essentially an update of Ref. [1] [H. Y. Cheng, Int. J. Mod. Phys. A 24 (Suppl. 1), 593 (2009)], a review of charmed baryon physics around 2007. Topics covered in this review include the spectroscopy, strong decays, lifetimes, nonleptonic and semileptonic weak decays, and electromagnetic decays of charmed baryons.
\end{abstract}

Keywords charmed baryons, spectroscopy, decays of baryons, chiral Lagrangian

PACS numbers 14.20.Lq, 13.30.-a, 12.39.Fe

\section{Contents}

1 Introduction

2 Spectroscopy

2.1 Singly charmed baryons
2.1.1 $\Lambda_{c}$ states
2.1.2 $\Sigma_{c}$ states
2.1.3 $\Xi_{c}$ states
2.1.4 $\Omega_{c}$ states

2.2 Doubly charmed baryons

3 Strong decays

3.1 Strong decays of $s$-wave charmed baryons

3.2 Strong decays of $p$-wave charmed baryons

4 Lifetimes

4.1 Singly charmed baryons

4.2 Doubly charmed baryons

5 Hadronic weak decays

5.1 Nonleptonic decays

5.2 Discussion

5.2.1 $\Lambda_{c}^{+}$decays

$5.2 .2 \quad \Xi_{c}^{+}$decays

$5.2 .3 \quad \Xi_{c}^{0}$ decays

$5.2 .4 \Omega_{c}^{0}$ decays

5.3 Charm-flavor-conserving nonleptonic decays 16

5.4 Semileptonic decays

6 Electromagnetic and weak radiative decays

6.1 Electromagnetic decays

6.2 Weak radiative decays

7 Conclusions

Acknowledgements

References

1

2

2

4

6

6

6

*Special Topic: Potential Physics at a Super $\tau$-Charm Factory (Ed. Hai-Bo Li).

\section{Introduction}

Charm baryon spectroscopy provides an excellent ground for studying the dynamics of light quarks in the environment of a heavy quark. In the past decade, many new excited charmed baryon states have been discovered by BaBar, Belle, CLEO and LHCb. $B$ decays and the $e^{+} e^{-} \rightarrow c \bar{c}$ continuum are both very rich sources of charmed baryons. Many efforts have been made to identify the quantum numbers of these new states and understand their properties.

Consider the strong decays $\Sigma_{Q} \rightarrow \Lambda_{Q} \pi$ and $\Xi_{Q}^{*} \rightarrow$ $\Xi_{Q} \pi$, where $Q=c, b$. The mass differences between $\Sigma_{c}$ and $\Lambda_{c}$ and between $\Xi_{c}{ }^{*}$ and $\Xi_{c}$ in the charmed baryon sector were found to be large enough that the strong decays of $\Sigma_{c}$ and $\Xi_{c}^{*}$ are kinematically allowed. Consequently, the charmed baryon system offers a unique and excellent laboratory for testing the ideas and predictions of heavy quark symmetry of the heavy quark and chiral symmetry of the light quarks. These tests will have interesting implications for the low-energy dynamics of heavy baryons interacting with Goldstone bosons.

Theoretical interest in hadronic weak decays of charmed baryons peaked around the early 1990s and then faded away. To date, we still do not have a good phenomenological model, not mentioning the quantum chromodynamics (QCD)-inspired approach as in meson decays, to describe the complicated physics of baryon decays. We need cooperative efforts from both experimentalists and theorists to make progress in this arena.

This review is essentially an update of Ref. [1], which described charmed baryon physics around 2007. The outline of the content is the same as that of Ref. [1] except

(C) The Author(s) 2015. This article is published with open access at www.springer.com/11467 and journal.hep.com.cn/fop 
that we add discussions of the spectroscopy and lifetimes of doubly charmed baryons.

Several excellent review articles on charmed baryons can be found in Refs. [2-7].

\section{Spectroscopy}

\subsection{Singly charmed baryons}

The singly charmed baryon is composed of a charmed quark and two light quarks. Each light quark is a triplet of flavor $\mathrm{SU}(3)$. There are two different SU(3) multiplets of charmed baryons: a symmetric sextet $\mathbf{6}$ and an antisymmetric antitriplet $\overline{\mathbf{3}}$. The $\Lambda_{c}^{+}, \Xi_{c}^{+}$and $\Xi_{c}^{0}$ form a $\overline{\mathbf{3}}$ representation and they all decay weakly. The $\Omega_{c}^{0}$, $\Xi_{c}^{\prime+}, \Xi_{c}^{\prime 0}$ and $\Sigma_{c}^{++,+, 0}$ form a 6 representation; among them, only $\Omega_{c}^{0}$ decays weakly. We follow the Particle Data Group's (PDG's) convention [8] of using a prime to distinguish the $\Xi_{c}$ in the $\mathbf{6}$ from that in the $\overline{\mathbf{3}}$.

In the quark model, the orbital angular momentum of the light diquark can be decomposed into $\boldsymbol{L}_{\ell}=\boldsymbol{L}_{\rho}+\boldsymbol{L}_{\lambda}$, where $\boldsymbol{L}_{\rho}$ is the orbital angular momentum between the two light quarks, and $\boldsymbol{L}_{\lambda}$ is the orbital angular momentum between the diquark and the charmed quark. Although the separate spin angular momentum $S_{\ell}$ and orbital angular momentum $L_{\ell}$ of the light degrees of freedom are not well defined, they are included for guidance from the quark model. In the heavy quark limit, the spin of the charmed quark $\boldsymbol{S}_{c}$, and the total angular momentum of the two light quarks $\boldsymbol{J}_{\ell}=\boldsymbol{S}_{\ell}+\boldsymbol{L}_{\ell}$, are separately conserved. The total angular momentum is given by $\boldsymbol{J}=\boldsymbol{S}_{c}+\boldsymbol{J}_{\ell}$. It is convenient to use $S_{\ell}$, $L_{\ell}$, and $J_{\ell}$ to enumerate the spectrum of states. Moreover, one can define two independent relative momenta, $\boldsymbol{p}_{\rho}=\frac{1}{\sqrt{2}}\left(\boldsymbol{p}_{1}-\boldsymbol{p}_{2}\right)$ and $\boldsymbol{p}_{\lambda}=\frac{1}{\sqrt{6}}\left(\boldsymbol{p}_{1}+\boldsymbol{p}_{2}-2 \boldsymbol{p}_{c}\right)$, from the two light quark momenta $\boldsymbol{p}_{1}, \boldsymbol{p}_{2}$ and the heavy quark momentum $\boldsymbol{p}_{c}$. Denoting the quantum numbers $L_{\rho}$ and $L_{\lambda}$ as the eigenvalues of $\boldsymbol{L}_{\rho}^{2}$ and $\boldsymbol{L}_{\lambda}^{2}$, respectively, the $\rho$-orbital momentum $L_{\rho}$ describes relative orbital exci-

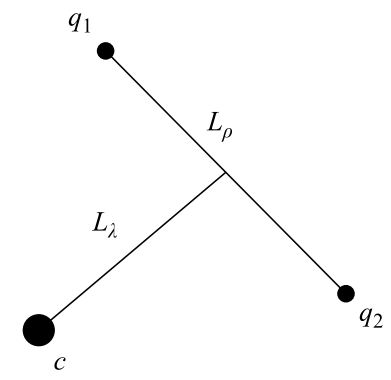

Fig. 1 Singly charmed baryon where $L_{\rho}$ describes relative orbital excitation of the two light quarks and $L_{\lambda}$ the orbital excitation of the center of the mass of the two light quarks relative to the charmed quark.

tations of the two light quarks, and the $\lambda$-orbital momentum $L_{\lambda}$ describes orbital excitations of the center of mass of the two light quarks relative to the heavy quark (see Fig. 1). The $p$-wave heavy baryon can be in either the $\left(L_{\rho}=0, L_{\lambda}=1\right) \lambda$-state or the $\left(L_{\rho}=1, L_{\lambda}=0\right) \rho$ state. The orbital $\lambda$-state ( $\rho$-state) is clearly symmetric (antisymmetric) under the interchange of $\boldsymbol{p}_{1}$ and $\boldsymbol{p}_{2}$. In the following, we use the notation $\mathcal{B}_{c J_{\ell}}\left(J^{P}\right)\left(\tilde{\mathcal{B}}_{c J_{\ell}}\left(J^{P}\right)\right)$ to denote the states that are symmetric (antisymmetric) in the orbital wave functions under the exchange of two light quarks. The lowest-lying orbitally excited baryon states are the $p$-wave charmed baryons, the quantum numbers of which are listed in Table 1.

The next orbitally excited states are the positiveparity excitations with $L_{\rho}+L_{\lambda}=2$. There exist multiplets (e.g., $\Lambda_{c 2}$ and $\hat{\Lambda}_{c 2}$ ) with the symmetric orbital wave function, corresponding to $L_{\lambda}=2, L_{\rho}=0$ and $L_{\lambda}=0, L_{\rho}=2$ (see Table 2). We use a hat to distinguish them. Because the orbital $L_{\lambda}=L_{\rho}=1$ states are antisymmetric under the interchange of two light quarks, we use a tilde to denote them. Moreover, the notation $\tilde{\mathcal{B}}_{c J_{\ell}}^{L_{\ell}}\left(J^{P}\right)$ is reserved for tilde states in the $\overline{\mathbf{3}}$, as the quantum number $L_{\ell}$ is needed to distinguish different states.

The observed mass spectra and decay widths of charmed baryons are summarized in Table 3 (see also Fig. 2). Note that except for the parity of the lightest $\Lambda_{c}^{+}$and the heavier one $\Lambda_{c}(2880)^{+}$, none of the other $J^{P}$

Table 1 The $p$-wave charmed baryons denoted by $\mathcal{B}_{c J_{\ell}}\left(J^{P}\right)$ and $\tilde{\mathcal{B}}_{c J_{\ell}}\left(J^{P}\right)$ where $J_{\ell}$ is the total angular momentum of the two light quarks. The orbital $\rho$-states with $L_{\rho}=1$ and $L_{\lambda}=0$ have odd orbital wave functions under the permutation of the two light quarks and are denoted by a tilde.

\begin{tabular}{|c|c|c|c|c|c|c|c|c|c|}
\hline State & $\mathrm{SU}(3)$ & $S_{\ell}$ & $L_{\ell}\left(L_{\rho}, L_{\lambda}\right)$ & $J_{\ell}^{P_{\ell}}$ & State & $\mathrm{SU}(3)$ & $S_{\ell}$ & $L_{\ell}\left(L_{\rho}, L_{\lambda}\right)$ & $J_{\ell}^{P_{\ell}}$ \\
\hline$\Lambda_{c 1}\left(\frac{1}{2}^{-}, \frac{3}{2}^{-}\right)$ & $\overline{3}$ & 0 & $1(0,1)$ & $1^{-}$ & $\Sigma_{c 0}\left(\frac{1}{2}^{-}\right)$ & 6 & 1 & $1(0,1)$ & $0^{-}$ \\
\hline$\tilde{\Lambda}_{c 0}\left(\frac{1}{2}^{-}\right)$ & $\overline{3}$ & 1 & $1(1,0)$ & $0^{-}$ & $\Sigma_{c 1}\left(\frac{1}{2}^{-}, \frac{3}{2}^{-}\right)$ & 6 & 1 & $1(0,1)$ & $1^{-}$ \\
\hline$\tilde{\Lambda}_{c 2}\left(\frac{3}{2}^{-}, \frac{5}{2}^{-}\right)$ & $\overline{3}$ & 1 & $1(1,0)$ & $2^{-}$ & $\tilde{\Sigma}_{c 1}\left(\frac{1}{2}^{-}, \frac{3}{2}^{-}\right)$ & 6 & 0 & $1(1,0)$ & $1^{-}$ \\
\hline$\Xi_{c 1}\left(\frac{1}{2}^{-}, \frac{3}{2}^{-}\right)$ & $\overline{3}$ & 0 & $1(0,1)$ & $1^{-}$ & $\Xi_{c 0}^{\prime}\left(\frac{1}{2}^{-}\right)$ & 6 & 1 & $1(0,1)$ & $0^{-}$ \\
\hline$\tilde{\Xi}_{c 0}\left(\frac{1}{2}^{-}\right)$ & $\overline{3}$ & 1 & $1(1,0)$ & $0^{-}$ & $\Xi_{c 1}^{\prime}\left(\frac{1}{2}^{-}, \frac{3}{2}^{-}\right)$ & 6 & 1 & $1(0,1)$ & $1^{-}$ \\
\hline$\tilde{\Xi}_{c 2}\left(\frac{3}{2}^{-}, \frac{5}{2}^{-}\right)$ & $\overline{3}$ & 1 & $1(1,0)$ & $2^{-}$ & $\tilde{\Xi}_{c 1}^{\prime}\left(\frac{1}{2}^{-}, \frac{3}{2}^{-}\right)$ & 6 & 0 & $1(1,0)$ & $1^{-}$ \\
\hline
\end{tabular}


Table 2 The first positive-parity excitations of charmed baryons denoted by $\mathcal{B}_{c J_{\ell}}\left(J^{P}\right), \hat{\mathcal{B}}_{c J_{\ell}}\left(J^{P}\right)$ and $\tilde{\mathcal{B}}_{c J_{\ell}}^{L_{\ell}}\left(J^{P}\right)$. Orbital $L_{\rho}=L_{\lambda}=1$ states with antisymmetric orbital wave functions are denoted by a tilde. States with the symmetric orbital wave functions $L_{\rho}=2$ and $L_{\lambda}=0$ are denoted by a hat. For convenience, we drop the superscript $L_{\ell}$ for tilde states in the sextet.

\begin{tabular}{|c|c|c|c|c|c|c|c|c|c|}
\hline State & $\mathrm{SU}(3)$ & $S_{\ell}$ & $L_{\ell}\left(L_{\rho}, L_{\lambda}\right)$ & $J_{\ell}^{P_{\ell}}$ & State & $\mathrm{SU}(3)$ & $S_{\ell}$ & $L_{\ell}\left(L_{\rho}, L_{\lambda}\right)$ & $J_{\ell}^{P_{\ell}}$ \\
\hline$\Lambda_{c 2}\left(\frac{3}{2}^{+}, \frac{5}{2}^{+}\right)$ & $\overline{\mathbf{3}}$ & 0 & $2(0,2)$ & $2^{+}$ & $\Sigma_{c 1}\left(\frac{1}{2}^{+}, \frac{3}{2}^{+}\right)$ & 6 & 1 & $2(0,2)$ & $1^{+}$ \\
\hline$\hat{\Lambda}_{c 2}\left(\frac{\overline{3}}{2}^{+}, \frac{\overline{5}}{2}^{+}\right)$ & $\overline{3}$ & 0 & $2(2,0)$ & $2^{+}$ & $\Sigma_{c 2}\left(\frac{3}{2}^{+}, \frac{\overline{5}}{2}^{+}\right)$ & 6 & 1 & $2(0,2)$ & $2^{+}$ \\
\hline$\tilde{\Lambda}_{c 0}^{1}\left(\frac{1}{2}^{+}\right)$ & $\overline{3}$ & 1 & $1(1,1)$ & $0^{+}$ & $\hat{\Sigma}_{c 1}\left(\frac{1}{2}^{+}, \frac{3}{2}^{+}\right)$ & 6 & 1 & $2(2,0)$ & $1^{+}$ \\
\hline$\tilde{\Lambda}_{c 1}^{1}\left(\frac{1}{2}^{+}, \frac{3}{2}^{+}\right)$ & $\overline{3}$ & 1 & $1(1,1)$ & $1^{+}$ & $\hat{\Sigma}_{c 2}\left(\frac{3}{2}^{+}, \frac{5}{2}^{+}\right)$ & 6 & 1 & $2(2,0)$ & $2^{+}$ \\
\hline$\tilde{\Lambda}_{c 2}^{1}\left(\frac{3}{2}^{+}, \frac{5}{2}^{+}\right)$ & $\overline{3}$ & 1 & $1(1,1)$ & $2^{+}$ & $\hat{\Sigma}_{c 3}\left(\frac{5}{2}^{+}, \frac{7}{2}^{+}\right)$ & 6 & 1 & $2(2,0)$ & $3^{+}$ \\
\hline$\tilde{\Lambda}_{c 2}^{2}\left(\frac{3}{2}^{+}, \frac{5}{2}^{+}\right)$ & $\overline{3}$ & 1 & $2(1,1)$ & $2^{+}$ & $\tilde{\Sigma}_{c 1}\left(\frac{1}{2}^{+}, \frac{3}{2}^{+}\right)$ & 6 & 0 & $1(1,1)$ & $1^{+}$ \\
\hline$\tilde{\Lambda}_{c 3}^{2}\left(\frac{5}{2}^{+}, \frac{7}{2}^{+}\right)$ & $\overline{3}$ & 1 & $2(1,1)$ & $3^{+}$ & $\tilde{\Sigma}_{c 2}\left(\frac{3}{2}^{+}, \frac{5}{2}^{+}\right)$ & 6 & 0 & $2(1,1)$ & $2^{+}$ \\
\hline$\Xi_{c 2}\left(\frac{3}{2}^{+}, \frac{5}{2}^{+}\right)$ & $\overline{3}$ & 0 & $2(0,2)$ & $2^{+}$ & $\Xi_{c 1}^{\prime}\left(\frac{1}{2}^{+}, \frac{3}{2}^{+}\right)$ & 6 & 1 & $2(0,2)$ & $1^{+}$ \\
\hline$\hat{\Xi}_{c 2}\left(\frac{3}{2}^{+}, \frac{5}{2}^{+}\right)$ & $\overline{3}$ & 0 & $2(2,0)$ & $2^{+}$ & $\Xi_{c 2}^{\prime}\left(\frac{3}{2}^{+}, \frac{5}{2}^{+}\right)$ & 6 & 1 & $2(0,2)$ & $2^{+}$ \\
\hline$\tilde{\Xi}_{c 1}\left(\frac{1}{2}^{+}, \frac{3}{2}^{+}\right)$ & $\overline{3}$ & 1 & $0(1,1)$ & $1^{+}$ & $\Xi_{c 3}^{\prime}\left(\frac{5}{2}^{+}, \frac{7}{2}^{+}\right)$ & 6 & 1 & $2(0,2)$ & $3^{+}$ \\
\hline$\tilde{\Xi}_{c 1}^{2}\left(\frac{1}{2}^{+}, \frac{3}{2}^{+}\right)$ & $\overline{3}$ & 1 & $2(1,1)$ & $1^{+}$ & $\tilde{\Xi}_{c 0}^{\prime}\left(\frac{1}{2}^{+}\right)$ & 6 & 0 & $0(1,1)$ & $0^{+}$ \\
\hline$\tilde{\Xi}_{c 2}^{2}\left(\frac{3}{2}^{+}, \frac{5}{2}^{+}\right)$ & $\overline{3}$ & 1 & $2(1,1)$ & $2^{+}$ & $\tilde{\Xi}_{c 1}^{\prime}\left(\frac{1}{2}^{+}, \frac{3}{2}^{+}\right)$ & 6 & 0 & $1(1,1)$ & $1^{+}$ \\
\hline$\tilde{\Xi}_{c 3}^{2}\left(\frac{5}{2}^{+}, \frac{7}{2}^{+}\right)$ & $\overline{3}$ & 1 & $2(1,1)$ & $3^{+}$ & $\tilde{\Xi}_{c 2}^{\prime}\left(\frac{3}{2}^{+}, \frac{5}{2}^{+}\right)$ & 6 & 0 & $2(1,1)$ & $2^{+}$ \\
\hline
\end{tabular}
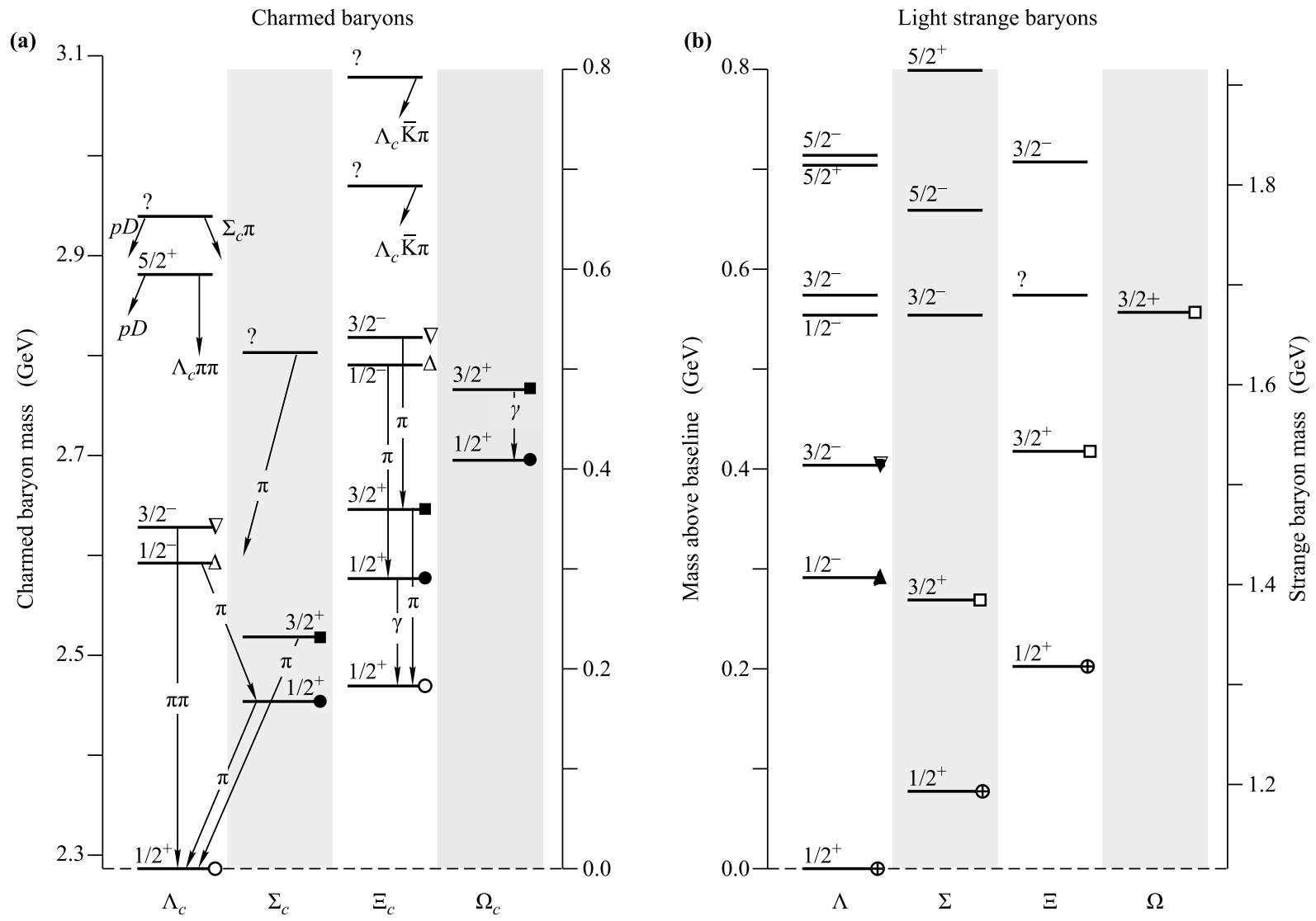

Fig. 2 Charmed baryons and their excitations [8]. 
Table 3 Mass spectra and widths (in units of MeV) of charmed baryons. Experimental values are taken from the Particle Data Group [8]. For the widths of the $\Sigma_{c}(2455)^{0 /++}$ and $\Sigma_{c}(2520)^{0 /++}$ baryons, we have taken into account the recent Belle measurement [11] for average. The width of $\Xi_{c}(2645)^{+}$is taken from Ref. [12]. For $\Xi_{c}(3055)^{0}$, we quote the preliminary result from Belle [13].

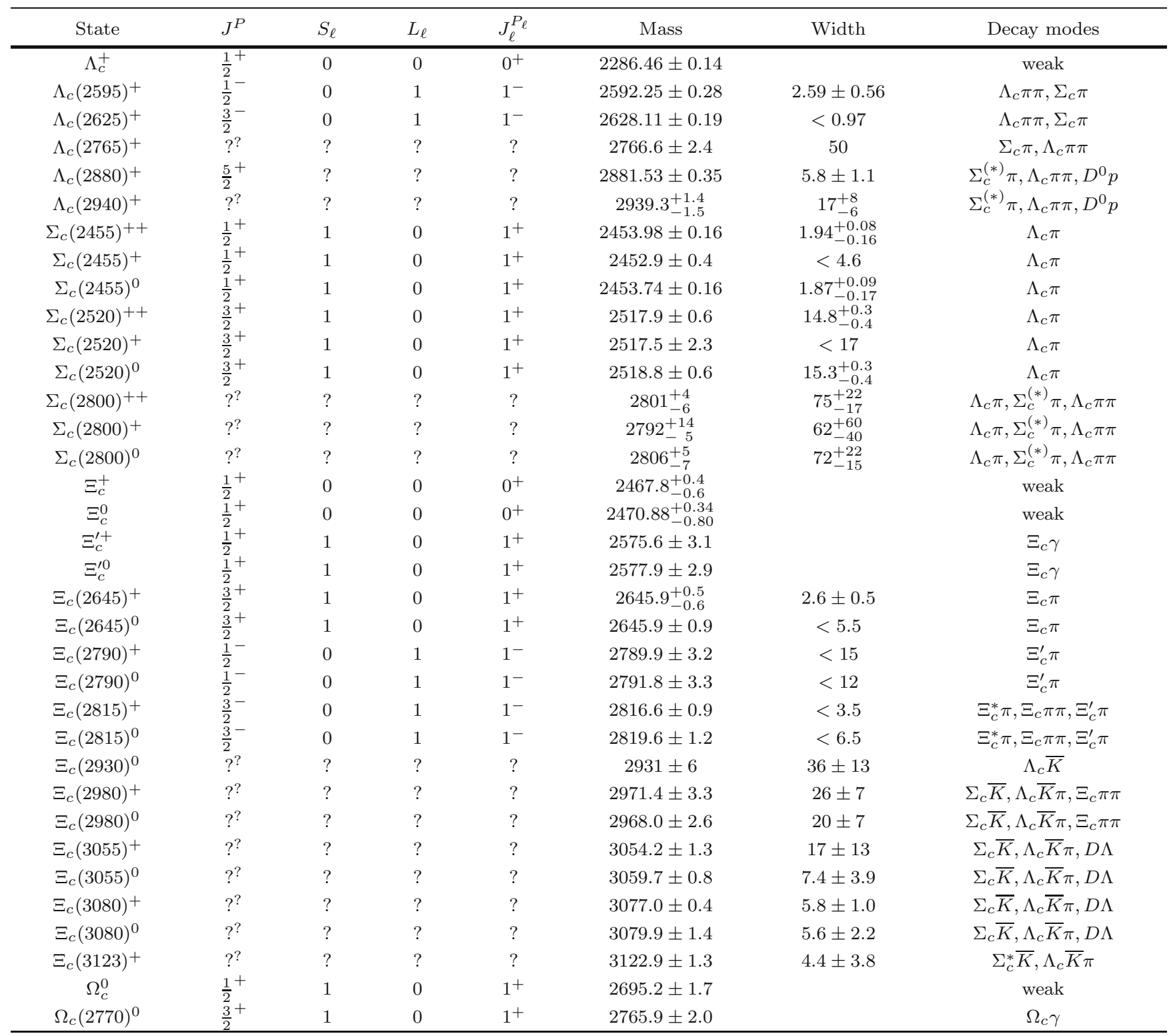

quantum numbers given in Table 3 has been measured. One has to rely on the quark model to determine the spin-parity assignments.

In the following, we discuss some of the excited charmed baryon states:

\subsection{1 $\Lambda_{c}$ states}

The lowest-lying $p$-wave $\Lambda_{c}$ states are $\tilde{\Lambda}_{c 0}\left(\frac{1}{2}^{-}\right)$, $\Lambda_{c 1}\left(\frac{1}{2}^{-}, \frac{3}{2}^{-}\right), \quad \tilde{\Lambda}_{c 1}\left(\frac{1}{2}^{-}, \frac{3}{2}^{-}\right)$and $\tilde{\Lambda}_{c 2}\left(\frac{3}{2}^{-}, \frac{5}{2}^{-}\right)$. The doublet $\Lambda_{c 1}\left(\frac{1}{2}^{-}, \frac{3}{2}^{-}\right)$is formed by $\Lambda_{c}(2595)^{+}$and $\Lambda_{c}(2625)^{+}$[14]. The allowed strong decays are
$\Lambda_{c 1}\left(1 / 2^{-}\right) \rightarrow\left[\Sigma_{c} \pi\right]_{S}, \quad\left[\Sigma_{c}^{*} \pi\right]_{D} \quad$ and $\quad \Lambda_{c 1}\left(3 / 2^{-}\right) \rightarrow$ $\left[\Sigma_{c} \pi\right]_{D},\left[\Sigma_{c}^{*} \pi\right]_{S, D},\left[\Lambda_{c} \pi \pi\right]_{P}$. This explains why the width of $\Lambda_{c}(2625)^{+}$is narrower than that of $\Lambda_{c}(2595)^{+}$. Because of isospin conservation in strong decays, $\Lambda_{c 1}^{+}$is not allowed to decay into $\Lambda_{c}^{+} \pi^{0}$.

$\Lambda_{c}(2765)^{+}$is a broad state first seen in $\Lambda_{c}^{+} \pi^{+} \pi^{-}$by CLEO [15]. However, it is still not known whether it is a $\Lambda_{c}^{+}$or a $\Sigma_{c}^{+}$and whether the width might be due to overlapping states. The Skyrme model [16] and the quark model $[17,18]$ suggest a $J^{P}=\frac{1}{2}^{+} \Lambda_{c}$ state with a mass of 2742 and $2775 \mathrm{MeV}$, respectively. Therefore, $\Lambda_{c}(2765)^{+}$ could be the first positive-parity excitation of $\Lambda_{c}$. In the 
diquark model, it has also been proposed to be either the first radial $(2 S)$ excitation of the $\Lambda_{c}$ with $J^{P}=\frac{1}{2}^{-}$ containing the light scalar diquark or the first orbital excitation $(1 P)$ of the $\Sigma_{c}$ with $J^{P}=\frac{3}{2}^{-}$containing the light axial vector diquark [19].

The state $\Lambda_{c}(2880)^{+}$, first observed by CLEO [15] in $\Lambda_{c}^{+} \pi^{+} \pi^{-}$, was also seen by BaBar in the $D^{0} p$ spectrum [20]. Belle studied the experimental constraint on the $J^{P}$ quantum numbers of $\Lambda_{c}(2880)^{+}$[21] and found that $J^{P}=\frac{5}{2}^{+}$is favored by the angular analysis of $\Lambda_{c}(2880)^{+} \rightarrow \Sigma_{c}^{0,++} \pi^{ \pm}$together with the ratio $\Sigma^{*} \pi / \Sigma \pi$, which was measured to be

$$
R \equiv \frac{\Gamma\left(\Lambda_{c}(2880) \rightarrow \Sigma_{c}^{*} \pi^{ \pm}\right)}{\Gamma\left(\Lambda_{c}(2880) \rightarrow \Sigma_{c} \pi^{ \pm}\right)}=\left(24.1 \pm 6.4_{-4.5}^{+1.1}\right) \% .
$$

In the quark model, the candidates for the parity-even spin- $\frac{5}{2}$ state are $\Lambda_{c 2}\left(\frac{5}{2}^{+}\right), \hat{\Lambda}_{c 2}\left(\frac{5}{2}^{+}\right), \tilde{\Lambda}_{c 2}^{1}\left(\frac{5}{2}^{+}\right), \tilde{\Lambda}_{c 2}^{2}\left(\frac{5}{2}^{+}\right)$, and $\tilde{\Lambda}_{c 3}^{2}\left(\frac{5}{2}^{+}\right)$(see Table 2). The first four candidates, with $J_{\ell}=2$, decay to $\Sigma_{c} \pi$ in an $F$ wave and to $\Sigma_{c}^{*} \pi$ in $F$ and $P$ waves. Neglecting the $P$-wave contribution for the moment,

$$
\begin{aligned}
& \frac{\Gamma\left(\Lambda_{c 2}\left(5 / 2^{+}\right) \rightarrow\left[\Sigma_{c}^{*} \pi\right]_{F}\right)}{\Gamma\left(\Lambda_{c 2}\left(5 / 2^{+}\right) \rightarrow\left[\Sigma_{c} \pi\right]_{F}\right)}=\frac{4}{5} \frac{p_{\pi}^{7}\left(\Lambda_{c}(2880) \rightarrow \Sigma_{c}^{*} \pi\right)}{p_{\pi}^{7}\left(\Lambda_{c}(2880) \rightarrow \Sigma_{c} \pi\right)} \\
& =\frac{4}{5} \times 0.29=0.23,
\end{aligned}
$$

where the factor of $4 / 5$ follows from heavy quark symmetry. At first glance, this appears to be in good agreement with experiment. However, the $\Sigma_{c}^{*} \pi$ channel is available via a $P$-wave and is enhanced by a factor of $1 / p_{\pi}^{4}$ relative to the $F$-wave one. However, heavy quark symmetry cannot be applied to calculate the contribution of the $\left[\Sigma_{c}^{*} \pi\right]_{F}$ channel to the ratio $R$, as the reduced matrix elements for the $P$-wave and $F$-wave modes differ. In this case, one has to rely on a phenomenological model to compute the ratio $R$. As for $\tilde{\Lambda}_{c 3}^{2}\left(\frac{5}{2}^{+}\right)$, it decays to $\Sigma_{c}^{*} \pi, \Sigma_{c} \pi$ and $\Lambda_{c} \pi$ all in $F$ waves. Further,

$$
\begin{aligned}
& \frac{\Gamma\left(\tilde{\Lambda}_{c 3}^{2}\left(5 / 2^{+}\right) \rightarrow\left[\Sigma_{c}^{*} \pi\right]_{F}\right)}{\Gamma\left(\tilde{\Lambda}_{c 3}^{2}\left(5 / 2^{+}\right) \rightarrow\left[\Sigma_{c} \pi\right]_{F}\right)}=\frac{5}{4} \frac{p_{\pi}^{7}\left(\Lambda_{c}(2880) \rightarrow \Sigma_{c}^{*} \pi\right)}{p_{\pi}^{7}\left(\Lambda_{c}(2880) \rightarrow \Sigma_{c} \pi\right)} \\
& =\frac{5}{4} \times 0.29=0.36 .
\end{aligned}
$$

Although this deviates from the experimental measurement $(2.1)$ by $1 \sigma$, it is a robust prediction. This has motivated us to conjecture that the first positive-parity excited charmed baryon $\Lambda_{c}(2880)^{+}$could be an admixture of $\Lambda_{c 2}\left(\frac{5}{2}^{+}\right), \hat{\Lambda}_{c 2}\left(\frac{5}{2}^{+}\right)$and $\tilde{\Lambda}_{c 3}^{2}\left(\frac{5}{2}^{+}\right)[10]$.
It is worth mentioning that the Peking group [22] has studied the strong decays of charmed baryons on the basis of the so-called ${ }^{3} P_{0}$ recombination model. For the $\Lambda_{c}(2880)$, the Peking group found that (i) $\Lambda_{c}(2880)$ cannot be a radial excitation, as its decay into $D^{0} p$ is prohibited in the ${ }^{3} P_{0}$ model if $\Lambda_{c}(2880)$ is the first radial excitation of $\Lambda_{c}$, and (ii) the states $\Lambda_{c 2}\left(\frac{5}{2}^{+}\right), \tilde{\Lambda}_{c 2}^{1}\left(\frac{5}{2}^{+}\right)$ and $\hat{\Lambda}_{c 2}\left(\frac{5}{2}^{+}\right)$are excluded, as they do not decay to $D^{0} p$ according to the ${ }^{3} P_{0}$ model. Moreover, the predicted ratios $\Sigma_{c}^{*} \pi / \Sigma_{c} \pi$ are either too large or too small compared to experiment, for example,

$$
\begin{aligned}
& \frac{\Gamma\left(\Lambda_{c 2}\left(5 / 2^{+}\right) \rightarrow \Sigma_{c}^{*} \pi\right)}{\Gamma\left(\Lambda_{c 2}\left(5 / 2^{+}\right) \rightarrow \Sigma_{c} \pi\right)}=89, \\
& \frac{\Gamma\left(\hat{\Lambda}_{c 2}\left(5 / 2^{+}\right) \rightarrow \Sigma_{c}^{*} \pi\right)}{\Gamma\left(\hat{\Lambda}_{c 2}\left(5 / 2^{+}\right) \rightarrow \Sigma_{c} \pi\right)}=0.75 .
\end{aligned}
$$

Both symmetric states $\Lambda_{c 2}$ and $\hat{\Lambda}_{c 2}$, are thus ruled out. Hence, it appears that $\tilde{\Lambda}_{c 3}^{2}\left(\frac{5}{2}^{+}\right)$dictates the inner structure of $\Lambda_{c}(2880) .{ }^{1)}$ However, there are several problems with this assignment: (i) the quark model indicates a $\Lambda_{c 2}\left(\frac{5}{2}^{+}\right)$state around $2910 \mathrm{MeV}$, which is close to the mass of $\Lambda_{c}(2880)$, whereas the mass of $\tilde{\Lambda}_{c 3}^{2}\left(\frac{5}{2}^{+}\right)$is even higher $[17,18]$, (ii) $\tilde{\Lambda}_{c 3}^{2}\left(\frac{5}{2}^{+}\right)$can decay to an $F$-wave $\Lambda_{c} \pi$, and this has not been seen by BaBar and Belle, and (iii) the calculated width, $28.8 \mathrm{MeV}$, is too large compared to the measured one, $5.8 \pm 1.1 \mathrm{MeV}$. One may argue that the ${ }^{3} P_{0}$ model's prediction can easily differ from the experimental measurement by a factor of $2-3$ owing to its inherent uncertainties [22].

Interestingly, the quantum numbers $J^{P}=\frac{5}{2}^{+}$for the $\Lambda_{c}(2880)$ were correctly predicted on the basis of the diquark concept in Refs. [24, 25] before the Belle experiment.

The highest state $\Lambda_{c}(2940)^{+}$was first discovered by BaBar in the $D^{0} p$ decay mode [20] and confirmed by Belle in the decays $\Sigma_{c}^{0} \pi^{+}, \Sigma_{c}^{++} \pi^{-}$, which subsequently decay into $\Lambda_{c}^{+} \pi^{+} \pi^{-}$[21]. Its spin-parity assignment is quite diverse. For example, it has been argued that $\Lambda_{c}(2940)^{+}$is the radial excitation of $\Lambda_{c}(2595)$ with $J^{P}=$ $\frac{1}{2}^{-}$, but the predicted mass is too large by the order of $40 \mathrm{MeV}$. Alternatively, it could be the first radial excitation of $\Sigma_{c}\left(\operatorname{not} \Lambda_{c} !\right)$ with $J^{P}=3 / 2^{+}$[26]. The latter assignment has the advantage that the predicted mass is in better agreement with experiment. Because the mass of $\Lambda_{c}(2940)^{+}$is barely below the threshold of $D^{* 0} p$, this observation motivated the authors of Ref. [27] to suggest

\footnotetext{
1) It has been argued in Ref. [23] that in the chiral quark model $\Lambda_{c}(2880)$ favors to be the state $\left|\Lambda_{c}{ }^{2 S+1} L_{\sigma} J^{P}\right\rangle=\left|\Lambda_{c}{ }^{2} D_{\lambda \lambda} \frac{3}{2}^{+}\right\rangle$with $L_{\rho}=0$ and $L_{\lambda}=2$ rather than $\left|\Lambda_{c}^{2} D_{A} \frac{5}{2}^{+}\right\rangle$with $L_{\rho}=L_{\lambda}=1$ as the latter cannot decay into $D^{0} p$. However, this is not our case as $\tilde{\Lambda}_{c 3}^{2}\left(\frac{5}{2}^{+}\right)$does decay to $D^{0} p$ and can reproduce the measured value of $R$.
} 
an exotic molecular state of $D^{* 0}$ and $p$ with a binding energy of the order of $6 \mathrm{MeV}$ and $J^{P}=\frac{1}{2}^{-}$for $\Lambda_{c}(2940)^{+}$. The quark potential model predicts a $\frac{5}{2}^{-} \Lambda_{c}$ state at 2900 $\mathrm{MeV}$ and $\frac{3}{2}^{+} \Lambda_{c}$ state at $2910 \mathrm{MeV}[17,18]$. A similar result of $2906 \mathrm{MeV}$ for $\frac{3}{2}^{+} \Lambda_{c}$ was also obtained in the relativistic quark model [28].

\subsection{2 $\Sigma_{c}$ states}

The highest isotriplet charmed baryons, $\Sigma_{c}(2800)^{++,+, 0}$, which decay to $\Lambda_{c}^{+} \pi$, were first measured by Belle [29] with widths of the order of $70 \mathrm{MeV}$. The possible quark states are $\Sigma_{c 0}\left(\frac{1}{2}^{-}\right), \Sigma_{c 1}\left(\frac{1}{2}^{-}, \frac{3}{2}^{-}\right), \tilde{\Sigma}_{c 1}\left(\frac{1}{2}^{-}, \frac{3}{2}^{-}\right)$, and $\Sigma_{c 2}\left(\frac{3}{2}^{-}, \frac{5}{2}^{-}\right)$. The states $\Sigma_{c 1}$ and $\tilde{\Sigma}_{c 1}$ are ruled out because their decays to $\Lambda_{c}^{+} \pi$ are prohibited in the heavy quark limit. Now the $\Sigma_{c 2}\left(\frac{3}{2}^{-}, \frac{5}{2}^{-}\right)$baryon decays primarily into the $\Lambda_{c} \pi$ system in a $D$-wave, whereas $\Sigma_{c 0}\left(\frac{1}{2}^{-}\right)$ decays into $\Lambda_{c} \pi$ in an $S$-wave. Because heavy hadron chiral perturbation theory (HHChPT) implies a very broad $\Sigma_{c 0}$ with a width of the order of $885 \mathrm{MeV}$ (see Section 3.2 below), this $p$-wave state is also excluded. Therefore, $\Sigma_{c}(2800)^{++,+, 0}$ are likely to be either $\Sigma_{c 2}\left(\frac{3}{2}{ }^{-}\right)$or $\Sigma_{c 2}\left(\frac{5}{2}^{-}\right)$or a mixture of the two. In the quark-diquark model [26], both of them have very close masses compatible with experiment. Given that for light strange baryons, the first orbital excitation of the $\Sigma$ also has the quantum numbers $J^{P}=3 / 2^{-}$(see Fig. 2), we will advocate a $\Sigma_{c 2}\left(3 / 2^{-}\right)$state for $\Sigma_{c}(2800)$.

\subsection{3 $\Xi_{c}$ states}

The states $\Xi_{c}(2790)$ and $\Xi_{c}(2815)$ form the doublet $\Xi_{c 1}\left(\frac{1}{2}^{-}, \frac{3}{2}^{-}\right)$. Because the diquark transition $1^{-} \rightarrow 0^{+}+\pi$ is prohibited, $\Xi_{c 1}\left(\frac{1}{2}^{-}, \frac{3}{2}^{-}\right)$cannot decay to $\Xi_{c} \pi$. The dominant decay modes are $\left[\Xi_{c}^{\prime} \pi\right]_{S}$ for $\Xi_{c 1}\left(\frac{1}{2}^{-}\right)$and $\left[\Xi_{c}^{*} \pi\right]_{S}$ for $\Xi_{c 1}\left(\frac{3}{2}^{-}\right)$.

Many excited charmed baryon states $\Xi_{c}(2980)$, $\Xi_{c}(3055), \Xi_{c}(3080)$, and $\Xi_{c}(3123)$ have been seen at $B$ factories $[12,30,31]$. Another state $\Xi_{c}(2930)^{0}$, which is omitted from the PDG summary table, has been seen only by BaBar in the $\Lambda_{c}^{+} K^{-}$mass projection of $B^{-} \rightarrow \Lambda_{c}^{+} \bar{\Lambda}_{c}^{-} K^{-}$[33]. However, as we shall see below, it may form a sextet with $\Sigma_{c}(2800)$ and $\Omega_{c}(3050)$. The states $\Xi_{c}(2980), \Xi_{c}(3055), \Xi_{c}(3080)$, and $\Xi_{c}(3123)$ could be the first positive-parity excitations of the $\Xi_{c}$. The study of Regge phenomenology is very useful for the $J^{P}$ assignment of charmed baryons [26, 34]. The Regge analysis suggests that $J^{P}=3 / 2^{+}$for $\Xi_{c}(3055)$ and $5 / 2^{+}$for $\Xi_{c}(3080)$ [26]. From Table 5 below, we shall see that $\Xi_{c}(3080)$ and $\Lambda_{c}(2880)$ form a nice $J^{P}=5 / 2^{+}$ antitriplet.

In the relativistic quark-diquark model [26], $\Xi_{c}(2980)$ is a sextet $J^{P}=\frac{1}{2}^{+}$state. According to Table 2, possible candidates are $\Xi_{c 1}^{\prime}\left(\frac{1}{2}^{+}\right), \hat{\Xi}_{c 1}^{\prime}\left(\frac{1}{2}^{+}\right), \tilde{\Xi}_{c 0}^{\prime}\left(\frac{1}{2}^{+}\right)$, and $\tilde{\Xi}_{c 1}^{\prime}\left(\frac{1}{2}^{+}\right)$. As pointed out in Ref. [32], strong decays of these four states, which were studied in Ref. [22] using the ${ }^{3} P_{0}$ model show that $\tilde{\Xi}_{c 1}^{\prime}\left(\frac{1}{2}^{+}\right)$does not decay to $\Xi_{c} \pi$ and $\Lambda_{c} \bar{K}$, and has a width of $28 \mathrm{MeV}$, consistent with experiment. Therefore, the favored candidate for $\Xi_{c}(2980)$ is $\tilde{\Xi}_{c 1}^{\prime}\left(\frac{1}{2}^{+}\right)$, which has $J_{\ell}=L_{\ell}=1$.

The possible quark states for the $J^{P}=\frac{5}{2}^{+} \Xi_{c}(3080)$ baryon in an antitriplet are $\Xi_{c 2}\left(\frac{5}{2}^{+}\right), \hat{\Xi}_{c 2}\left(\frac{5}{2}^{+}\right), \tilde{\Xi}_{c 2}^{1}\left(\frac{5}{2}^{+}\right)$, $\tilde{\Xi}_{c 2}^{2}\left(\frac{5}{2}^{+}\right)$, and $\tilde{\Xi}_{c 3}^{2}\left(\frac{5}{2}^{+}\right)$(see Table 2). Because $\Xi_{c}(3080)$ is above the $D \Lambda$ threshold, the two-body mode $D \Lambda$ should exist although it has not been searched for in the $D \Lambda$ spectrum. Recall that the neutral $\Xi_{c}(3055)^{0}$ was observed recently by Belle in the $D^{0} \Lambda$ spectrum [13]. According to the ${ }^{3} P_{0}$ model, the first four states are excluded as they do not decay into $D \Lambda$ [22]. The only remaining possibility is $\tilde{\Xi}_{c 3}^{2}\left(\frac{5}{2}^{+}\right)$. This is the analog of $\tilde{\Lambda}_{c 3}^{2}\left(\frac{5}{2}^{+}\right)$for $\Lambda_{c}(2880)$. Nevertheless, the identification of $\tilde{\Xi}_{c 3}^{2}\left(\frac{5}{2}^{+}\right)$with $\Xi_{c}(3080)$ encounters two potential problems: (i) its width is dominated by the $\Xi_{c} \pi$ and $\Lambda_{c}^{+} \bar{K}$ modes, which have not been seen experimentally, and (ii) the predicted width of the order of $47 \mathrm{MeV}$ [22] is too large compared to the measured one, which is of the order of $5.7 \mathrm{MeV}$.

\subsection{4 $\Omega_{c}$ states}

Only two ground states have been observed thus far: $1 / 2^{+} \Omega_{c}^{0}$ and $3 / 2^{+} \Omega_{c}(2770)^{0}$. The latter was seen by BaBar in the electromagnetic decay $\Omega_{c}(2770) \rightarrow \Omega_{c} \gamma$ [39].

\section{Molecular picture}

Because $\Lambda_{c}(2940)^{+}$and $\Sigma_{c}(2800)$ are barely below the $D^{* 0} p$ and $D N$ thresholds, respectively, it is tempting to conjecture an exotic molecular structure of $D^{* 0}$ and $p$ for the former and a molecular state of $D N$ for the latter [27, 40-44]. Likewise, $\Xi_{c}(2980)$ could be a molecular state of $D \Lambda$.

A coupled-channel calculation of the baryon-meson $N D$ system has been performed to look for an isospinspin channel that is attractive enough to form a molecular state $[42,45]$. (I) $J^{P}=(0) \frac{1}{2}^{-}$was found to be the most attractive one, followed by $(I) J^{P}=(1) \frac{3}{2}^{-}$. This suggests that $\Sigma_{c}(2800)$ might be an $s$-wave $D N$ molecular state with $(I) J^{P}=(0) \frac{1}{2}^{-}$and $\Lambda_{c}(2940)$ an $s$-wave $D^{*} N$ molecular state with $(I) J^{P}=(1) \frac{3}{2}^{-}$(see Fig. 3 
of [45]). Another possibility is a $D N$ molecular state with $(I) J^{P}=(1) \frac{3}{2}^{-}$for $\Sigma_{c}(2800)$ and a $D^{*} N$ state with $(I) J^{P}=(0) \frac{1}{2}^{-}$for $\Lambda_{c}(2940)$. Because $\Sigma_{c}(2800)$ has isospin 1, and moreover, as we have noted in passing, $\Sigma_{c}(2800)$ will be too broad if it is assigned to $J^{P}=1 / 2^{-}$, we conclude that the second possibility is preferable (see also [44]).

The possible spin-parity quantum numbers of the higher excited charmed baryon resonances that have been suggested in the literature are partially summarized in Table 4. Some of the predictions are already ruled out by experiment. For example, $\Lambda_{c}(2880)$ has $J^{P}=\frac{5}{2}^{+}$, as seen by Belle. More experimental studies are certainly to pin down the quantum numbers.

Charmed baryon spectroscopy has been studied extensively in various models. It appears that the spectroscopy is well described by the heavy quark-light diquark picture elaborated by Ebert, Faustov, and Galkin (EFG) [26] (see also Ref. [35]). As noted in passing, the quantum numbers $J^{P}=\frac{5}{2}^{+}$of $\Lambda_{c}(2880)$ were correctly predicted in a model based on the diquark idea before the Belle experiment [24, 25]. Moreover, EFG have shown that all the available experimental data on heavy baryons fit the linear Regge trajectories nicely, namely, the trajectories in the $\left(J, M^{2}\right)$ and $\left(n_{r}, M^{2}\right)$ planes for orbitally and radially excited heavy baryons, respectively,

$$
J=\alpha M^{2}+\alpha_{0}, \quad n_{r}=\beta M^{2}+\beta_{0},
$$

where $n_{r}$ is the radial excitation quantum number, $\alpha$ and $\beta$ are the slopes, and $\alpha_{0}$ and $\beta_{0}$ are intercepts. The linearity, parallelism, and equidistance of the Regge trajectories were verified. The predictions of the spin-parity quantum numbers of charmed baryons and their masses in Ref. [26] can be regarded as a theoretical benchmark (see Fig. 3).

$\underline{\text { Antitriplet and sextet states }}$

The antitriplet and sextet states of charmed baryons are listed in Table 5. To date, the following states are established: the $J^{P}=\frac{1}{2}^{+}, \frac{1}{2}^{-}$, and $\frac{3}{2}^{-} \overline{\mathbf{3}}$ states, $\left(\Lambda_{c}^{+}, \Xi_{c}^{+}, \Xi_{c}^{0}\right),\left(\Lambda_{c}(2595)^{+}, \Xi_{c}(2790)^{+}, \Xi_{c}(2790)^{0}\right)$, and $\left(\Lambda_{c}(2625)^{+}, \Xi_{c}(2815)^{+}, \Xi_{c}(2815)^{0}\right)$, respectively, and the $J^{P}=\frac{1}{2}^{+}$and $\frac{3}{2}^{+} \mathbf{6}$ states, $\left(\Omega_{c}, \Sigma_{c}, \Xi_{c}^{\prime}\right)$ and $\left(\Omega_{c}^{*}, \Sigma_{c}^{*}, \Xi_{c}^{\prime *}\right)$, respectively. The mass difference $m_{\Xi_{c}}-m_{\Lambda_{c}}$ in the antitriplet states clearly lies between 180 and $200 \mathrm{MeV}$. We note in passing that $\Xi_{c}(3080)$ should carry the quantum numbers $J^{P}=5 / 2^{+}$. From Table 5 , we see that $\Xi_{c}(3080)$ and $\Lambda_{c}(2880)$ form a nice $J^{P}=5 / 2^{+}$antitriplet as the mass difference between $\Xi_{c}(3080)$ and $\Lambda_{c}(2880)$ is consistent with that observed in other antitriplets. Likewise, the mass differences in the $J^{P}=3 / 2^{-}$sextet $\left(\Omega_{c}(3050), \Xi_{c}^{\prime}(2930), \Sigma_{c}(2800)\right)$ predicted by the quarkdiquark model are consistent with that measured in $J^{P}=1 / 2^{+}$and $3 / 2^{+}$sextets. Note that there is no $J^{P}=\frac{1}{2}^{-}$sextet as the $\Sigma_{c}(2800)$ with these spin-parity quantum numbers will be too broad to be observed.

On the basis of the QCD sum rules, many charmed baryon multiplets classified according to $\left[\mathbf{6}_{F}\left(\right.\right.$ or $\left.\overline{\mathbf{3}}_{F}\right)$, $\left.\left.J_{\ell}, S_{\ell}, \rho / \lambda\right)\right]$ were recently studied in Ref. [36]. Three sex-

\section{Charmed baryon spectra}

\begin{tabular}{|c|c|c|c|}
\hline & & $\begin{array}{l}\Xi_{c}^{\prime}(3123) 7 / 2^{+}(1 \mathrm{D}) \\
\underline{\Xi}_{c}(3080) 5 / 2^{+}(1 \mathrm{D}) \\
\Xi_{c}(3055) 3 / 2^{+} \\
\underline{G}^{+}(1 \mathrm{D})\end{array}$ & \\
\hline & & ${\underline{\underline{\Xi_{c}^{\prime}}}}_{(2980)} 1 / 2^{+}(2 S)$ & \\
\hline$\Lambda_{c}(2940) ? ?$ & & ${\underline{\underline{\Xi_{c}^{\prime}}}}_{c}(2930) 3 / 2^{-}(1 \mathrm{P})$ & \\
\hline$\underline{\Lambda}_{c}(2880) 5 / 2^{+}$(1D) & & & \\
\hline & $\underline{\Sigma_{c}(2800) 3 / 2^{-}}(1 \mathrm{P})$ & $\begin{array}{lll}\Xi_{c}(2815) & 3 / 2 & (1 \mathrm{P}) \\
\Xi_{c}(2790) & 1 / 2 & (1 \mathrm{P})\end{array}$ & \\
\hline$\frac{\Lambda_{c}(2765) 1 / 2^{+}}{\Sigma_{c}(2765) 3 / 2^{-}}(1 \mathrm{P})$ & & & $\underline{\Omega_{c}(2770) 3 / 2^{+}}$ \\
\hline$\Lambda_{c}(2625) 3 / 2{ }^{-}(1 \mathrm{P})$ & & $\underline{\underline{\Xi}}_{c}^{\prime}(2645) 3 / 2^{+}$ & $\frac{3 x_{c}(2050)}{1 / 2}$ \\
\hline$\underline{\Lambda}_{c}(2595) 1 / 2-(1 \mathrm{P})$ & & ${\underline{\underline{\Xi_{d}^{\prime}}}}_{d}(2575) 1 / 2^{+}$ & \\
\hline & $\underline{\Sigma_{c}(2520)} 3 / 2^{+}$ & & \\
\hline & $\Sigma_{c}(2455) 1 / 2^{+}$ & ${\underline{\Xi_{c}}}(2470) 1 / 2^{+}$ & \\
\hline$\underline{\Lambda_{c}(2287) 1 / 2^{+}}$ & & & \\
\hline$\Lambda_{\mathrm{c}}$ & $\Sigma_{\mathrm{c}}$ & $\Xi_{\mathrm{c}}$ & $\Omega_{c}$ \\
\hline
\end{tabular}

Fig. 3 Singly charmed baryon states where the spin-parity quantum numbers in red are taken from Ref. [26].

Table 4 Possible spin-parity quantum numbers for excited charmed baryon resonances.

\begin{tabular}{|c|c|c|c|c|c|c|c|c|c|}
\hline & $\Lambda_{c}(2765)$ & $\Lambda_{c}(2880)$ & $\Lambda_{c}(2940)$ & $\Sigma_{c}(2800)$ & $\Xi_{c}(2930)$ & $\Xi_{c}(2980)$ & $\Xi_{c}(3055)$ & $\Xi_{c}(3080)$ & $\Xi_{c}(3123)$ \\
\hline Capstick et al. $[17,18]$ & $\frac{1}{2}^{+}$ & & $\frac{3}{2}^{+}, \frac{5}{2}^{-}$ & $\frac{3}{2}^{-}, \frac{5}{2}^{-}$ & & & & & \\
\hline B. Chen et al. [35] & $\frac{1}{2}^{+}(2 S)$ & $\frac{5}{2}^{+}(1 D)$ & $\frac{1}{2}-(2 P)$ & & & $\frac{1}{2}^{+}(2 S)$ & $\frac{3}{2}^{+}(1 D)$ & $\frac{5}{2}^{+}(1 D)$ & $\frac{1}{2}^{-}(2 P)$ \\
\hline H. Chen et al. [36] & & & $\frac{1}{2}^{+}, \frac{1}{2}^{-}$ & $\frac{1}{2}^{-}, \frac{3}{2}^{-}$ & & $\frac{1}{2}-, \frac{3}{2}^{-}$ & & $\frac{5}{2}^{-}$ & \\
\hline Cheng et al. [10] & & & & & & & & & \\
\hline Ebert et al. [26] & $\frac{1}{2}^{+}(2 S)$ & $\frac{5}{2}^{+}(1 D)$ & $\frac{1}{2}^{-}(2 P), \frac{3}{2}^{+}(2 S)$ & $\frac{1}{2}^{-}, \frac{3}{2}^{-}(1 P)$ & $\frac{1}{2}^{-}, \frac{3}{2}^{-}, \frac{5}{2}^{-}$ & $\frac{1}{2}^{+}(2 S)$ & $\frac{3}{2}^{+}(1 D)$ & $\frac{5}{2}^{+}(1 D)$ & $\frac{7}{2}^{+}(1 D)$ \\
\hline Garcilazo et al. [28] & $\frac{1}{2}^{+}$ & $\frac{1}{2}^{-}, \frac{3}{2}^{-}$ & $\frac{3}{2}^{+}$ & $\frac{1}{2}^{-}, \frac{3}{2}^{-}$ & & & & & \\
\hline Wilczek et al. $[24,25]$ & & $\frac{5}{2}^{+}$ & & & & & & & \\
\hline Zhong et al. [23] & $\frac{1}{2}^{-}(1 P)$ & $\frac{3}{2}^{+}(1 D)$ & $\frac{5}{2}^{+}(1 D)$ & $\frac{1}{2}^{-}, \frac{5}{2}^{-}(1 P)$ & & & & & \\
\hline
\end{tabular}


Table 5 Antitriplet and sextet states of charmed baryons. The $J^{P}$ quantum numbers of $\Xi_{c}(3080), \Xi_{c}^{\prime}(2930), \Sigma_{c}(2800)$ are not yet established and the $\Omega_{c}\left(3 / 2^{-}\right)$state has not been observed. Mass differences $\Delta m_{\Xi_{c} \Lambda_{c}} \equiv m_{\Xi_{c}}-m_{\Lambda_{c}}, \Delta m_{\Xi_{c}^{\prime} \Sigma_{c}} \equiv m_{\Xi_{c}^{\prime}}-m_{\Sigma_{c}}, \Delta m_{\Omega_{c} \Xi_{c}^{\prime}} \equiv$ $m_{\Omega_{c}}-m_{\Xi_{c}^{\prime}}$ are in units of $\mathrm{MeV}$.

\begin{tabular}{ccccc}
\hline & $J^{P}$ & States & Mass difference & Status \\
\hline$\overline{\mathbf{3}}$ & $1 / 2^{+}$ & $\Lambda_{c}(2287)^{+}, \Xi_{c}(2470)^{+}, \Xi_{c}(2470)^{0}$ & $\Delta m_{\Xi_{c} \Lambda_{c}}=183$ & estab \\
& $1 / 2^{-}$ & $\Lambda_{c}(2595)^{+}, \Xi_{c}(2790)^{+}, \Xi_{c}(2790)^{0}$ & $\Delta m_{\Xi_{c} \Lambda_{c}}=198$ & estab \\
& $3 / 2^{-}$ & $\Lambda_{c}(2625)^{+}, \Xi_{c}(2815)^{+}, \Xi_{c}(2815)^{0}$ & $\Delta \Xi_{\Xi_{c} \Lambda_{c}}=190$ & estab \\
& $5 / 2^{+}$ & $\Lambda_{c}(2880)^{+}, \Xi_{c}(3080)^{+}, \Xi_{c}(3080)^{0}$ & $\Delta m_{\Xi_{c} \Lambda_{c}}=196$ & {$[26]$} \\
\hline $\mathbf{6}$ & $1 / 2^{+}$ & $\Omega_{c}(2695)^{0}, \Xi_{c}^{\prime}(2575)^{+, 0}, \Sigma_{c}(2455)^{++,+, 0}$ & $\Delta m_{\Xi_{c}^{\prime} \Sigma_{c}}=124, \Delta m_{\Omega_{c} \Xi_{c}^{\prime}}=119$ & estab \\
& $3 / 2^{+}$ & $\Omega_{c}(2770)^{0}, \Xi_{c}^{\prime}(2645)^{+, 0}, \Sigma_{c}(2520)^{++,+, 0}$ & $\Delta m_{\Xi_{c}^{\prime} \Sigma_{c}}=128, \Delta m_{\Omega_{c} \Xi_{c}^{\prime}}=120$ & estab \\
& $3 / 2^{-}$ & $\Omega_{c}(3050)^{0}, \Xi_{c}^{\prime}(2930)^{+, 0}, \Sigma_{c}(2800)^{++,+, 0}$ & $\Delta m_{\Xi_{c}^{\prime} \Sigma_{c}}=131, \Delta m_{\Omega_{c} \Xi_{c}^{\prime}}=119$ & {$[26]$} \\
\hline
\end{tabular}

tets were proposed in this work: $\left(\Omega_{c}(3250), \Xi_{c}^{\prime}(2980)\right.$, $\left.\Sigma_{c}(2800)\right)$ for $J^{P}=1 / 2^{-}, 3 / 2^{-}$and $\left(\Omega_{c}(3320), \Xi_{c}^{\prime}(3080)\right.$, $\left.\Sigma_{c}(2890)\right)$ for $J^{P}=5 / 2^{-}$. Note that $\Xi_{c}^{\prime}(2980)$ and $\Xi_{c}^{\prime}(3080)$ were treated as $p$-wave baryons rather than the first positive-parity excitations, as we have discussed before. The results for the multiplet $\left[\mathbf{6}_{F}, 1,0, \rho\right]$ led the authors of Ref. [36] to suggest that there are two $\Sigma_{c}(2800)$, $\Xi_{c}^{\prime}(2980)$, and $\Omega_{c}(3250)$ states with $J^{P}=1 / 2^{-}$and $J^{P}=3 / 2^{-}$. The mass splittings are $14 \pm 7,12 \pm 7$, and $10 \pm 6 \mathrm{MeV}$, respectively. The predicted mass of $\Omega_{c}\left(1 / 2^{-}, 3 / 2^{-}\right)$is around $3250 \pm 200 \mathrm{MeV}$. Using the central value of the predicted masses to label the states in the multiplet $\left[\mathbf{6}_{F}, 1,0, \rho\right]$ (see Table I of Ref. [36]), one obtains $\left(\Omega_{c}(3250), \Xi_{c}^{\prime}(2960), \Sigma_{c}(2730)\right)$ for $J^{P}=1 / 2^{-}$ and $\left(\Omega_{c}(3260), \Xi_{c}^{\prime}(2980), \Sigma_{c}(2750)\right)$ for $J^{P}=3 / 2^{-}$. One can check that $\Delta m_{\Xi_{c}^{\prime} \Sigma_{c}}=230 \pm 234 \mathrm{MeV}$, and $\Delta m_{\Omega_{c} \Xi_{c}^{\prime}}$ is of the order of $285 \pm 250 \mathrm{MeV}$. Owing to the large theoretical uncertainties in the masses, it is not clear whether the QCD sum rule calculations are compatible with the mass differences measured in the $J^{P}=1 / 2^{+}$and $3 / 2^{+}$ sextets. In any event, it will be interesting to test these two different model predictions for $J^{P}=3 / 2^{-}$and $1 / 2^{-}$ sextets in the future.

\subsection{Doubly charmed baryons}

Evidence of doubly charmed baryon states has been reported by SELEX in $\Xi_{c c}(3520)^{+} \rightarrow \Lambda_{c}^{+} K^{-} \pi^{+}$[46]. Further observation of $\Xi_{c c}^{+} \rightarrow p D^{+} K^{-}$was also announced by SELEX [47]. However, none of the doubly charmed states discovered by SELEX has been confirmed by FOCUS [48], BaBar [49], Belle [12] and LHCb [50], although $10^{6} \Lambda_{c}$ events are produced in $B$ factories, for example, versus $1630 \Lambda_{c}$ events observed at SELEX.

The doubly charmed baryons $\Xi_{c c}^{(*)++}, \Xi_{c c}^{(*)+}, \Omega_{c c}^{(*)+}$ with the quark contents ccu, ccd,ccs form an $\mathrm{SU}(3)$ triplet. They have been studied extensively using many different approaches: the quark model, light quarkheavy diquark model, QCD sum rules, and lattice simu- lation. The predicted doubly charmed baryon masses calculated in various models are tabulated in Refs. [51, 52]. For recent QCD sum rule calculations, see e.g. [53-55]. Chiral corrections to the masses of doubly heavy baryons up to $\mathrm{N}^{3} \mathrm{LO}$ were presented in Ref. [56].

Figure 4 shows the results of recent lattice studies of doubly and triply charmed baryon spectra by different groups: RQCD [57], HSC [58], Brown et al. [59], ETMC [60], ILGTI [61], PACS-CS [62], Durr et al. [63], Briceno et al. [64], Liu et al. [65], and $\mathrm{Na}$ et al. [66]. A new lattice calculation of $\Omega_{c c}^{(*)}$ and $\Omega_{c c c}$ was available in Ref. [67]. The various lattice results are consistent with each other and they fall into the ranges

$$
\begin{aligned}
& M\left(\Xi_{c c}\right)=3.54-3.68 \mathrm{GeV}, \\
& M\left(\Xi_{c c}^{*}\right)=3.61-3.72 \mathrm{GeV}, \\
& M\left(\Omega_{c c}\right)=3.57-3.76 \mathrm{GeV}, \\
& M\left(\Omega_{c c}^{*}\right)=3.68-3.85 \mathrm{GeV},
\end{aligned}
$$

and

$$
M\left(\Omega_{c c c}\right)=4.70-4.84 \mathrm{MeV} .
$$

Although lattice studies suggest that the mass of the low-lying $\Xi_{c c}$ exceeds $3519 \mathrm{MeV}$, it is interesting to note that the authors of [51] calculated the masses of doubly and triply charmed baryons on the basis of the Regge phenomenology and found $M\left(\Xi_{c c}^{+}\right)=3520.2_{-39.8}^{+40.6} \mathrm{MeV}$, in good agreement with SELEX.

\section{$3 \quad$ Strong decays}

Owing to the rich mass spectrum and relatively narrow widths of the excited states, the charmed baryon system offers an excellent ground for testing the ideas and predictions of heavy quark symmetry and light flavor $\mathrm{SU}(3)$ symmetry. The pseudoscalar mesons involved in the strong decays of charmed baryons such as $\Sigma_{c} \rightarrow \Lambda_{c} \pi$ are soft. Therefore, heavy quark symmetry of the heavy 


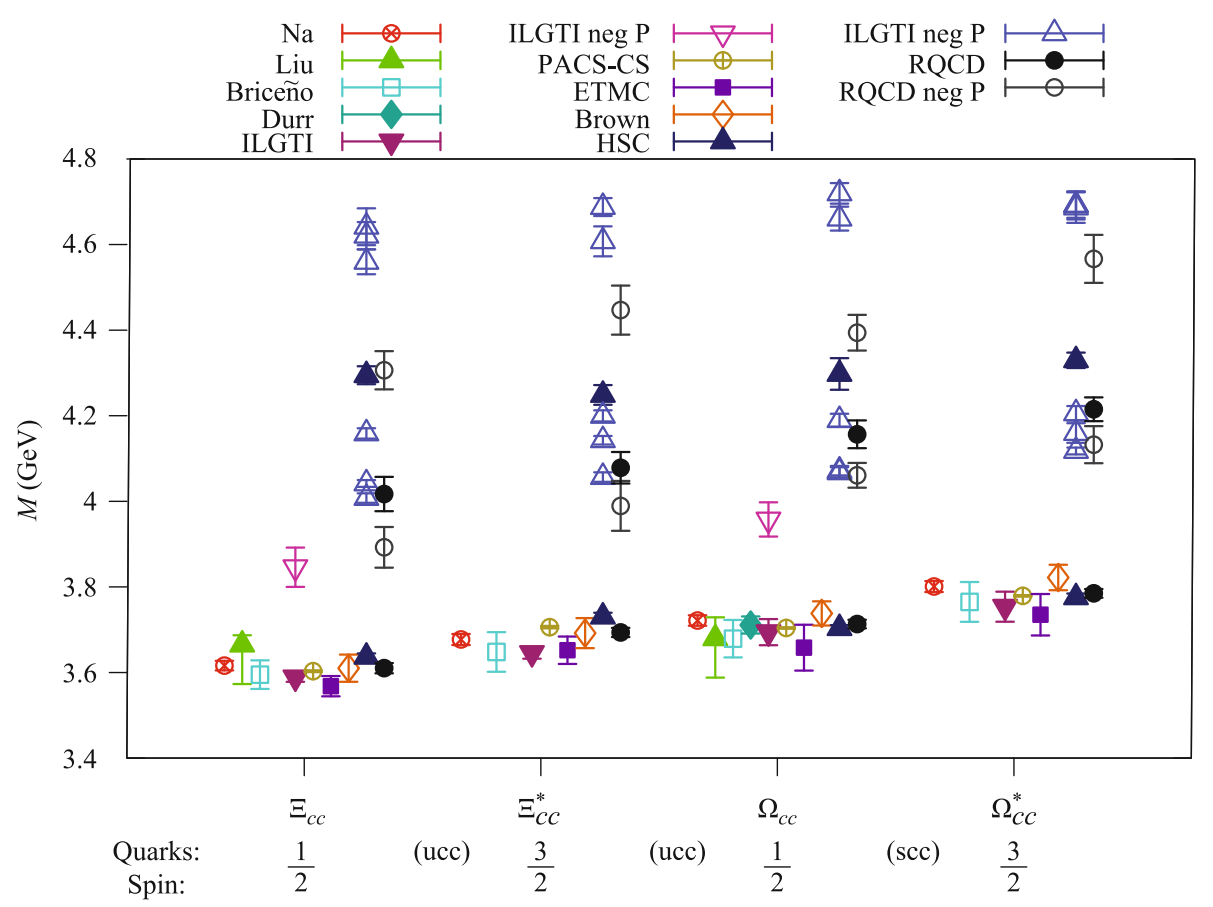

Fig. 4 Doubly charmed low-lying baryon spectra taken from Ref. [57].

quark and chiral symmetry of the light quarks will have interesting implications for the low-energy dynamics of heavy baryons interacting with Goldstone bosons.

The strong decays of charmed baryons are most conveniently described by the HHChPT, into which heavy quark symmetry and chiral symmetry are incorporated [68-70]. Heavy baryon chiral Lagrangians were first constructed in Ref. [68] for strong decays of $s$-wave charmed baryons and in Refs. $[9,14]$ for $p$-wave ones. Previous phenomenological studies of the strong decays of $p$-wave charmed baryons based on HHChPT can be found in Refs. [9, 10, 14, 71, 72]. The chiral Lagrangian involves two coupling constants, $g_{1}$ and $g_{2}$, for $P$-wave transitions between $s$-wave and $s$-wave baryons [68]; six couplings, $h_{2}-h_{7}$, for the $S$-wave transitions between $s$-wave and $p$-wave baryons; and eight couplings; $h_{8}-h_{15}$, for the $D$-wave transitions between $s$-wave and $p$-wave baryons [9]. The general chiral Lagrangian for heavy baryons coupled to pseudoscalar mesons can be expressed compactly in terms of superfields. We will not write the relevant Lagrangians here; instead the reader is referred to Eqs. (3.1) and (3.3) of Ref. [9]. The partial widths relevant for our purposes are [9]

$$
\begin{aligned}
\Gamma\left(\Sigma_{c}^{*} \rightarrow \Sigma_{c} \pi\right) & =\frac{g_{1}^{2}}{2 \pi f_{\pi}^{2}} \frac{m_{\Sigma_{c}}}{m_{\Sigma_{c}^{*}}} p_{\pi}^{3}, \\
\Gamma\left(\Sigma_{c} \rightarrow \Lambda_{c} \pi\right) & =\frac{g_{2}^{2}}{2 \pi f_{\pi}^{2}} \frac{m_{\Lambda_{c}}}{m_{\Sigma_{c}}} p_{\pi}^{3},
\end{aligned}
$$

$$
\begin{aligned}
& \Gamma\left(\Lambda_{c 1}\left(1 / 2^{-}\right) \rightarrow \Sigma_{c} \pi\right)=\frac{h_{2}^{2}}{2 \pi f_{\pi}^{2}} \frac{m_{\Sigma_{c}}}{m_{\Lambda_{c 1}}} E_{\pi}^{2} p_{\pi}, \\
& \Gamma\left(\Sigma_{c 0}\left(1 / 2^{-}\right) \rightarrow \Lambda_{c} \pi\right)=\frac{h_{3}^{2}}{2 \pi f_{\pi}^{2}} \frac{m_{\Lambda_{c}}}{m_{\Sigma_{c 0}}} E_{\pi}^{2} p_{\pi}, \\
& \Gamma\left(\Lambda_{c 1}\left(3 / 2^{-}\right) \rightarrow \Sigma_{c} \pi\right)=\frac{2 h_{8}^{2}}{9 \pi f_{\pi}^{2}} \frac{m_{\Sigma_{c}}}{m_{\Lambda_{c 1}(3 / 2)}} p_{\pi}^{5}, \\
& \Gamma\left(\Sigma_{c 1}\left(3 / 2^{-}\right) \rightarrow \Sigma_{c}^{(*)} \pi\right)=\frac{h_{9}^{2}}{9 \pi f_{\pi}^{2}} \frac{m_{\Sigma_{c}^{*}}}{m_{\Sigma_{c 1}(3 / 2)}} p_{\pi}^{5}, \\
& \Gamma\left(\Sigma_{c 2}\left(3 / 2^{-}\right) \rightarrow \Lambda_{c} \pi\right)=\frac{4 h_{10}^{2}}{15 \pi f_{\pi}^{2}} \frac{m_{\Lambda_{c}}}{m_{\Sigma_{c 2}}} p_{\pi}^{5}, \\
& \Gamma\left(\Sigma_{c 2}\left(3 / 2^{-}\right) \rightarrow \Sigma_{c}^{(*)} \pi\right)=\frac{h_{11}^{2}}{10 \pi f_{\pi}^{2}} \frac{m_{\Sigma_{c}^{(*)}}}{m_{\Sigma_{c 2}}} p_{\pi}^{5}, \\
& \Gamma\left(\Sigma_{c 2}\left(5 / 2^{-}\right) \rightarrow \Sigma_{c} \pi\right)=\frac{2 h_{11}^{2}}{45 \pi f_{\pi}^{2}} \frac{m_{\Sigma_{c}}}{m_{\Sigma_{c 2}}} p_{\pi}^{5}, \\
& \Gamma\left(\Sigma_{c 2}\left(5 / 2^{-}\right) \rightarrow \Sigma_{c}^{*} \pi\right)=\frac{7 h_{11}^{2}}{45 \pi f_{\pi}^{2}} \frac{m_{\Sigma_{c}^{*}}}{m_{\Sigma_{c 2}}} p_{\pi}^{5},
\end{aligned}
$$

where $f_{\pi}=132 \mathrm{MeV}$. The dependence on the pion momentum is proportional to $p_{\pi}, p_{\pi}^{3}$ and $p_{\pi}^{5}$ for $S$-wave, $P$-wave and $D$-wave transitions, respectively. It is obvious that the couplings $g_{1}, g_{2}, h_{2}, \cdots, h_{7}$ are dimensionless, whereas $h_{8}, \cdots, h_{15}$ have canonical dimension $E^{-1}$.

\subsection{Strong decays of $s$-wave charmed baryons}

Because the strong decay $\Sigma_{c}^{*} \rightarrow \Sigma_{c} \pi$ is kinematically prohibited, the coupling $g_{1}$ cannot be extracted directly 
Table 6 Decay widths (in units of $\mathrm{MeV}$ ) of $s$-wave charmed baryons where the measured rates are taken from 2006 PDG [73].

\begin{tabular}{ccc}
\hline Decay & Expt. & HHChPT \\
\hline$\Sigma_{c}^{++} \rightarrow \Lambda_{c}^{+} \pi^{+}$ & $2.23 \pm 0.30$ & input \\
$\Sigma_{c}^{+} \rightarrow \Lambda_{c}^{+} \pi^{0}$ & $<4.6$ & $2.6 \pm 0.4$ \\
$\Sigma_{c}^{0} \rightarrow \Lambda_{c}^{+} \pi^{-}$ & $2.2 \pm 0.4$ & $2.2 \pm 0.3$ \\
$\Sigma_{c}(2520)^{++} \rightarrow \Lambda_{c}^{+} \pi^{+}$ & $14.9 \pm 1.9$ & $16.7 \pm 2.3$ \\
$\Sigma_{c}(2520)^{+} \rightarrow \Lambda_{c}^{+} \pi^{0}$ & $<17$ & $17.4 \pm 2.3$ \\
$\Sigma_{c}(2520)^{0} \rightarrow \Lambda_{c}^{+} \pi^{-}$ & $16.1 \pm 2.1$ & $16.6 \pm 2.2$ \\
$\Xi_{c}(2645)^{+} \rightarrow \Xi_{c}^{0,+} \pi^{+, 0}$ & $<3.1$ & $2.8 \pm 0.4$ \\
$\Xi_{c}(2645)^{0} \rightarrow \Xi_{c}^{+, 0} \pi^{-, 0}$ & $<5.5$ & $2.9 \pm 0.4$ \\
\hline
\end{tabular}

from the strong decays of heavy baryons. In the framework of HHChPT, one can use some measurements as input to fix the coupling $g_{2}$, which, in turn, can be used to predict the rates of other strong decays. Among the strong decays $\Sigma_{c}^{(*)} \rightarrow \Lambda_{c} \pi, \Sigma_{c}^{++} \rightarrow \Lambda_{c}^{+} \pi^{+}$is the most well-measured. Hence, we shall use this mode to extract the coupling $g_{2}$. Using the 2006 data [73] $\Gamma\left(\Sigma_{c}^{++}\right)=$ $\Gamma\left(\Sigma_{c}^{++} \rightarrow \Lambda_{c}^{+} \pi^{+}\right)=2.23 \pm 0.30 \mathrm{MeV}$, the coupling $g_{2}$ is extracted as

$$
\left|g_{2}\right|_{2006}=0.605_{-0.043}^{+0.039} \text {. }
$$

The predicted rates of other modes are shown in Table 6 . The agreement between theory and experiment is clearly excellent, except that the predicted width for $\Sigma_{c}^{*++} \rightarrow \Lambda_{c}^{+} \pi^{+}$is slightly too large.

Using the new data from the 2014 Particle Data Group [8] in conjunction with the new measurements of the $\Sigma_{c}$ and $\Sigma_{c}^{*}$ widths by Belle [11], we obtain the new average $\Gamma\left(\Sigma_{c}^{++} \rightarrow \Lambda_{c}^{+} \pi^{+}\right)=1.94_{-0.16}^{+0.08} \mathrm{MeV}$ (see Table 3 ). Therefore, the coupling $g_{2}$ is reduced to

$$
\left|g_{2}\right|_{2015}=0.565_{-0.024}^{+0.011} \text {. }
$$

From Table 7 we see that the agreement between theory and experiment is further improved: The predicted $\Xi_{c}(2645)^{+}$width is consistent with the first new measurement by Belle [12], and the new calculated width for $\Sigma_{c}^{*++} \rightarrow \Lambda_{c}^{+} \pi^{+}$is now in agreement with experiment. It is also clear that the $\Sigma_{c}$ width is smaller than that of $\Sigma_{c}^{*}$ by a factor of $\sim 7$, although they will become the same in the limit of heavy quark symmetry.

\subsection{Strong decays of $p$-wave charmed baryons}

Because $\Lambda_{c}(2595)^{+}$and $\Lambda_{c}(2625)^{+}$form the doublet $\Lambda_{c 1}\left(\frac{1}{2}^{-}, \frac{3}{2}^{-}\right)$, it appears from Eq. (3.1) that the couplings $h_{2}$ and $h_{8}$ can in principle be extracted from $\Lambda_{c}(2595) \rightarrow \Sigma_{c} \pi$ and from $\Lambda_{c}(2625) \rightarrow \Sigma_{c} \pi$, respectively. Likewise, the information on the couplings $h_{10}$ and $h_{11}$ can be inferred from the strong decays of $\Sigma_{c}(2800)$ identified with $\Sigma_{c 2}\left(3 / 2^{-}\right)$. Couplings other than $h_{2}, h_{8}$, and $h_{10}$ can be related to each other via the quark model [9].

Although the coupling $h_{2}$ can be inferred from the twobody decay $\Lambda_{c}(2595) \rightarrow \Sigma_{c} \pi$, this method is less accurate because this decay is kinematically barely allowed or even prohibited, depending on the mass of $\Lambda_{c}(2595)^{+}$. For the old mass measurement $m\left(\Lambda_{c}(2595)\right)=2595.4 \pm 0.6 \mathrm{MeV}$

Table 8 Decay widths (in units of $\mathrm{MeV}$ ) of $p$-wave charmed baryons where the measured rates are taken from 2006 PDG [73].

\begin{tabular}{ccc}
\hline Decay & $\begin{array}{c}\text { Expt. } \\
{[73]}\end{array}$ & $\begin{array}{c}\text { HHChPT } \\
{[10]}\end{array}$ \\
\hline$\Lambda_{c}(2595)^{+} \rightarrow\left(\Lambda_{c}^{+} \pi \pi\right)_{R}$ & $2.63_{-1.09}^{+1.56}$ & input \\
$\Lambda_{c}(2595)^{+} \rightarrow \Sigma_{c}^{++} \pi^{-}$ & $0.65_{-0.31}^{+0.41}$ & $0.72_{-0.30}^{+0.43}$ \\
$\Lambda_{c}(2593)^{+} \rightarrow \Sigma_{c}^{0} \pi^{+}$ & $0.67_{-0.31}^{+0.41}$ & $0.77_{-0.32}^{+0.36}$ \\
$\Lambda_{c}(2593)^{+} \rightarrow \Sigma_{c}^{+} \pi^{0}$ & & $1.57_{-0.65}^{+0.93}$ \\
$\Lambda_{c}(2625)^{+} \rightarrow \Sigma_{c}^{++} \pi^{-}$ & $<0.10$ & 0.029 \\
$\Lambda_{c}(2625)^{+} \rightarrow \Sigma_{c}^{0} \pi^{+}$ & $<0.09$ & 0.029 \\
$\Lambda_{c}(2625)^{+} \rightarrow \Sigma_{c}^{+} \pi^{0}$ & & 0.041 \\
$\Lambda_{c}(2625)^{+} \rightarrow \Lambda_{c}^{+} \pi \pi$ & $<1.9$ & 0.21 \\
$\Sigma_{c}(2800)^{++} \rightarrow \Lambda_{c} \pi, \Sigma_{c}^{(*)} \pi$ & $75_{-17}^{+22}$ & input \\
$\Sigma_{c}(2800)^{+} \rightarrow \Lambda_{c} \pi, \Sigma_{c}^{(*)} \pi$ & $62_{-40}^{+60}$ & input \\
$\Sigma_{c}(2800)^{0} \rightarrow \Lambda_{c} \pi, \Sigma_{c}^{(*)} \pi$ & $61_{-18}^{+28}$ & input \\
$\Xi_{c}(2790)^{+} \rightarrow \Xi_{c}^{\prime 0,+} \pi^{+, 0}$ & $<15$ & $8.0_{-3.3}^{+4.3}$ \\
$\Xi_{c}(2790)^{0} \rightarrow \Xi_{c}^{\prime+, 0} \pi^{-, 0}$ & $<12$ & $8.5_{-3.5}^{+5.0}$ \\
$\Xi_{c}(2815)^{+} \rightarrow \Xi_{c}^{*+, 0} \pi^{0,+}$ & $<3.5$ & $3.4_{-1.4}^{+2.0}$ \\
$\Xi_{c}(2815)^{0} \rightarrow \Xi_{c}^{*+, 0} \pi^{-, 0}$ & $<6.5$ & $3.6_{-1.5}^{+2.1}$ \\
\hline
\end{tabular}

Table 7 Decay widths (in units of $\mathrm{MeV}$ ) of $s$-wave charmed baryons. Data are taken from 2014 PDG [8] together with the new

\begin{tabular}{|c|c|c|c|c|c|c|}
\hline Decay & $\begin{array}{c}\text { Expt. } \\
{[8]}\end{array}$ & $\mathrm{HHChPT}$ & $\begin{array}{c}\text { Tawfiq } \\
\text { et al. }[74]\end{array}$ & $\begin{array}{c}\text { Ivanov } \\
\text { et al. }[75]\end{array}$ & $\begin{array}{c}\text { Huang } \\
\text { et al. }[76]\end{array}$ & $\begin{array}{l}\text { Albertus } \\
\text { et al. [78] }\end{array}$ \\
\hline$\Sigma_{c}^{++} \rightarrow \Lambda_{c}^{+} \pi^{+}$ & $1.94_{-0.16}^{+0.08}$ & input & $1.51 \pm 0.17$ & $2.85 \pm 0.19$ & 2.5 & $2.41 \pm 0.07$ \\
\hline$\Sigma_{c}^{+} \rightarrow \Lambda_{c}^{+} \pi^{0}$ & $<4.6$ & $2.3_{-0.2}^{+0.1}$ & $1.56 \pm 0.17$ & $3.63 \pm 0.27$ & 3.2 & $2.79 \pm 0.08$ \\
\hline$\Sigma_{c}^{0} \rightarrow \Lambda_{c}^{+} \pi^{-}$ & $1.9_{-0.2}^{+0.1}$ & $1.9_{-0.2}^{+0.1}$ & $1.44 \pm 0.16$ & $2.65 \pm 0.19$ & 2.4 & $2.37 \pm 0.07$ \\
\hline$\Sigma_{c}(2520)^{++} \rightarrow \Lambda_{c}^{+} \pi^{+}$ & $14.8_{-0.4}^{+0.3}$ & $14.5_{-0.8}^{+0.5}$ & $11.77 \pm 1.27$ & $21.99 \pm 0.87$ & 8.2 & $17.52 \pm 0.75$ \\
\hline$\Sigma_{c}(2520)^{+} \rightarrow \Lambda_{c}^{+} \pi^{0}$ & $<17$ & $15.2_{-1.3}^{+0.6}$ & & & 8.6 & $17.31 \pm 0.74$ \\
\hline$\Xi_{c}(2645)^{0} \rightarrow \Xi_{c}^{+, 0} \pi^{-, 0}$ & $<5.5$ & $2.5_{-0.2}^{+0.1}$ & $1.83 \pm 0.06$ & $3.12 \pm 0.33$ & & $3.03 \pm 0.10$ \\
\hline
\end{tabular}
measurements of $\Sigma_{c}, \Sigma_{c}^{*}$ [11] and $\Xi_{c}(2645)^{+}$widths [12]. Theoretical predictions of [74] are taken from Table IV of [75]. 
Table 9 Decay widths (in units of $\mathrm{MeV}$ ) of $p$-wave charmed baryons. Data are taken from 2014 PDG [8] together with the new measurements of $\Sigma_{c}, \Sigma_{c}^{*}$ [11] and $\Xi_{c}(2645)^{+}$widths [12]. Theoretical predictions of [74] are taken from Table IV of [75].

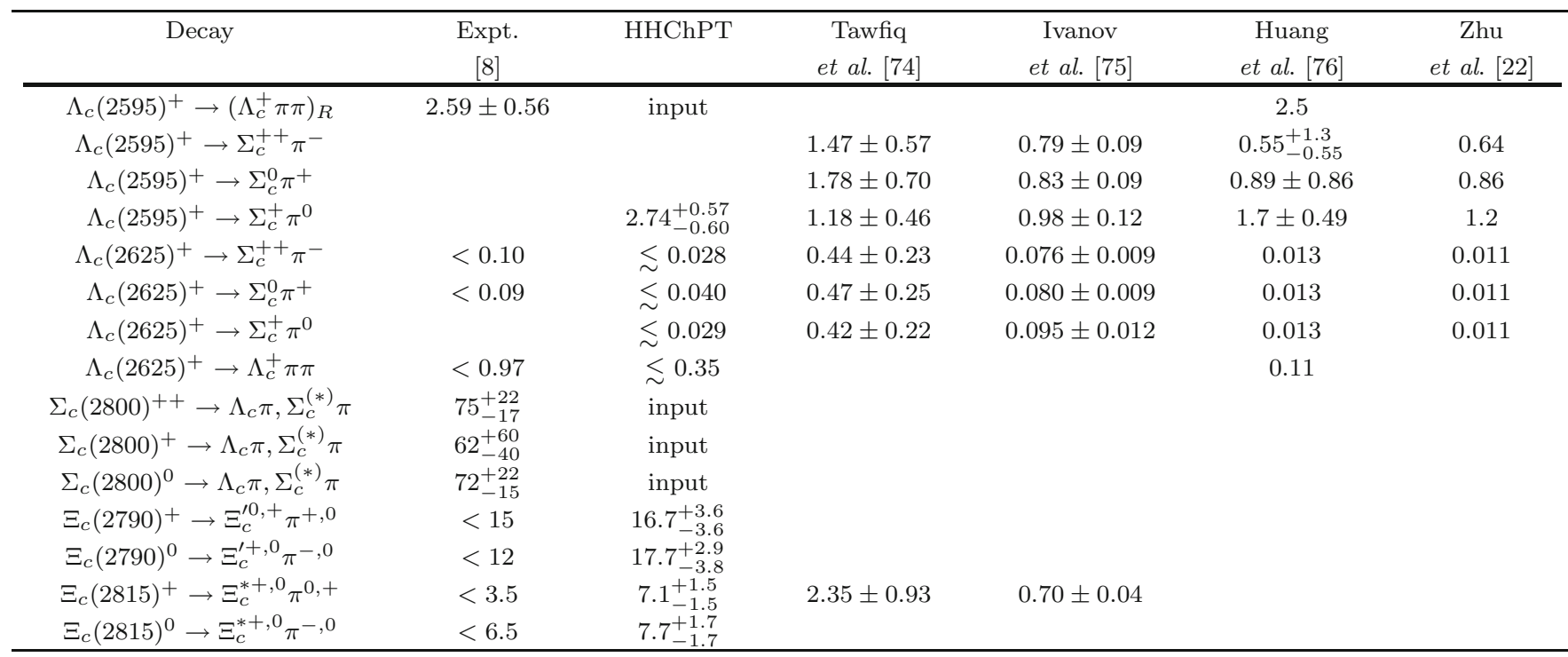

[73], $\Lambda_{c}(2595)^{+} \rightarrow \Sigma_{c}^{++} \pi^{-}, \Sigma_{c}^{0} \pi^{+}$and $\Lambda_{c}(2595)^{+} \rightarrow$ $\Sigma^{+} \pi^{0}$ are kinematically barely allowed. However, for the new measurement by the CDF, $m\left(\Lambda_{c}(2595)\right)=2592.25 \pm$ $0.28 \mathrm{MeV}$ [79], only the last mode is allowed. Moreover, the finite width effect of the intermediate resonant states could become important [71].

We next turn to the three-body decays $\Lambda_{c}^{+} \pi \pi$ of $\Lambda_{c}(2595)^{+}$and $\Lambda_{c}(2625)^{+}$to extract $h_{2}$ and $h_{8}$. As shown in Ref. [10], the 2006 data $\Gamma\left(\Lambda_{c}(2595)\right)=3.6_{-1.3}^{+2.0} \mathrm{MeV}$ [73] and for the $\Lambda_{c}(2595)$ mass lead to the resonant rate [10]

$$
\Gamma\left(\Lambda_{c}(2593)^{+} \rightarrow \Lambda_{c}^{+} \pi \pi\right)_{R}=\left(2.63_{-1.09}^{+1.56}\right) \mathrm{MeV},
$$

as shown in Table 9. Assuming the pole contributions to $\Lambda_{c}(2595)^{+} \rightarrow \Lambda_{c}^{+} \pi \pi$ due to the intermediate states $\Sigma_{c}$ and $\Sigma_{c}^{*}$, the resonant rate for the process $\Lambda_{c_{1}}^{+}(2595) \rightarrow$ $\Lambda_{c}^{+} \pi^{+} \pi^{-}$can be calculated in the framework of HHChPT [9]. Numerically, we found

$$
\begin{aligned}
& \Gamma\left(\Lambda_{c}(2595)^{+} \rightarrow \Lambda_{c}^{+} \pi \pi\right)_{R} \\
& \quad=13.82 h_{2}^{2}+26.28 h_{8}^{2}-2.97 h_{2} h_{8}, \\
& \Gamma\left(\Lambda_{c}(2625)^{+} \rightarrow \Lambda_{c}^{+} \pi \pi\right)_{R} \\
& \quad=0.617 h_{2}^{2}+0.136 \times 10^{6} h_{8}^{2}-27 h_{2} h_{8},
\end{aligned}
$$

where $\Lambda_{c}^{+} \pi \pi=\Lambda_{c}^{+} \pi^{+} \pi^{-}+\Lambda_{c}^{+} \pi^{0} \pi^{0}$. It is clear that the limit on $\Gamma\left(\Lambda_{c}(2625)\right)$ gives an upper bound on $h_{8}$ of the order of $10^{-3}$ (in units of $\mathrm{MeV}^{-1}$ ), whereas the decay width of $\Lambda_{c}(2595)$ is entirely governed by the coupling $h_{2}$. Specifically, we have [10]

$$
\begin{aligned}
\left|h_{2}\right|_{2006} & =0.437_{-0.102}^{+0.114}, \\
\left|h_{8}\right|_{2006} & <3.65 \times 10^{-3} \mathrm{MeV}^{-1} .
\end{aligned}
$$

It has been noted [72] that the proximity of the $\Lambda_{c}(2595)^{+}$mass to the sum of the masses of its decay products will lead to an important threshold effect that will lower the $\Lambda_{c}(2595)^{+}$mass by $2-3 \mathrm{MeV}$ compared to the observed mass. A more sophisticated treatment of the mass lineshape of $\Lambda_{c}(2595)^{+} \rightarrow \Lambda_{c}^{+} \pi^{+} \pi^{-}$by the CDF yields $m\left(\Lambda_{c}(2595)\right)=2592.25 \pm 0.28 \mathrm{MeV}$ [79], which is $3.1 \mathrm{MeV}$ smaller than the 2006 world average. Therefore, the strong decay $\Lambda_{c}(2595) \rightarrow \Lambda_{c} \pi \pi$ is very close to the threshold. With the new measurement of $m\left(\Lambda_{c}(2595)\right.$ ), we have (in units of $\mathrm{MeV}$ ) [32]

$$
\begin{aligned}
& \Gamma\left(\Lambda_{c}(2595)^{+} \rightarrow \Lambda_{c}^{+} \pi \pi\right)_{R} \\
& \quad=g_{2}^{2}\left(20.45 h_{2}^{2}+43.92 h_{8}^{2}-8.95 h_{2} h_{8}\right) \\
& \Gamma\left(\Lambda_{c}(2625)^{+} \rightarrow \Lambda_{c}^{+} \pi \pi\right)_{R} \\
& \quad=g_{2}^{2}\left(1.78 h_{2}^{2}+4.557 \times 10^{6} h_{8}^{2}-79.75 h_{2} h_{8}\right) .
\end{aligned}
$$

By fitting to the measured $M\left(p K^{-} \pi^{+} \pi^{+}\right)-$ $M\left(p K^{-} \pi^{+}\right)$mass difference distributions and using $g_{2}^{2}=$ 0.365 , CDF obtained $h_{2}^{2}=0.36 \pm 0.08$ or $\left|h_{2}\right|=0.60 \pm 0.07$ [79]. This corresponds to a decay width $\Gamma\left(\Lambda_{c}(2595)^{+}\right)=$ $2.59 \pm 0.30 \pm 0.47 \mathrm{MeV}$ [79]. For the width of $\Lambda_{c}(2625)^{+}$, CDF observed a value consistent with zero and therefore calculated an upper limit of $0.97 \mathrm{MeV}$ using a Bayesian approach. From the CDF measurements $\Gamma\left(\Lambda_{c}(2595)^{+}\right)=$ $2.59 \pm 0.56 \mathrm{MeV}$ and $\Gamma\left(\Lambda_{c}(2625)^{+}\right)<0.97 \mathrm{MeV}$, we obtain

$$
\begin{aligned}
& \left|h_{2}\right|_{2015}=0.63 \pm 0.07, \\
& \left|h_{8}\right|_{2015}<2.32 \times 10^{-3} \mathrm{MeV}^{-1} .
\end{aligned}
$$

Hence, the magnitude of the coupling $h_{2}$ is greatly en- 
hanced from 0.437 to 0.63 . Our $h_{2}$ differs slightly from the value of 0.60 obtained by CDF. This is because the CDF used $\left|g_{2}\right|=0.604$ to calculate the mass dependence of $\Gamma\left(\Lambda_{c}^{+} \pi \pi\right)$, whereas we used $\left|g_{2}\right|=0.565$.

The large difference between the values of the coupling $h_{2}$ obtained in 2006 and 2015 is ascribed to the fact that the mass of $\Lambda_{c}(2595)^{+}$is $3.1 \mathrm{MeV}$ lower than the previous world average because of the threshold effect. To illustrate this, we consider the dependence of $\Gamma\left(\Lambda_{c}^{+} \pi^{+} \pi^{-}\right) / h_{2}^{2}$ and $\Gamma\left(\Lambda_{c}^{+} \pi^{0} \pi^{0}\right) / h_{2}^{2}$ on $\Delta M\left(\Lambda_{c}(2595)\right) \equiv M\left(\Lambda_{c}(2595)^{+}\right)-M\left(\Lambda_{c}^{+}\right)$as depicted in Fig. 5. $\Gamma\left(\Lambda_{c}^{+} \pi \pi\right) / h_{2}^{2}$ at $\Delta M\left(\Lambda_{c}(2595)\right)=305.79 \mathrm{MeV}$ is clearly smaller than that at $308.9 \mathrm{MeV}$. This explains why $h_{2}$ should become larger when $\Delta M\left(\Lambda_{c}(2595)\right)$ becomes smaller.

The $\Xi_{c}(2790)$ and $\Xi_{c}(2815)$ baryons form the doublet $\Xi_{c 1}\left(\frac{1}{2}^{-}, \frac{3}{2}^{-}\right)$. The $\Xi_{c}(2790)$ and $\Xi_{c}(2815)$ widths predicted using the coupling $h_{2}$ obtained from Eq. (3.8) and assuming SU(3) flavor symmetry are shown in Table 9 . The predicted two-body decay rates of $\Xi_{c}(2790)^{0}$ and $\Xi_{c}(2815)^{+}$clearly exceed the current experimental limits because of the enhancement of $h_{2}$ (see Table 9). Hence, there is a tension for the coupling $h_{2}$, as its value extracted from from $\Lambda_{c}(2595)^{+} \rightarrow \Lambda_{c}^{+} \pi \pi$ will imply $\Xi_{c}(2790)^{0} \rightarrow \Xi_{c}^{\prime} \pi$ and $\Xi_{c}(2815)^{+} \rightarrow \Xi_{c}^{*} \pi$ rates slightly above current limits. It is conceivable that $\mathrm{SU}(3)$ flavor symmetry breaking can help account for the discrepancy.

Some information on the coupling $h_{10}$ can be inferred from the strong decays of $\Sigma_{c}(2800)$. From Eq. (3.1) and the quark model relation $\left|h_{3}\right|=\sqrt{3}\left|h_{2}\right|$ from Ref. [9], we obtain, for example, $\Gamma\left(\Sigma_{c 0}^{++} \rightarrow \Lambda_{c}^{+} \pi^{+}\right) \approx 885 \mathrm{MeV}$. Hence, $\Sigma_{c}(2800)$ cannot be identified with $\Sigma_{c 0}\left(1 / 2^{-}\right)$. Using the quark model relation $h_{11}^{2}=2 h_{10}^{2}$ and the measured widths of $\Sigma_{c}(2800)^{++,+, 0}$ (Table 3), we obtain

$$
\left|h_{10}\right|=\left(0.85_{-0.08}^{+0.11}\right) \times 10^{-3} \mathrm{MeV}^{-1} \text {. }
$$

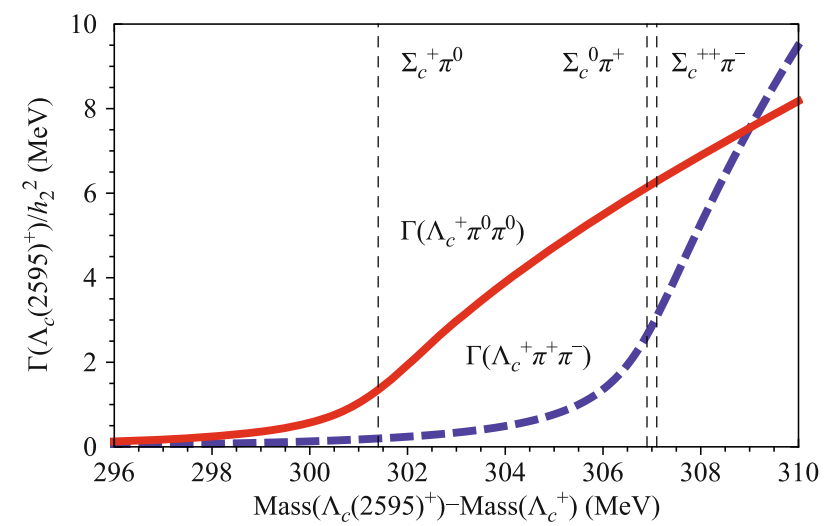

Fig. 5 Calculated dependence of $\Gamma\left(\Lambda_{c}^{+} \pi^{0} \pi^{0}\right) / h_{2}^{2}$ (full curve) and $\Gamma\left(\Lambda_{c}^{+} \pi^{+} \pi^{-}\right) / h_{2}^{2}$ (dashed curve) on $m\left(\Lambda_{c}(2595)^{+}\right)-m\left(\Lambda_{c}^{+}\right)$, where we have used the parameters $g_{2}=0.565, h_{2}=0.63$, and $h_{8}=0.85 \times 10^{-3} \mathrm{MeV}^{-1}$.
The quark model relation $\left|h_{8}\right|=\left|h_{10}\right|$ then leads to

$$
\left|h_{8}\right| \approx\left(0.85_{-0.08}^{+0.11}\right) \times 10^{-3} \mathrm{MeV}^{-1},
$$

which improves the previous limit (3.8) by a factor of 3 . The calculated partial widths of $\Lambda_{c}(2625)^{+}$shown in Table 9 are consistent with experimental limits.

\section{Lifetimes}

\subsection{Singly charmed baryons}

Among singly charmed baryons, the antitriplet states $\Lambda_{c}^{+}, \Xi_{c}^{+}, \Xi_{c}^{0}$, and the $\Omega_{c}^{0}$ baryon in the sextet decay weakly. In 2006, the world averages of their lifetimes were [73]

$$
\begin{aligned}
& \tau\left(\Lambda_{c}^{+}\right)=(200 \pm 6) \times 10^{-15} \mathrm{~s}, \\
& \tau\left(\Xi_{c}^{+}\right)=(442 \pm 26) \times 10^{-15} \mathrm{~s}, \\
& \tau\left(\Xi_{c}^{0}\right)=\left(112_{-10}^{+13}\right) \times 10^{-15} \mathrm{~s}, \\
& \tau\left(\Omega_{c}^{0}\right)=(69 \pm 12) \times 10^{-15} \mathrm{~s} .
\end{aligned}
$$

These results remain the same even in 2014 [8]. As we shall see below, the lifetime hierarchy $\tau\left(\Xi_{c}^{+}\right)>\tau\left(\Lambda_{c}^{+}\right)>$ $\tau\left(\Xi_{c}^{0}\right)>\tau\left(\Omega_{c}^{0}\right)$ is understood qualitatively but not quantitatively in the operator product expansion (OPE) approach.

On the basis of the OPE approach to analysis of inclusive weak decays, the inclusive rate of the charmed baryon is schematically represented by

$$
\begin{aligned}
\Gamma\left(\mathcal{B}_{c} \rightarrow f\right) & =\frac{G_{F}^{2} m_{c}^{5}}{192 \pi^{3}} V_{\mathrm{CKM}} \\
& \cdot\left(A_{0}+\frac{A_{2}}{m_{c}^{2}}+\frac{A_{3}}{m_{c}^{3}}+\mathcal{O}\left(\frac{1}{m_{c}^{4}}\right)\right),
\end{aligned}
$$

where $V_{\text {CKM }}$ is the relevant Cabibbo-KobayashiMaskawa matrix element. The $A_{0}$ term comes from the $c$ quark decay and is common to all charmed hadrons. There are no linear $1 / m_{Q}$ corrections to the inclusive decay rate owing to the lack of gauge-invariant dimensionfour operators [81-84], a consequence known as Luke's theorem [85]. Nonperturbative corrections start at order $1 / m_{Q}^{2}$ and are model-independent. Spectator effects in inclusive decays due to the Pauli interference and $W$ exchange contributions account for the $1 / m_{c}^{3}$ corrections and they have two noteworthy features: First, the estimate of the spectator effects is model dependent; the hadronic four-quark matrix elements are usually evaluated by assuming the factorization approximation for mesons and the quark model for baryons. Second, there is a two-body phase-space enhancement factor of $16 \pi^{2}$ for spectator effects relative to the three-body phase space 
Table 10 Various contributions to the decay rates (in units of $10^{-12} \mathrm{GeV}$ ) of singly charmed baryons [86]. Experimental values are taken from Ref. [8].

\begin{tabular}{ccccccccc}
\hline & $\Gamma^{\text {dec }}$ & $\Gamma^{\text {ann }}$ & $\Gamma_{-}^{\text {int }}$ & $\Gamma_{+}^{\text {int }}$ & $\Gamma_{\mathrm{SL}}$ & $\Gamma^{\text {tot }}$ & $\tau\left(10^{-13} \mathrm{~s}\right)$ & $\tau_{\text {expt }}\left(10^{-13} \mathrm{~s}\right)$ \\
\hline$\Lambda_{c}^{+}$ & 1.006 & 1.342 & -0.196 & & 0.323 & 2.492 & 2.64 & $2.00 \pm 0.06$ \\
$\Xi_{c}^{+}$ & 1.006 & 0.071 & -0.203 & 0.364 & 0.547 & 1.785 & 3.68 & $4.42 \pm 0.26$ \\
$\Xi_{c}^{0}$ & 1.006 & 1.466 & & 0.385 & 0.547 & 3.404 & 1.93 & $1.12_{-0.10}^{+0.13}$ \\
$\Omega_{c}^{0}$ & 1.132 & 0.439 & & 1.241 & 1.039 & 3.851 & 1.71 & $0.69 \pm 0.12$ \\
\hline
\end{tabular}

for heavy quark decay. This implies that spectator effects, which are of the order of $1 / m_{c}^{3}$, are comparable to and even exceed the $1 / m_{c}^{2}$ terms.

The total width of the charmed baryon $\mathcal{B}_{c}$ generally receives contributions from inclusive nonleptonic and semileptonic decays: $\Gamma\left(\mathcal{B}_{c}\right)=\Gamma_{\mathrm{NL}}\left(\mathcal{B}_{c}\right)+\Gamma_{\mathrm{SL}}\left(\mathcal{B}_{c}\right)$. The nonleptonic contribution can be decomposed into

$$
\Gamma_{\mathrm{NL}}\left(\mathcal{B}_{c}\right)=\Gamma^{\mathrm{dec}}\left(\mathcal{B}_{c}\right)+\Gamma^{\mathrm{ann}}\left(\mathcal{B}_{c}\right)+\Gamma_{-}^{\mathrm{int}}\left(\mathcal{B}_{c}\right)+\Gamma_{+}^{\mathrm{int}}\left(\mathcal{B}_{c}\right),
$$

corresponding to the $c$-quark decay, $W$-exchange contribution, and destructive and constructive Pauli interference. The inclusive decay rate is known to be governed by the imaginary part of an effective nonlocal forward transition operator $T$. Therefore, $\Gamma^{\operatorname{dec}}$ corresponds to the imaginary part of Fig. 6(a) sandwiched between the same $\mathcal{B}_{c}$ states. At the Cabibbo-allowed level, $\Gamma^{\text {dec }}$ represents the decay rate of $c \rightarrow s u \bar{d}$, and $\Gamma^{\text {ann }}$ denotes the contribution from the $W$-exchange diagram $c d \rightarrow u s$. The interference $\Gamma_{-}^{\text {int }}\left(\Gamma_{+}^{\text {int }}\right)$ arises from destructive (constructive) interference between the $u(s)$ quark produced in $c$ quark decay and the spectator $u(s)$ quark in the charmed baryon $\mathcal{B}_{c}$. Note that the constructive Pauli interference is unique to the charmed baryon sector, as it does not occur in the bottom sector. From the quark content of the charmed baryons, it is clear that at the Cabibboallowed level, destructive interference occurs in $\Lambda_{c}^{+}$and $\Xi_{c}^{+}$decays (Fig. $6(\mathrm{c})$ ), whereas $\Xi_{c}^{+}, \Xi_{c}^{0}$ and $\Omega_{c}^{0}$ can have constructive interference $\Gamma_{+}^{\text {int }}$ (Fig. $\left.6(\mathrm{~d})\right)$. Because $\Omega_{c}^{0}$ (a)

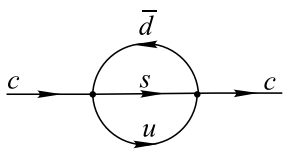

(c)

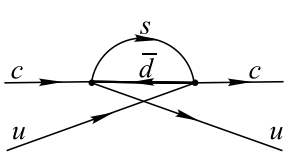

(b)

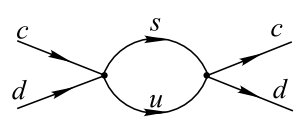

(d)

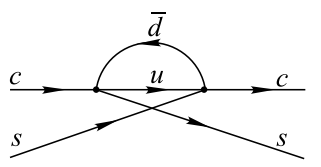

Fig. 6 Contributions to nonleptonic decay rates of charmed baryons from four-quark operators: (a) c-quark decay, (b) Wexchange, (c) destructive Pauli interference, and (d) constructive interference. contains two $s$ quarks, it is natural to expect that $\Gamma_{+}^{\text {int }}\left(\Omega_{c}^{0}\right) \gg \Gamma_{+}^{\text {int }}\left(\Xi_{c}\right)$. The $W$-exchange contribution (Fig. $6(\mathrm{~b}))$ occurs only for $\Xi_{c}^{0}$ and $\Lambda_{c}^{+}$at the same Cabibboallowed level. In the heavy quark expansion approach, the above-mentioned spectator effects can be described in terms of the matrix elements of local four-quark operators.

The inclusive nonleptonic rates of charmed baryons in the valence quark approximation and in the limit $m_{s} / m_{c}=0$ are expressed as [86]:

$$
\begin{aligned}
& \Gamma_{\mathrm{NL}}\left(\Lambda_{c}^{+}\right)=\Gamma^{\operatorname{dec}}\left(\Lambda_{c}^{+}\right)+\cos \theta_{C}^{2} \Gamma^{\mathrm{ann}}+\Gamma_{-}^{\mathrm{int}}+\sin \theta_{C}^{2} \Gamma_{+}^{\mathrm{int}}, \\
& \Gamma_{\mathrm{NL}}\left(\Xi_{c}^{+}\right)=\Gamma^{\operatorname{dec}}\left(\Xi_{c}^{+}\right)+\sin \theta_{C}^{2} \Gamma^{\mathrm{ann}}+\Gamma_{-}^{\mathrm{int}}+\cos \theta_{C}^{2} \Gamma_{+}^{\mathrm{int}}, \\
& \Gamma_{\mathrm{NL}}\left(\Xi_{c}^{0}\right)=\Gamma^{\operatorname{dec}}\left(\Xi_{c}^{0}\right)+\Gamma^{\mathrm{ann}}+\Gamma_{+}^{\mathrm{int}}, \\
& \Gamma_{\mathrm{NL}}\left(\Omega_{c}^{0}\right)=\Gamma^{\operatorname{dec}}\left(\Omega_{c}^{0}\right)+6 \sin \theta_{C}^{2} \Gamma^{\mathrm{ann}}+\frac{10}{3} \cos \theta_{C}^{2} \Gamma_{+}^{\mathrm{int}},
\end{aligned}
$$

where $\theta_{C}$ is the Cabibbo angle.

The results of a model calculation in Ref. [86] are shown in Table 10. The lifetime pattern

$$
\tau\left(\Xi_{c}^{+}\right)>\tau\left(\Lambda_{c}^{+}\right)>\tau\left(\Xi_{c}^{0}\right)>\tau\left(\Omega_{c}^{0}\right)
$$

clearly agrees with experiment. This lifetime hierarchy is qualitatively understandable. The $\Xi_{c}^{+}$baryon is the longest-lived among charmed baryons because of the smallness of $W$-exchange and partial cancellation between constructive and destructive Pauli interference, whereas $\Omega_{c}$ is the shortest-lived owing to the presence of two $s$ quarks in the $\Omega_{c}$, which greatly enhances the contribution of $\Gamma_{+}^{\mathrm{int}}$. Because $\Gamma_{+}^{\mathrm{int}}$ is always positive, $\Gamma_{-}^{\mathrm{int}}$ is negative, and the constructive interference has a larger magnitude than the destructive interference, this explains why $\tau\left(\Xi_{c}^{+}\right)>\tau\left(\Lambda_{c}^{+}\right)$. It is also clear from Table 10 that, although the qualitative feature of the lifetime pattern is comprehensive, the quantitative estimates of charmed baryon lifetimes and their ratios are still rather poor.

\subsection{Doubly charmed baryons}

The inclusive nonleptonic rates of doubly charmed baryons in the valence quark approximation and in the 
Table 11 Predicted lifetimes of doubly charmed baryons in units of $10^{-13} \mathrm{~s}$.

\begin{tabular}{|c|c|c|c|c|}
\hline & $\begin{array}{c}\text { Kiselev et al. } \\
{[87,88]}\end{array}$ & $\begin{array}{r}\text { Guberina } \\
{[89]}\end{array}$ & $\begin{array}{l}\operatorname{lng} e \\
{[90]}\end{array}$ & $\begin{array}{c}\text { iner } \\
{[52]}\end{array}$ \\
\hline$\overline{\Xi_{c c}^{++}}$ & $4.6 \pm 0.5$ & 10.5 & 6.7 & 1.85 \\
\hline$\Xi_{c c}^{+}$ & $1.6 \pm 0.5$ & 2.0 & 2.5 & 0.53 \\
\hline$\Omega_{c c}^{+}$ & $2.7 \pm 0.6$ & 3.0 & 2.1 & \\
\hline
\end{tabular}

limit $m_{s} / m_{c}=0$ are expressed as

$$
\begin{aligned}
& \Gamma_{\mathrm{NL}}\left(\Xi_{c c}^{+}\right)=\Gamma^{\operatorname{dec}}\left(\Xi_{c c}^{+}\right)+\cos \theta_{C}^{2} \Gamma^{\mathrm{ann}}+\sin \theta_{C}^{2} \Gamma_{+}^{\mathrm{int}}, \\
& \Gamma_{\mathrm{NL}}\left(\Xi_{c c}^{++}\right)=\Gamma^{\operatorname{dec}}\left(\Xi_{c c}^{++}\right)+\Gamma_{-}^{\mathrm{int}}, \\
& \Gamma_{\mathrm{NL}}\left(\Omega_{c c}^{+}\right)=\Gamma^{\operatorname{dec}}\left(\Omega_{c c}^{+}\right)+\sin \theta_{C}^{2} \Gamma^{\mathrm{ann}}+\cos \theta_{C}^{2} \Gamma_{+}^{\mathrm{int}} .
\end{aligned}
$$

Because $\Gamma_{+}^{\text {int }}$ is positive and $\Gamma_{-}^{\text {int }}$ is negative, it is obvious that $\Xi_{c c}^{++}$is longest-lived, whereas $\Xi_{c c}^{+}\left(\Omega_{c c}^{+}\right)$is the shortest-lived if $\Gamma_{+}^{\text {int }}>\Gamma^{\text {ann }}\left(\Gamma_{+}^{\text {int }}<\Gamma^{\text {ann }}\right)$. In general, we have

$$
\tau\left(\Xi_{c c}^{++}\right) \gg \tau\left(\Omega_{c c}^{+}\right) \sim \tau\left(\Xi_{c c}^{+}\right) .
$$

The predictions available in the literature are summarized in Table 11. Note that the lifetime of $\Xi_{c c}^{+}$was measured by SELEX to be $\tau\left(\Xi_{c c}^{+}\right)<0.33 \times 10^{-13} \mathrm{~s}[46]$.

Because the mass splitting between $\Xi_{c c}^{*}$ and $\Xi_{c c}$ and between $\Omega_{c c}^{*}$ and $\Omega_{c c}$ is less than $100 \mathrm{MeV}$ (see also Eq. (2.6) for the lattice calculations),

$$
\begin{aligned}
& m_{\Xi_{c c}^{*}}-m_{\Xi_{c c}}=m_{\Sigma_{c}^{*}}-m_{\Sigma_{c}} \approx 65 \mathrm{MeV}, \\
& m_{\Omega_{c c}^{*}}-m_{\Omega_{c c}}=m_{\Omega_{c}^{*}}-m_{\Omega_{c}} \approx 71 \mathrm{MeV},
\end{aligned}
$$

it is clear that only electromagnetic decays are allowed for $\Omega_{c c}^{*}$ and $\Xi_{c c}^{*}$.

\section{Hadronic weak decays}

\subsection{Nonleptonic decays}

In contrast to the significant advances made over the last 10 years or so in the study of hadronic weak decays in the bottom baryon sector, progress in the arena of charmed baryons, both theoretical and experimental, has been very slow.

In the naive factorization approach, the coefficients $a_{1}$ for the external $W$-emission amplitude and $a_{2}$ for internal $W$-emission are given by $\left(c_{1}+\frac{c_{2}}{N_{c}}\right)$ and $\left(c_{2}+\frac{c_{1}}{N_{c}}\right)$, respectively. However, we have learned from charmed meson decays that the naive factorization approach never works for the decay rate of color-suppressed decay modes, although it usually works for color-allowed decays. Empirically, it was learned in the 1980s that if the Fierz-transformed terms characterized by $1 / N_{c}$ are dropped, the discrepancy between theory and experiment is greatly reduced [91-93]. This leads to the socalled large- $N_{c}$ approach to describing hadronic $D$ decays [94]. Theoretically, explicit calculations based on QCD sum-rule analysis [95-97] indicate that the Fierz terms are indeed largely compensated by the nonfactorizable corrections.

As the $1 / N_{c}$ expansion method greatly reduces the discrepancy between theory and experiment for charmed meson decays, it is natural to ask if this scenario also works in the baryon sector. This issue can be settled by experimental measurement of the Cabibbo-suppressed mode $\Lambda_{c}^{+} \rightarrow p \phi$, which receives contributions only from the factorizable diagrams. As pointed out in Ref. [98], the rate predicted by the large- $N_{c}$ approach is in good agreement with the measured value. In contrast, its decay rate predicted by the naive factorization approximation will be too small by a factor of 15 . Therefore, the $1 / N_{c}$ approach also works for the factorizable amplitude of charmed baryon decays. This also implies that the inclusion of nonfactorizable contributions is inevitable and necessary. If nonfactorizable effects amount to a redefinition of the effective parameters $a_{1}, a_{2}$, and are universal (i.e., channel-independent) in charm decays, then we still have a new factorization scheme with the universal parameters $a_{1}, a_{2}$ to be determined from experiment.

It is known that for heavy mesons, the nonfactorizable contributions will render color suppression of internal $W$-emission ineffective. However, $W$-exchange in baryon decays is not subject to color suppression even in the absence of nonfactorizable terms. A simple way to see this is to consider the large- $N_{c}$ limit. Although the $W$-exchange diagram is down by a factor of $1 / N_{c}$ relative to the external $W$-emission one, this difference is compensated by the fact that the baryon contains $N_{c}$ quarks in the limit of large $N_{c}$, thus allowing $N_{c}$ different possibilities for $W$ exchange between heavy and light quarks [100]. That is, the pole contribution can be as important as the factorizable one. Experimental measurement of the decay modes $\Lambda_{c}^{+} \rightarrow \Xi^{0} K^{+}, \Delta^{++} K^{-}$, which proceed only through the $W$-exchange contributions, indicates that $W$-exchange indeed plays an essential role in charmed baryon decays.

Various theoretical approaches to weak decays of heavy baryons have been investigated, including the current algebra approach [101-117], factorization scheme, pole model technique [98, 118-122], relativistic quark model [100, 123], and quark diagram scheme [124, 125]. Model predictions of the branching fractions and decay asymmetries can be found in Tables VI-VII of [1] for $\mathcal{B}_{c} \rightarrow \mathcal{B}+P$ decays, Table VIII for $\mathcal{B}_{c} \rightarrow \mathcal{B}+V$ decays and Table IX for $\mathcal{B}_{c} \rightarrow \mathcal{B}\left(\frac{3}{2}^{+}\right)+P(V)$ decays. 
Table 12 Branching fractions of the Cabibbo-allowed two-body decays of $\Lambda_{c}^{+}$in units of \%. Data are taken from PDG [8] except that the absolute branching fraction $\mathcal{B}\left(\Lambda_{c}^{+} \rightarrow p K^{-} \pi^{+}\right)=(5.0 \pm 1.3) \%$ is replaced by the new measurement of $\left(6.84 \pm 0.24_{-0.27}^{+0.21}\right) \%$ by Belle $[126]$.

\begin{tabular}{cc||cc||cc}
\hline Decay & $\mathcal{B}$ & Decay & $\mathcal{B}$ & Decay & $\mathcal{B}$ \\
\hline$\Lambda_{c}^{+} \rightarrow \Lambda \pi^{+}$ & $1.46 \pm 0.13$ & $\Lambda_{c}^{+} \rightarrow \Lambda \rho^{+}$ & $<6.5$ & $\Lambda_{c}^{+} \rightarrow \Delta^{++} K^{-}$ & $1.16 \pm 0.07$ \\
$\Lambda_{c}^{+} \rightarrow \Sigma^{0} \pi^{+}$ & $1.44 \pm 0.14$ & $\Lambda_{c}^{+} \rightarrow \Sigma^{0} \rho^{+}$ & & $\Lambda_{c}^{+} \rightarrow \Sigma^{* 0} \pi^{+}$ & \\
$\Lambda_{c}^{+} \rightarrow \Sigma^{+} \pi^{0}$ & $1.37 \pm 0.30$ & $\Lambda_{c}^{+} \rightarrow \Sigma^{+} \rho^{0}$ & $<1.9$ & $\Lambda_{c}^{+} \rightarrow \Sigma^{*+} \pi^{0}$ & \\
$\Lambda_{c}^{+} \rightarrow \Sigma^{+} \eta$ & $0.75 \pm 0.11$ & $\Lambda_{c}^{+} \rightarrow \Sigma^{+} \omega$ & $3.7 \pm 1.0$ & $\Lambda_{c}^{+} \rightarrow \Sigma^{*+} \eta$ & $1.16 \pm 0.35$ \\
$\Lambda_{c}^{+} \rightarrow \Sigma^{+} \eta^{\prime}$ & & $\Lambda_{c}^{+} \rightarrow \Sigma^{+} \phi$ & $0.42 \pm 0.07$ & $\Lambda_{c}^{+} \rightarrow \Sigma^{*+} \eta^{\prime}$ & \\
$\Lambda_{c}^{+} \rightarrow \Xi^{0} K^{+}$ & $0.53 \pm 0.13$ & $\Lambda_{c}^{+} \rightarrow \Xi^{0} K^{*+}$ & $0.53 \pm 0.19$ & $\Lambda_{c}^{+} \rightarrow \Xi^{* 0} K^{+}$ & $0.36 \pm 0.10$ \\
$\Lambda_{c}^{+} \rightarrow p \bar{K}^{0}$ & $3.2 \pm 0.3$ & $\Lambda_{c}^{+} \rightarrow p \bar{K}^{* 0}$ & $2.1 \pm 0.3$ & $\Lambda_{c}^{+} \rightarrow \Delta^{+} \bar{K}^{0}$ & $1.36 \pm 0.44$ \\
\hline
\end{tabular}

\subsection{Discussion}

\subsection{1 $\Lambda_{c}^{+}$decays}

Experimentally, nearly all the branching fractions of the $\Lambda_{c}^{+}$are measured relative to the $p K^{-} \pi^{+}$mode. On the basis of ARGUS and CLEO data, the PDG has made a model-dependent determination of the absolute branching fraction, $\mathcal{B}\left(\Lambda_{c}^{+} \rightarrow p K^{-} \pi^{+}\right)=(5.0 \pm 1.3) \%$ [8]. Recently, Belle reported a value of $\left(6.84 \pm 0.24_{-0.27}^{+0.21}\right) \%$ [126] from the reconstruction of $D^{*} p \pi$ recoiling against the $\Lambda_{c}^{+}$ production in $e^{+} e^{-}$annihilation. Hence, the uncertainties are much smaller, and, most importantly, this measurement is model independent! More recently, BESIII has also measured this mode directly with the preliminary result $\mathcal{B}\left(\Lambda_{c}^{+} \rightarrow p K^{-} \pi^{+}\right)=(5.84 \pm 0.27 \pm 0.23) \%$ [127]. Its precision is comparable to that of Belle's result. Another approach is to exploit a particular decay, $B^{+} \rightarrow p \pi^{+} \pi^{+} \bar{\Sigma}_{c}^{--}$, and its charge conjugate to measure $\mathcal{B}\left(\Lambda_{c}^{+} \rightarrow p K^{-} \pi^{+}\right)$, also in a model independent manner [128].

The branching fractions of the Cabibbo-allowed twobody decays of $\Lambda_{c}^{+}$are listed in Table 12. The central values of data taken from the PDG [8] are scaled up by a factor of 1.37 because of the new measurement of $\mathcal{B}\left(\Lambda_{c}^{+} \rightarrow p K^{-} \pi^{+}\right)$by Belle [126]. BESIII recently measured 2-body, 3-body, and 4-body decay modes of $\Lambda_{c}^{+}$ with significantly improved precision [127]. For example, the result $\mathcal{B}\left(\Lambda_{c}^{+} \rightarrow \Lambda \pi^{+}\right)=(1.24 \pm 0.07 \pm 0.03) \%$ obtained by BESIII has much better precision than the value of $(1.07 \pm 0.28) \%$ quoted by the PDG [8].

Many of the $\Lambda_{c}^{+}$decay modes such as $\Sigma^{+} K^{+} K^{-}, \Sigma^{+} \phi$, $\Xi^{(*)} K^{(*)+}$, and $\Delta^{++} K^{-}$can proceed only through $W$ exchange. Experimental measurement of them implies the importance of $W$-exchange, which is not subject to color suppression in charmed baryon decays.

Some Cabibbo-suppressed modes such as $\Lambda_{c}^{+} \rightarrow \Lambda K^{+}$ and $\Lambda_{c}^{+} \rightarrow \Sigma^{0} K^{+}$have been measured by Belle [129] and
BaBar [130], respectively. Their branching fractions are of the order of $10^{-3}-10^{-4}$. The first measured Cabibbosuppressed mode $\Lambda_{c}^{+} \rightarrow p \phi$, is of particular interest because it receives contributions only from the factorizable diagram and is expected to be color suppressed in the naive factorization approach. A calculation in Refs. [131, 132 ] yields

$$
\begin{aligned}
\mathcal{B}\left(\Lambda_{c}^{+} \rightarrow p \phi\right) & =2.26 \times 10^{-3} a_{2}^{2}, \\
\alpha\left(\Lambda_{c}^{+} \rightarrow p \phi\right) & =-0.10 .
\end{aligned}
$$

From the experimental measurement $\mathcal{B}\left(\Lambda_{c}^{+} \rightarrow p \phi\right)=$ $(11.2 \pm 2.3) \times 10^{-4}[8]^{2)}$, it follows that

$$
\left|a_{2}\right|_{\operatorname{expt}}=0.70 \pm 0.07 \text {. }
$$

This is consistent with the result obtained by the $1 / N_{c}$ approach, $a_{2}=c_{2}\left(m_{c}\right) \approx-0.59$.

All the models except that of Ref. [121] predict a positive decay asymmetry $\alpha$ for the decay $\Lambda_{c}^{+} \rightarrow \Sigma^{+} \pi^{0}$ (see Table VII of Ref. [1]). Therefore, the measurement of $\alpha=-0.45 \pm 0.31 \pm 0.06$ by CLEO [133] is very surprising. If the negative sign of $\alpha$ is confirmed in the future, this will imply that the $s$-wave and $p$-wave amplitudes for this decay have opposite signs, contrary to the model expectation. The implication of this has been discussed in detail in Refs. [98, 99]. Because the error of the previous CLEO measurement is very large, it is crucial to more accurately measure the decay asymmetry for $\Lambda_{c}^{+} \rightarrow \Sigma^{+} \pi^{0}$.

\subsection{2 $\Xi_{c}^{+}$decays}

No absolute branching fractions have been measured. The branching ratios listed in Tables VI and VIII of Ref. [1] are those relative to $\Xi_{c}^{+} \rightarrow \Xi^{-} \pi^{+} \pi^{+}$. Several Cabibbo-suppressed decay modes such as $p \bar{K}^{* 0}, \Sigma^{+} \phi$, $\Sigma^{+} \pi^{+} \pi^{-}, \Sigma^{-} \pi^{+} \pi^{+}$, and $\Xi(1690) K^{+}$have been observed [8].

The Cabibbo-allowed decays $\Xi_{c}^{+} \rightarrow \mathcal{B}\left(3 / 2^{+}\right)+P$ have been studied and are believed to be forbidden, as they

2) We have scaled up the PDG number $(8.7 \pm 2.7) \times 10^{-4}[8]$ by a factor of 1.37 for its central value. 
do not receive factorizable and $1 / 2^{ \pm}$pole contributions $[100,120]$. However, the $\Sigma^{*+} \bar{K}^{0}$ mode was seen earlier by FOCUS [134], and this may indicate the importance of pole contributions beyond low-lying $1 / 2^{ \pm}$intermediate states.

\subsection{3 $\Xi_{c}^{0}$ decays}

No absolute branching fractions have been measured to date. However, there are several measurements of the ratios of branching fractions, for example [8],

$$
\begin{aligned}
& R_{1}=\frac{\Gamma\left(\Xi_{c}^{0} \rightarrow \Lambda K_{S}^{0}\right)}{\Gamma\left(\Xi_{c}^{0} \rightarrow \Xi^{-} \pi^{+}\right)}=0.21 \pm 0.02 \pm 0.02, \\
& R_{2}=\frac{\Gamma\left(\Xi_{c}^{0} \rightarrow \Omega^{-} K^{+}\right)}{\Gamma\left(\Xi_{c}^{0} \rightarrow \Xi^{-} \pi^{+}\right)}=0.297 \pm 0.024 .
\end{aligned}
$$

The decay modes $\Xi_{c}^{0} \rightarrow \Omega^{-} K^{+}$and $\Sigma^{+} K^{-}$and $\Sigma^{+} \pi^{-}$proceed only through $W$-exchange. The measured branching ratio of $\Omega^{-} K^{+}$relative to $\Xi^{-} \pi^{+}$implies that the $W$-exchange mechanism plays a significant role. The model of Körner and Krämer [100] predicts $R_{2}=0.33$ (see Table IX of Ref. [1]), in agreement with experiment, but its prediction $R_{1}=0.06$ is too small compared to the data.

\subsection{4 $\Omega_{c}^{0}$ decays}

A unique feature of $\Omega_{c}^{0}$ decays is that the decay $\Omega_{c}^{0} \rightarrow$ $\Omega^{-} \pi^{+}$proceeds only via external $W$-emission, whereas $\Omega_{c}^{0} \rightarrow \Xi^{* 0} \bar{K}^{0}$ proceeds via the factorizable internal $W$ emission diagram. Various model predictions of Cabibboallowed $\Omega_{c}^{0} \rightarrow \mathcal{B}\left(3 / 2^{+}\right)+P(V)$ are listed in Table IX of [1] with the unknown parameters $a_{1}$ and $a_{2}$. From the decay $\Lambda_{c}^{+} \rightarrow p \phi$ we learn that $\left|a_{2}\right|=0.70 \pm 0.07$. The hadronic weak decays of the $\Omega_{c}^{0}$ were recently studied in great detail in Ref. [135], where most of the decay channels in $\Omega_{c}^{0}$ decays were found to proceed only through the $W$-exchange diagram; moreover, the $W$-exchange contributions dominate in the rest of the processes, with some exceptions. Observation of such decays would shed light on the mechanism of $W$-exchange effects in these decay modes.

\subsection{Charm-flavor-conserving nonleptonic decays}

There is a special class of weak decays of charmed baryons that can be studied reliably, namely, heavyflavor-conserving nonleptonic decays. Some examples are the singly Cabibbo-suppressed decays $\Xi_{c} \rightarrow \Lambda_{c} \pi$ and $\Omega_{c} \rightarrow \Xi_{c}^{\prime} \pi$. The idea is simple: In these decays, only the light quarks inside the heavy baryon will partici- pate in weak interactions; that is, while the two light quarks undergo weak transitions, the heavy quark behaves as a "spectator". As the emitted light mesons are soft, the $\Delta S=1$ weak interactions among light quarks can be handled by the well known short-distance effective Hamiltonian. This special class of weak decays can usually be calculated more reliably than the conventional charmed baryon weak decays. The synthesis of the heavy quark and chiral symmetries provides a natural setting for investigating these reactions [136]. The weak decays $\Xi_{Q} \rightarrow \Lambda_{Q} \pi$ with $Q=c, b$ were also studied in Refs. [137-139].

The combined symmetries of heavy and light quarks severely restrict the weak interactions allowed. In the symmetry limit, it is found that $\mathcal{B}_{\overline{3}}-\mathcal{B}_{6}$ and $\mathcal{B}_{6}^{*}-\mathcal{B}_{6}$ nonleptonic weak transitions [136] cannot occur. Symmetries alone permit three types of transitions: $\mathcal{B}_{\overline{3}}-\mathcal{B}_{\overline{3}}$, $\mathcal{B}_{6}-\mathcal{B}_{6}$ and $\mathcal{B}_{6}^{*}-\mathcal{B}_{6}$ transitions. However, in both the MIT bag and diquark models, only $\mathcal{B}_{\overline{3}}-\mathcal{B}_{\overline{3}}$ transitions have nonzero amplitudes. The general amplitude for $\mathcal{B}_{i} \rightarrow \mathcal{B}_{f}+P$ is given by

$$
M\left(\mathcal{B}_{i} \rightarrow \mathcal{B}_{f}+P\right)=i \bar{u}_{f}\left(A-B \gamma_{5}\right) u_{i},
$$

where $A$ and $B$ are the $S$ - and $P$-wave amplitudes, respectively. The $S$-wave amplitude can be evaluated using current algebra in terms of the parity-violating commutator term. For example, the $S$-wave amplitude of $\Xi_{c}^{+} \rightarrow \Lambda_{c}^{+} \pi^{0}$ is given by

$$
A\left(\Xi_{c}^{+} \rightarrow \Lambda_{c}^{+} \pi^{0}\right)=-\frac{1}{\sqrt{2} f_{\pi}}\left\langle\Lambda_{c}^{+} \uparrow\left|\mathcal{H}_{\mathrm{eff}}^{\mathrm{PC}}\right| \Xi_{c}^{+} \uparrow\right\rangle,
$$

whereas the $P$-wave amplitude arises from the groundstate baryon poles [136]:

$$
\begin{aligned}
& B\left(\Xi_{c}^{+} \rightarrow \Lambda_{c}^{+} \pi^{0}\right) \\
& \quad=\frac{g_{2}}{2 f_{\pi}} \frac{m_{\Xi_{c}}+m_{\Xi_{c}^{\prime}}}{m_{\Lambda_{c}}-m_{\Xi_{c 2}}}\left\langle\Lambda_{c}^{+} \uparrow\left|\mathcal{H}_{\mathrm{eff}}^{\mathrm{PC}}\right| \Xi_{c}^{+} \uparrow\right\rangle \sin \phi,
\end{aligned}
$$

where $\phi$ is the mixing angle of $\Xi_{c}$, and $\Xi_{c}^{\prime}$ and $\Xi_{c 1}, \Xi_{c 2}$ are their mass eigenstates. The matrix element $\left\langle\Lambda_{c}^{+} \uparrow\right.$ $\left.\left|\mathcal{H}_{\mathrm{eff}}^{\mathrm{PC}}\right| \Xi_{c}^{+} \uparrow\right\rangle$ was evaluated in Ref. [136] using two different models: the MIT bag model [140, 141] and the diquark model.

The predicted rates are [136]

$$
\begin{aligned}
& \Gamma\left(\Xi_{c}^{0} \rightarrow \Lambda_{c}^{+} \pi^{-}\right)=1.7 \times 10^{-15} \mathrm{GeV}, \\
& \Gamma\left(\Xi_{c}^{+} \rightarrow \Lambda_{c}^{+} \pi^{0}\right)=1.0 \times 10^{-15} \mathrm{GeV}, \\
& \Gamma\left(\Omega_{c}^{0} \rightarrow \Xi_{c}^{\prime+} \pi^{-}\right)=4.3 \times 10^{-17} \mathrm{GeV},
\end{aligned}
$$

and the corresponding branching fractions are

$$
\begin{aligned}
& \mathcal{B}\left(\Xi_{c}^{0} \rightarrow \Lambda_{c}^{+} \pi^{-}\right)=2.9 \times 10^{-4}, \\
& \mathcal{B}\left(\Xi_{c}^{+} \rightarrow \Lambda_{c}^{+} \pi^{0}\right)=6.7 \times 10^{-4},
\end{aligned}
$$




$$
\mathcal{B}\left(\Omega_{c}^{0} \rightarrow \Xi_{c}^{\prime+} \pi^{-}\right)=4.5 \times 10^{-6} .
$$

As stated above, the $\mathcal{B}_{6}-\mathcal{B}_{6}$ transition $\Omega_{c}^{0} \rightarrow \Xi_{c}^{\prime+} \pi^{-}$ vanishes in the chiral limit. It receives a finite factorizable contribution as a result of a symmetry-breaking effect. At any rate, the predicted branching fractions for the charm-flavor-conserving decays $\Xi_{c}^{0} \rightarrow \Lambda_{c}^{+} \pi^{-}$and $\Xi_{c}^{+} \rightarrow \Lambda_{c}^{+} \pi^{0}$ are of the order of $10^{-3}-10^{-4}$ and should be readily accessible in the near future.

\subsection{Semileptonic decays}

Exclusive semileptonic decays of charmed baryons: $\Lambda_{c}^{+} \rightarrow \Lambda e^{+}\left(\mu^{+}\right) \nu_{e}, \Xi_{c}^{+} \rightarrow \Xi^{0} e^{+} \nu_{e}$ and $\Xi_{c}^{0} \rightarrow \Xi^{-} e^{+} \nu_{e}$ have been observed experimentally. Their rates depend on the $\mathcal{B}_{c} \rightarrow \mathcal{B}$ form factors $f_{i}\left(q^{2}\right)$ and $g_{i}\left(q^{2}\right)(i=1,2,3)$, which are defined as

$$
\begin{aligned}
& \left\langle\mathcal{B}_{f}\left(p_{f}\right)\left|V_{\mu}-A_{\mu}\right| \mathcal{B}_{c}\left(p_{i}\right)\right\rangle \\
& =\bar{u}_{f}\left(p_{f}\right)\left[f_{1}\left(q^{2}\right) \gamma_{\mu}+i f_{2}\left(q^{2}\right) \sigma_{\mu \nu} q^{\nu}+f_{3}\left(q^{2}\right) q_{\mu}\right. \\
& \left.-\left(g_{1}\left(q^{2}\right) \gamma_{\mu}+i g_{2}\left(q^{2}\right) \sigma_{\mu \nu} q^{\nu}+g_{3}\left(q^{2}\right) q_{\mu}\right) \gamma_{5}\right] u_{i}\left(p_{i}\right) .
\end{aligned}
$$

These form factors have been evaluated using the nonrelativistic quark model [131, 132, 142-144, 151], MIT bag model [142, 143], relativistic quark model [145, 149], light-front quark model [146], and QCD sum rules [147, 148, 150]. Experimentally, the only information available to date is the form-factor ratio measured in the semileptonic decay $\Lambda_{c} \rightarrow \Lambda e \bar{\nu}$. In the heavy quark limit, the six $\Lambda_{c} \rightarrow \Lambda$ form factors are reduced to two:

$$
\begin{aligned}
& \left\langle\Lambda(p)\left|\bar{s} \gamma_{\mu}\left(1-\gamma_{5}\right) c\right| \Lambda_{c}(v)\right\rangle=\bar{u}_{\Lambda}\left(F_{1}^{\Lambda_{c} \Lambda}(v \cdot p)\right. \\
& \left.+\psi F_{2}^{\Lambda_{c} \Lambda}(v \cdot p)\right) \gamma_{\mu}\left(1-\gamma_{5}\right) u_{\Lambda_{c}} .
\end{aligned}
$$

Assuming that the form factors exhibit dipole $q^{2}$ behavior, the ratio $R=\tilde{F}_{2}^{\Lambda_{c} \Lambda} / \tilde{F}_{1}^{\Lambda_{c} \Lambda}$ is measured by CLEO to be $[152]$

$$
R=-0.31 \pm 0.05 \pm 0.04 .
$$

Various model predictions of the charmed baryon semileptonic decay rates and decay asymmetries are shown in Table 13. Dipole $q^{2}$ dependence of the form factors is assumed whenever the form factor momentum dependence is not available in the model. Four different sets of predictions for $\Lambda_{c}^{+} \rightarrow n e^{+} \nu_{e}$, which are not listed in Table 13, were presented in the sum rule calculations of Ref. [153]. The semileptonic decays of $\Omega_{c}$ were treated in Ref. [154] within the framework of a constituent quark model. From Table 13, we see that the computed branching fractions of $\Lambda_{c}^{+} \rightarrow \Lambda e^{+} \nu$, which fall in the range $1.4 \% \sim 2.6 \%$ are slightly smaller than the experimental values, $(2.9 \pm 0.5) \%[(2.1 \pm 0.6) \%$, of the PDG [8]]. The branching fractions of $\Xi_{c}^{0} \rightarrow \Xi^{-} e^{+} \nu$ and $\Xi_{c}^{+} \rightarrow \Xi^{0} e^{+} \nu$ are predicted to lie in the ranges $(0.8-2.0) \%$ and $(3.3-8.1) \%$, respectively, except that the QCD sum rule calculation in Ref. [150] predicts a much larger rate for $\Xi_{c} \rightarrow \Xi e^{+} \nu_{e}$. Experimentally, only the ratios of the branching fractions are available to date [8]:

$$
\begin{aligned}
& \frac{\Gamma\left(\Xi_{c}^{+} \rightarrow \Xi^{0} e^{+} \nu\right)}{\Gamma\left(\Xi_{c}^{+} \rightarrow \Xi^{-} \pi^{+} \pi^{+}\right)}=2.3 \pm 0.6_{-0.6}^{+0.3}, \\
& \frac{\Gamma\left(\Xi_{c}^{0} \rightarrow \Xi^{-} e^{+} \nu\right)}{\Gamma\left(\Xi_{c}^{0} \rightarrow \Xi^{-} \pi^{+}\right)}=3.1 \pm 1.0_{-0.5}^{+0.3} .
\end{aligned}
$$

There have been active studies of semileptonic decays of doubly charmed baryons. The interested reader can consult [155-159] for further references.

Just as with the hadronic decays discussed in the last subsection, there are also heavy-flavor-conserving semileptonic processes, for example, $\Xi_{c}^{0} \rightarrow \Lambda_{c}^{+}\left(\Sigma_{c}^{+}\right) e^{-} \bar{\nu}_{e}$ and $\Omega_{c}^{0} \rightarrow \Xi_{c}^{+} e^{-} \bar{\nu}_{e}$. In these decays only the light quarks inside the heavy baryon will participate in weak interactions, while the heavy quark behaves as a spectator. This topic was recently investigated in Ref. [139]. Owing to the severe phase-space suppression, the branching fractions are of order $10^{-6}$ in the best cases, and typically $10^{-7}$ to $10^{-8}$.

\section{Electromagnetic and weak radiative decays}

Although radiative decays are well measured in the charmed meson sector, e.g., $D^{*} \rightarrow D \gamma$ and $D_{s}^{*+} \rightarrow D_{s}^{+} \gamma$, only three of the radiative modes in the charmed baryon sector have been seen, namely, $\Xi_{c}^{\prime 0} \rightarrow \Xi_{c}^{0} \gamma, \Xi_{c}^{\prime+} \rightarrow$ $\Xi_{c}^{+} \gamma$ and $\Omega_{c}^{* 0} \rightarrow \Omega_{c}^{0} \gamma$. This is understandable because $m_{\Xi_{c}^{\prime}}-m_{\Xi_{c}} \approx 108 \mathrm{MeV}$, and $m_{\Omega_{c}^{*}}-m_{\Omega_{c}} \approx 71 \mathrm{MeV}$. Hence, $\Xi_{c}^{\prime}$ and $\Omega_{c}^{*}$ are governed by the electromagnetic decays. However, it will be difficult to measure the rates of these decays because these states are too narrow to be experimentally resolvable. Nevertheless, we shall systematically study the two-body electromagnetic decays of charmed baryons and also weak radiative decays.

\subsection{Electromagnetic decays}

In the charmed baryon sector, the following two-body electromagnetic decays are of interest:

$$
\begin{array}{ll}
\mathcal{B}_{6} \rightarrow \mathcal{B}_{\overline{3}}+\gamma: \Sigma_{c} \rightarrow \Lambda_{c}+\gamma, & \Xi_{c}^{\prime} \rightarrow \Xi_{c}+\gamma, \\
\mathcal{B}_{6}^{*} \rightarrow \mathcal{B}_{\overline{3}}+\gamma: \Sigma_{c}^{*} \rightarrow \Lambda_{c}+\gamma, & \Xi_{c}^{\prime *} \rightarrow \Xi_{c}+\gamma, \\
\mathcal{B}_{6}^{*} \rightarrow \mathcal{B}_{6}+\gamma: \Sigma_{c}^{*} \rightarrow \Sigma_{c}+\gamma, & \Xi_{c}^{\prime *} \rightarrow \Xi_{c}^{\prime}+\gamma, \\
\Omega_{c}^{*} \rightarrow \Omega_{c}+\gamma, &
\end{array}
$$

where we denote the spin $\frac{1}{2}$ baryons as $\mathcal{B}_{6}$ and $\mathcal{B}_{\overline{3}}$ for 
Table 13 Predicted semileptonic decay rates (in units of $10^{10} s^{-1}$ ) and decay asymmetries (second entry) in various models. The absolute branching fraction $\mathcal{B}\left(\Lambda_{c}^{+} \rightarrow p K^{-} \pi^{+}\right)=(5.0 \pm 1.3) \%$ is replaced by the new measurement of $\left(6.84 \pm 0.24_{-0.27}^{+0.21}\right) \%$ by Belle [126] for the data of $\Gamma\left(\Lambda_{c}^{+} \rightarrow \Lambda^{0} \ell^{+} \nu_{\ell}\right)$ taken from the PDG [8]. Predictions of [142, 143] are obtained in the non-relativistic quark model and the MIT bag model (in parentheses).

\begin{tabular}{|c|c|c|c|c|c|c|c|c|c|c|c|}
\hline Process & {$[131,132]$} & {$[142,143]$} & {$[144]$} & [145] & {$[146]$} & {$[147]$} & [148] & [149] & {$[150]$} & {$[151]$} & Expt. [8] \\
\hline$\Lambda_{c}^{+} \rightarrow \Lambda^{0} e^{+} \nu_{e}$ & 7.1 & $11.2(7.7)$ & 9.8 & 7.22 & 7.0 & $13.2 \pm 1.8$ & $10.9 \pm 3.0$ & & & & $14.4 \pm 2.6$ \\
\hline & & & & -0.812 & & -1 & $-0.88 \pm 0.03$ & & & & $-0.86 \pm 0.04$ \\
\hline$\Lambda_{c}^{+} \rightarrow \Lambda^{0} \mu^{+} \nu_{e}$ & 7.1 & $11.2(7.7)$ & 9.8 & 7.22 & 7.0 & $13.2 \pm 1.8$ & $10.9 \pm 3.0$ & & & & $13.3 \pm 2.8$ \\
\hline$\Lambda_{c}^{+} \rightarrow n e^{+} \nu_{e}$ & & & & 1.32 & & & & 1.01 & & $0.96,1.37$ & \\
\hline$\Xi_{c} \rightarrow \Xi e^{+} \nu_{e}$ & 7.4 & $18.1(12.5)$ & 8.5 & 8.16 & 9.7 & & & & $64.8 \pm 22.6$ & & seen \\
\hline$\Xi_{c} \rightarrow \Sigma e^{+} \nu_{e}$ & & & & & & & & & $3.3 \pm 1.7$ & & \\
\hline
\end{tabular}

the symmetric sextet $\mathbf{6}$ and antisymmetric antitriplet $\overline{\mathbf{3}}$, respectively, and the spin $\frac{3}{2}$ baryon by $\mathcal{B}_{6}^{*}$.

An ideal theoretical framework for studying the abovementioned electromagnetic decays is provided by the formalism in which the heavy quark symmetry and the chiral symmetry of light quarks are combined [68-70]. When supplemented by the nonrelativistic quark model, the formalism determines completely the low energy dynamics of heavy hadrons. The electromagnetic interactions of heavy hadrons consist of two distinct contributions: one from gauging electromagnetically the chirally invariant strong interaction Lagrangians for heavy mesons and baryons given in Refs. [68-70], and the other from the anomalous magnetic moment couplings of the heavy particles. The heavy quark symmetry reduces the number of free parameters needed to describe the magnetic couplings to the photon. There are two undetermined parameters for the ground-state heavy baryons. All of these parameters are related simply to the magnetic moments of the light quarks in the nonrelativistic quark model. However, the charmed quark is not particularly heavy $\left(m_{c} \simeq 1.6 \mathrm{GeV}\right)$, and it carries a charge of $\frac{2}{3} e$. Consequently, the contribution from its magnetic moment cannot be neglected. The chiral and electromagnetic gaugeinvariant Lagrangian for heavy baryons can be found in Eqs. (3.8) and (3.9) of Ref. [160], and are denoted by $\mathcal{L}_{B}^{(1)}$ and $\mathcal{L}_{B}^{(2)}$, respectively.

The most general gauge-invariant Lagrangian Eq. (3.9) of Ref. [160] for magnetic transitions of heavy baryons can be recast in terms of superfields $[161,162]$ :

$$
\begin{aligned}
\mathcal{L}_{B}^{(2)} & =-i 3 a_{1} \operatorname{tr}\left(\bar{S}^{\mu} Q F_{\mu \nu} S^{\nu}\right) \\
& +\sqrt{3} a_{2} \epsilon_{\mu \nu \alpha \beta} \operatorname{tr}\left(\bar{S}^{\mu} Q v^{\nu} F^{\alpha \beta} T\right)+\text { h.c. } \\
& +3 a_{1}^{\prime} \operatorname{tr}\left(\bar{S}^{\mu} Q^{\prime} \sigma \cdot F S_{\mu}\right)-\frac{3}{2} a_{1}^{\prime} \operatorname{tr}\left(\bar{T} Q^{\prime} \sigma \cdot F T\right),
\end{aligned}
$$

where $\sigma \cdot F \equiv \sigma_{\mu \nu} F^{\mu \nu}, Q=\operatorname{diag}(2 / 3,-1 / 3,-1 / 3)$ is the charge matrix for the light $u, d$, and $s$ quarks, and $Q^{\prime}=e_{Q}$ is the charge of the heavy quark. In the above equation,

$$
T=\mathcal{B}_{\overline{3}}, \quad S^{\mu}=\mathcal{B}_{6}^{* \mu}-\frac{1}{\sqrt{3}}\left(\gamma^{\mu}+v^{\mu}\right) \gamma_{5} \mathcal{B}_{6} .
$$

It follows that $[161,162]$

$$
\begin{aligned}
& A\left[S_{i j}^{\mu}(v) \rightarrow S_{i j}^{\nu}+\gamma(\varepsilon, k)\right] \\
& =i \frac{3}{2} a_{1} \overline{\mathcal{U}}^{\nu}\left(Q_{i i}+Q_{j j}\right)\left(k_{\nu} \varepsilon_{\mu}-k_{\mu} \varepsilon_{\nu}\right) \mathcal{U}^{\mu}-i 6 a_{1}^{\prime} Q^{\prime} \overline{\mathcal{U}}^{\mu} \not k \notin \mathcal{U}_{\mu}, \\
& A\left[S_{i j}^{\mu}(v) \rightarrow T_{i j}+\gamma(\varepsilon, k)\right] \\
& =-2 \sqrt{3 / 2} a_{2} \epsilon_{\mu \nu \alpha \beta} \bar{u}_{\overline{3}} v^{\nu} k^{\alpha} \varepsilon^{\beta}\left(Q_{i i}-Q_{j j}\right) \mathcal{U}^{\mu}(i<j),
\end{aligned}
$$

where $k_{\mu}$ is the photon 4-momentum, and $\varepsilon_{\mu}$ is the polarization 4-vector. As stressed in Ref. [160], SU(3) breaking effects due to light-quark mass differences can be incorporated by replacing the charge matrix $Q$ with

$$
Q \rightarrow \tilde{Q}=\operatorname{diag}\left(\frac{2}{3},-\frac{\alpha}{3},-\frac{\beta}{3}\right)
$$

where $\alpha=M_{u} / M_{d}$ and $\beta=M_{u} / M_{s}$. To avoid any confusion with the current quark mass $m_{q}$, we have used capital letters to denote the constituent quark masses. In the quark model, the coefficients $a_{1}$ and $a_{2}$ are simply related to the Dirac magnetic moments of light quarks,

$$
a_{1}=-\frac{e}{3} \frac{1}{M_{u}}, \quad a_{2}=\frac{e}{2 \sqrt{6}} \frac{1}{M_{u}},
$$

whereas $a_{1}^{\prime}$ is connected to those of heavy quarks. Explicitly, $a_{1}^{\prime}$ is fixed by heavy quark symmetry as

$$
a_{1}^{\prime}=\frac{e}{12} \frac{1}{M_{Q}} .
$$

Within the framework of HHChPT, the authors of [163] proceeded to construct chiral Lagrangians at the level $\mathcal{O}\left(p^{2}\right)$ and $\mathcal{O}\left(p^{3}\right)$ and then calculated the electromagnetic decay amplitudes of charmed baryons up to $\mathcal{O}\left(p^{3}\right)$. It is not clear whether their $\mathcal{O}\left(p^{2}\right)$ Lagrangian (see Eq. (12) of Ref. [163]), characterized by the four couplings $f_{2}, f_{3}, \tilde{f}_{3}$ and $f_{4}$, is equivalent to the first two terms of the $\mathcal{O}(p)$ Lagrangian given by Eq. (6.2). The 
unknown couplings there were also estimated using the quark model.

The general amplitudes of electromagnetic decays are given by [160]

$$
\begin{aligned}
& A\left(\mathcal{B}_{6} \rightarrow \mathcal{B}_{\overline{3}}+\gamma\right)=i \eta_{1} \bar{u}_{\overline{3}} \sigma_{\mu \nu} k^{\mu} \varepsilon^{\nu} u_{6}, \\
& A\left(\mathcal{B}_{6}^{*} \rightarrow \mathcal{B}_{\overline{3}}+\gamma\right)=i \eta_{2} \epsilon_{\mu \nu \alpha \beta} \bar{u}_{\overline{3}} \gamma^{\nu} k^{\alpha} \varepsilon^{\beta} u^{\mu}, \\
& A\left(\mathcal{B}_{6}^{*} \rightarrow \mathcal{B}_{6}+\gamma\right)=i \eta_{3} \epsilon_{\mu \nu \alpha \beta} \bar{u}_{6} \gamma^{\nu} k^{\alpha} \varepsilon^{\beta} u^{\mu} .
\end{aligned}
$$

The corresponding decay rates are [160]

$$
\begin{aligned}
& \Gamma\left(\mathcal{B}_{6} \rightarrow \mathcal{B}_{\overline{3}}+\gamma\right)=\eta_{1}^{2} \frac{k^{3}}{\pi}, \\
& \Gamma\left(\mathcal{B}_{6}^{*} \rightarrow \mathcal{B}_{\overline{3}}+\gamma\right)=\eta_{2}^{2} \frac{k^{3}}{3 \pi} \frac{3 m_{i}^{2}+m_{f}^{2}}{4 m_{i}^{2}}, \\
& \Gamma\left(\mathcal{B}_{6}^{*} \rightarrow \mathcal{B}_{6}+\gamma\right)=\eta_{3}^{2} \frac{k^{3}}{3 \pi} \frac{3 m_{i}^{2}+m_{f}^{2}}{4 m_{i}^{2}},
\end{aligned}
$$

where $m_{i}\left(m_{f}\right)$ is the mass of the parent (daughter) baryon. The coupling constants $\eta_{i}$ can be calculated using the quark model for $a_{1}, a_{2}$, and $a_{1}^{\prime}[160,164]$ :

$$
\begin{aligned}
& \eta_{1}\left(\Sigma_{c}^{+} \rightarrow \Lambda_{c}^{+}\right)=\frac{e}{6 \sqrt{3}}\left(\frac{2}{M_{u}}+\frac{1}{M_{d}}\right), \\
& \eta_{1}\left(\Xi_{c}^{\prime+} \rightarrow \Xi_{c}^{+}\right)=\frac{e}{6 \sqrt{3}}\left(\frac{2}{M_{u}}+\frac{1}{M_{s}}\right), \\
& \eta_{1}\left(\Xi_{c}^{\prime 0} \rightarrow \Xi_{c}^{0}\right)=\frac{e}{6 \sqrt{3}}\left(\frac{1}{M_{s}}-\frac{1}{M_{d}}\right), \\
& \eta_{2}\left(\Sigma_{c}^{*+} \rightarrow \Lambda_{c}^{+}\right)=\frac{e}{3 \sqrt{6}}\left(\frac{2}{M_{u}}+\frac{1}{M_{d}}\right), \\
& \eta_{2}\left(\Xi_{c}^{\prime *+} \rightarrow \Xi_{c}^{+}\right)=\frac{e}{3 \sqrt{6}}\left(\frac{2}{M_{u}}+\frac{1}{M_{s}}\right), \\
& \eta_{2}\left(\Xi_{c}^{\prime * 0} \rightarrow \Xi_{c}^{0}\right)=\frac{e}{3 \sqrt{6}}\left(-\frac{1}{M_{d}}+\frac{1}{M_{s}}\right), \\
& \eta_{3}\left(\Sigma_{c}^{*++} \rightarrow \Sigma_{c}^{++}\right)=\frac{2 \sqrt{2} e}{9}\left(\frac{1}{M_{u}}-\frac{1}{M_{c}}\right), \\
& \eta_{3}\left(\Sigma_{c}^{* 0} \rightarrow \Sigma_{c}^{0}\right)=\frac{2 \sqrt{2} e}{9}\left(-\frac{1}{2 M_{d}}-\frac{1}{M_{c}}\right), \\
& \eta_{3}\left(\Sigma_{c}^{*+} \rightarrow \Sigma_{c}^{+}\right)=\frac{\sqrt{2} e}{9}\left(\frac{1}{M_{u}}-\frac{1}{2 M_{d}}-\frac{2}{M_{c}}\right), \\
& \eta_{3}\left(\Omega_{c}^{* 0} \rightarrow \Omega_{c}^{0}\right)=\frac{2 \sqrt{2} e}{9}\left(-\frac{1}{2 M_{s}}-\frac{1}{M_{c}}\right), \\
& \eta_{3}\left(\Xi_{c}^{*+} \rightarrow \Xi_{c}^{\prime+}\right)=\frac{\sqrt{2} e}{9}\left(\frac{1}{M_{u}}-\frac{1}{2 M_{s}}-\frac{2}{M_{c}}\right), \\
& \eta_{3}\left(\Xi_{c}^{* 0} \rightarrow \Xi_{c}^{\prime 0}\right)=\frac{\sqrt{2} e}{9}\left(-\frac{1}{2 M_{d}}-\frac{1}{2 M_{s}}-\frac{2}{M_{c}}\right) .
\end{aligned}
$$

The results calculated using the constituent quark masses, $M_{u}=338 \mathrm{MeV}, M_{d}=322 \mathrm{MeV}, M_{s}=510$ $\mathrm{MeV}$ [8], and $M_{c}=1.6 \mathrm{GeV}$, are summarized in the second column of Table 14. Some other model predictions are also listed there for comparison.

Radiative decays of $s$-wave charmed baryons are considered in Ref. [165] in the quark model, and the predictions are similar to ours. A similar procedure is followed in Ref. [166] where the heavy quark symmetry is supplemented with light-diquark symmetries to calculate the widths of $\Sigma_{c}^{+} \rightarrow \Lambda_{c}^{+} \gamma$ and $\Sigma_{c}^{*} \rightarrow \Sigma_{c} \gamma$. The authors of Ref. [75] apply the relativistic quark model to predict various electromagnetic decays of charmed baryons. In addition to the magnetic dipole (M1) transition, the author of Ref. [167] also considered and estimated the electric quadrupole (E2) amplitude for $\Sigma_{c}^{*+} \rightarrow \Lambda_{c}^{+} \gamma$ arising from the chiral loop correction. The E2 contributions were analyzed in detail in Ref. [168]. The E2 amplitudes appear at different higher orders for the three types of decays: $\mathcal{O}\left(1 / \Lambda_{\chi}^{2}\right)$ for $\mathcal{B}_{6}^{*} \rightarrow \mathcal{B}_{6}+\gamma, \mathcal{O}\left(1 / m_{Q} \Lambda_{\chi}^{2}\right)$ for $\mathcal{B}_{6}^{*} \rightarrow \mathcal{B}_{\overline{3}}+\gamma$ and $\mathcal{O}\left(1 / m_{Q}^{3} \Lambda_{\chi}^{2}\right)$ for $\mathcal{B}_{6} \rightarrow \mathcal{B}_{\overline{3}}+\gamma$. Therefore, the E2 contribution to $\mathcal{B}_{6} \rightarrow \mathcal{B}_{\overline{3}}+\gamma$ is completely negligible. The electromagnetic decays were calculated in Refs. [169-171] using the QCD sum rule method, and they were studied within the framework of the modified bag model in Ref. [172].

It is evident from Table 14 that the predictions in Refs. $[160,164]$ and $[163]$ all based on HHChPT are quite different for the following three modes: $\Sigma_{c}^{*++} \rightarrow \Sigma_{c}^{++} \gamma$, $\Sigma_{c}^{*+} \rightarrow \Lambda_{c}^{+} \gamma$ and $\Xi_{c}^{\prime *+} \rightarrow \Xi_{c}^{+} \gamma$. Indeed, the results for the last two modes in Ref. [163] are larger than all the other existing predictions by one order of magnitude! It is naively expected that all HHChPT approaches should agree with each other to the lowest order of chiral expansion provided that the coefficients are inferred from the nonrelativistic quark model. The lowest order predictions $\Gamma\left(\Sigma_{c}^{*+} \rightarrow \Lambda_{c}^{+} \gamma\right)=756 \mathrm{keV}$ and $\Gamma\left(\Xi_{c}^{\prime *+} \rightarrow \Xi_{c}^{+} \gamma\right)=403 \mathrm{keV}$ obtained in Ref. [163] are still very large. Note that a recent lattice calculation in Ref. [174] yields $\Gamma\left(\Omega_{c}^{*} \rightarrow \Omega_{c} \gamma\right)=0.074 \pm 0.008 \mathrm{keV}$ which is much smaller than the value of $\Gamma\left(\Omega_{c}^{*}\right)=4.82 \mathrm{keV}$ predicted in Ref. [163].

Chiral loop corrections to the M1 electromagnetic decays and to the strong decays of heavy baryons were computed at the one loop order in Refs. [161, 162]. The leading chiral-loop effects we found are nonanalytic in the forms of $m / \Lambda_{\chi}$ and $\left(m^{2} / \Lambda_{\chi}^{2}\right) \ln \left(\Lambda^{2} / m^{2}\right)$ (or $m_{q}^{1 / 2}$ and $m_{q} \ln m_{q}$, where $m_{q}$ is the light quark mass). Some results are $[161,162]$

$$
\begin{aligned}
& \Gamma\left(\Sigma_{c}^{+} \rightarrow \Lambda_{c}^{+} \gamma\right)=112 \mathrm{keV}, \\
& \Gamma\left(\Xi_{c}^{\prime+} \rightarrow \Xi_{c}^{+} \gamma\right)=29 \mathrm{keV}, \\
& \Gamma\left(\Xi^{\prime}{ }_{c}^{0} \rightarrow \Xi_{c}^{0} \gamma\right)=0.15 \mathrm{keV}
\end{aligned}
$$


Table 14 Electromagnetic decay rates (in units of keV) of $s$-wave charmed baryons. Among the four different results listed in Refs. [165] and [173], we quote those denoted by $\Gamma_{\gamma}^{(0)}$ and "Present (ecqm)", respectively.

\begin{tabular}{|c|c|c|c|c|c|c|c|c|c|c|}
\hline Decay & $\begin{array}{l}\text { HHChPT } \\
{[160,164]}\end{array}$ & $\begin{array}{c}\text { HHChPT } \\
{[163]}\end{array}$ & $\begin{array}{c}\text { Dey } \\
\text { et al. }[165]\end{array}$ & $\begin{array}{c}\text { Ivanov } \\
\text { et al. }[75]\end{array}$ & $\begin{array}{c}\text { Tawfiq } \\
\text { et al. }[166]\end{array}$ & $\begin{array}{c}\text { Bañuls } \\
\text { et al. }[168]\end{array}$ & $\begin{array}{c}\text { Aliev } \\
\text { et al. }[169]\end{array}$ & $\begin{array}{c}\text { Wang } \\
{[170,171]}\end{array}$ & $\begin{array}{l}\text { Bernotas } \\
\text { et al. }[172]\end{array}$ & $\begin{array}{l}\text { Majethiya } \\
\text { et al. }\end{array}$ \\
\hline$\Sigma_{c}^{+} \rightarrow \Lambda_{c}^{+} \gamma$ & 91.5 & 164.16 & 120 & $60.7 \pm 1.5$ & 87 & & & & 46.1 & 60.55 \\
\hline$\Sigma_{c}^{*+} \rightarrow \Lambda_{c}^{+} \gamma$ & 150.3 & 892.97 & 310 & $151 \pm 4$ & & & $130 \pm 45$ & & 126 & 154.48 \\
\hline$\Sigma_{c}^{*+} \rightarrow \Sigma_{c}^{+} \gamma$ & 0.002 & 0.85 & 0.001 & $0.14 \pm 0.004$ & 0.19 & & $0.40 \pm 0.16$ & $0.40_{-0.21}^{+0.43}$ & 0.004 & $<10^{-4}$ \\
\hline$\Sigma_{c}^{* 0} \rightarrow \Sigma_{c}^{0} \gamma$ & 1.2 & 2.92 & 1.2 & & 0.76 & & $0.08 \pm 0.03$ & $1.58_{-0.82}^{+1.68}$ & 1.08 & 1.12 \\
\hline$\Xi_{c}^{\prime *+} \rightarrow \Xi_{c}^{\prime+} \gamma$ & 0.06 & 1.10 & 0.10 & & & & & $0.96_{-0.67}^{+1.47}$ & 0.011 & \\
\hline$\Xi_{c}^{\prime 0} \rightarrow \Xi_{c}^{0} \gamma$ & 0.4 & 0.02 & 0.33 & $0.17 \pm 0.02$ & & $1.2 \pm 0.7$ & & & 0.0015 & \\
\hline$\Xi_{c}^{\prime * 0} \rightarrow \Xi_{c}^{0} \gamma$ & 1.1 & 0.36 & 1.7 & $0.68 \pm 0.04$ & & $5.1 \pm 2.7$ & $0.66 \pm 0.32$ & & 0.908 & 0.30 \\
\hline$\Xi_{c}^{\prime * 0} \rightarrow \Xi_{c}^{\prime 0} \gamma$ & 1.0 & 3.83 & 1.6 & & & & & $1.26_{-0.46}^{+0.80}$ & 1.03 & \\
\hline$\Omega_{c}^{* 0} \rightarrow \Omega_{c}^{0} \gamma$ & 0.9 & 4.82 & 0.71 & & & & & $1.16_{-0.54}^{+1.12}$ & 1.07 & 2.02 \\
\hline
\end{tabular}

Table 15 Electromagnetic decay rates (in units of $\mathrm{keV}$ ) of $p$-wave charmed baryons.

\begin{tabular}{|c|c|c|c|c|c|c|}
\hline Decay & $\begin{array}{c}\text { Ivanov } \\
\text { et al. }[75]\end{array}$ & $\begin{array}{c}\text { Tawfiq } \\
\text { et al. }[166]\end{array}$ & $\begin{array}{c}\text { Aziza Baccouche } \\
\text { et al. }[176]\end{array}$ & $\begin{array}{l}\text { Zhu } \\
{[177]}\end{array}$ & $\begin{array}{l}\text { Chow } \\
{[178]}\end{array}$ & $\begin{array}{c}\text { Gamermann } \\
\text { et al. }[179]\end{array}$ \\
\hline \multicolumn{7}{|l|}{$1 / 2^{-} \rightarrow 1 / 2^{+}\left(3 / 2^{+}\right) \gamma$} \\
\hline$\Lambda_{c}(2595)^{+} \rightarrow \Lambda_{c}^{+} \gamma$ & $115 \pm 1$ & & 25 & 36 & 16 & $274 \pm 52$ \\
\hline$\Lambda_{c}(2595)^{+} \rightarrow \Sigma_{c}^{+} \gamma$ & $77 \pm 1$ & 71 & & 11 & & $2.1 \pm 0.4$ \\
\hline$\Lambda_{c}(2595)^{+} \rightarrow \Sigma_{c}^{*+} \gamma$ & $6 \pm 0.1$ & 11 & & 1 & & \\
\hline \multicolumn{7}{|l|}{$3 / 2^{-} \rightarrow 1 / 2^{+}\left(3 / 2^{+}\right) \gamma$} \\
\hline$\Lambda_{c}(2625)^{+} \rightarrow \Sigma_{c}^{*+} \gamma$ & $46 \pm 0.6$ & 32 & & 6 & & \\
\hline$\Xi_{c}(2815)^{+} \rightarrow \Xi_{c}^{+} \gamma$ & $190 \pm 5$ & & & & & \\
\hline$\Xi_{c}(2815)^{0} \rightarrow \Xi_{c}^{0} \gamma$ & $497 \pm 14$ & & & & & \\
\hline
\end{tabular}

which should be compared with the corresponding quarkmodel results: 92, 20, and $0.4 \mathrm{keV}$, respectively (Table $14)$.

The electromagnetic decays $\Xi_{c}^{\prime * 0} \rightarrow \Xi_{c}^{0} \gamma$ and $\Xi_{c}^{\prime 0} \rightarrow$ $\Xi_{c}^{0} \gamma$ are of special interest. It was advocated in Ref. [175] that a measurement of their branching fractions will allow us to determine one of the coupling constants in HHChPT, namely, $g_{1}$. They are forbidden at tree level in the SU(3) limit [see Eq. (6.10)]. In heavy baryon chiral perturbation theory, this radiative decay is induced via chiral loops where $\mathrm{SU}(3)$ symmetry is broken by the light current quark masses. By identifying the chiral loop contribution to $\Xi_{c}^{\prime * 0} \rightarrow \Xi_{c}^{0} \gamma$ with the quark model prediction given in Eq. (6.10), it was found in Ref. [164] that one of the two possible solutions agrees with the quark model expectation for $g_{1}$.

For the electromagnetic decays of $p$-wave charmed baryons, the search for $\Lambda_{c}(2593)^{+} \rightarrow \Lambda_{c}^{+} \gamma$ and $\Lambda_{c}(2625)^{+} \rightarrow \Lambda_{c}^{+} \gamma$ has not yet succeeded. The interested reader is referred to Refs. $[14,75,166,175-180]$ for more details on the theoretical side. Some predictions are collected in Table 15, and they are more diversified than the $s$-wave case. For electromagnetic decays of doubly charmed baryons, see, e.g., Refs. [172, 181].

The electromagnetic decays considered to date do not critically test the heavy quark symmetry or chiral symmetry. The results follow simply from the quark model. There are examples in which both the heavy quark symmetry and chiral symmetry enter in a crucial way. These are the radiative decays of heavy baryons involving an emitted pion. Some examples that are kinematically allowed are

$$
\Sigma_{c} \rightarrow \Lambda_{c} \pi \gamma, \quad \Sigma_{c}^{*} \rightarrow \Lambda_{c} \pi \gamma, \quad \Sigma_{c}^{*} \rightarrow \Sigma_{c} \pi \gamma, \quad \Xi_{c}^{*} \rightarrow \Xi_{c} \pi \gamma .
$$

The contact interaction dictated by the Lagrangian $\mathcal{L}_{B}^{(1)}$ can be nicely tested by the decay $\Sigma_{c}^{0} \rightarrow \Lambda_{c}^{+} \pi^{-} \gamma$, whereas a test of the chiral structure of $\mathcal{L}_{B}^{(2)}$ is provided by the process $\Sigma_{c}^{+} \rightarrow \Lambda_{c}^{+} \pi^{0} \gamma$; see Ref. [160] for the analysis. 


\subsection{Weak radiative decays}

At the quark level, three different types of processes can contribute to the weak radiative decays of heavy hadrons, namely, single-, two-, and three-quark transitions [183]. The single-quark transition mechanism arises from the so-called electromagnetic penguin diagram. Unfortunately, the penguin process $c \rightarrow u \gamma$ is highly suppressed, so it plays no role in radiative decays of charmed hadrons. There are two contributions from the two-quark transitions: one from the $W$-exchange diagram accompanied by photon emission from the external quark, and the other from the same $W$-exchange diagram but with a photon radiated from the $W$ boson. The latter is typically suppressed by a factor of $m_{q} k / M_{W}^{2}$ (where $k$ is the photon energy) compared to the former bremsstrahlung process [182]. For charmed baryons, the Cabibbo-allowed decay modes via $c \bar{u} \rightarrow s \bar{d} \gamma$ (Fig. 7) or $c d \rightarrow u s \gamma$ are

$$
\Lambda_{c}^{+} \rightarrow \Sigma^{+} \gamma, \quad \Xi_{c}^{0} \rightarrow \Xi^{0} \gamma
$$

Finally, the three-quark transition involving $W$-exchange between two quarks and photon emission by the third quark is quite strongly suppressed because of the very small probability of finding three quarks that are adequately kinematically matched with the baryons $[183$, 184].

The general amplitude of weak radiative decays of baryons is given by

$$
A\left(\mathcal{B}_{i} \rightarrow \mathcal{B}_{f} \gamma\right)=i \bar{u}_{f}\left(a+b \gamma_{5}\right) \sigma_{\mu \nu} \varepsilon^{\mu} k^{\nu} u_{i},
$$

where $a$ and $b$ are the parity-conserving and -violating amplitudes, respectively. The corresponding decay rate is
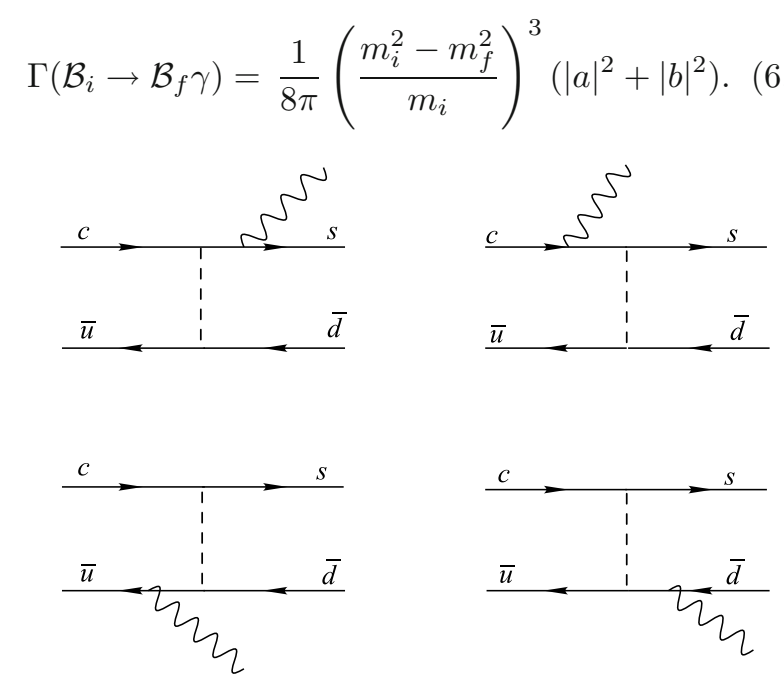

Fig. $7 \quad W$-exchange diagrams contributing to the quark-quark bremsstrahlung process $c+\bar{u} \rightarrow s+\bar{d}+\gamma$. The $W$-annihilation type diagrams are not shown here.
Nonpenguin weak radiative decays of charmed baryons, such as those in Eq. (6.13), are characterized by emission of a hard photon and the presence of a highly virtual intermediate quark between the electromagnetic and weak vertices. It was shown in Ref. [185] that these features should make it possible to analyze these processes by perturbative QCD; that is, these processes can be described by an effective local and gauge invariant Lagrangian:

$$
\mathcal{H}_{\mathrm{eff}}(c \bar{u} \rightarrow s \bar{d} \gamma)=\frac{G_{F}}{2 \sqrt{2}} V_{c s} V_{u d}^{*}\left(c_{+} O_{+}^{F}+c_{-} O_{-}^{F}\right),
$$

with

$$
\begin{aligned}
O_{ \pm}^{F}(c \bar{u} & \rightarrow s \bar{d} \gamma)=\frac{e}{m_{i}^{2}-m_{f}^{2}}\left\{\left(e_{s} \frac{m_{f}}{m_{s}}+e_{u} \frac{m_{i}}{m_{u}}\right)\right. \\
\times & \left(\tilde{F}_{\mu \nu}+i F_{\mu \nu}\right) O_{ \pm}^{\mu \nu}-\left(e_{d} \frac{m_{f}}{m_{d}}+e_{c} \frac{m_{i}}{m_{c}}\right) \\
& \left.\times\left(\tilde{F}_{\mu \nu}-i F_{\mu \nu}\right) O_{\mp}^{\mu \nu}\right\},
\end{aligned}
$$

where $m_{i}=m_{c}+m_{u}, m_{f}=m_{s}+m_{d}, \tilde{F}_{\mu \nu} \equiv \frac{1}{2} \epsilon_{\mu \nu \alpha \beta} F^{\alpha \beta}$ and

$$
\begin{aligned}
O_{ \pm}^{\mu \nu}= & \bar{s} \gamma^{\mu}\left(1-\gamma_{5}\right) c \bar{u} \gamma^{\nu}\left(1-\gamma_{5}\right) d \\
& \pm \bar{s} \gamma^{\mu}\left(1-\gamma_{5}\right) d \bar{u} \gamma^{\nu}\left(1-\gamma_{5}\right) c .
\end{aligned}
$$

For radiative decays of charmed baryons, one needs to evaluate the matrix element $\left\langle\mathcal{B}_{f}\left|O_{ \pm}^{\mu \nu}\right| \mathcal{B}_{i}\right\rangle$. Because the quark-model wave functions most resemble the hadronic states in the frame where both baryons are static, the static MIT bag model was adopted in Ref. [185] for the calculation. The predictions are ${ }^{3)}$

$$
\begin{aligned}
& \mathcal{B}\left(\Lambda_{c}^{+} \rightarrow \Sigma^{+} \gamma\right)=4.9 \times 10^{-5} \\
& \alpha\left(\Lambda_{c}^{+} \rightarrow \Sigma^{+} \gamma\right)=-0.86 \\
& \mathcal{B}\left(\Xi_{c}^{0} \rightarrow \Xi^{0} \gamma\right)=3.5 \times 10^{-5} \\
& \alpha\left(\Xi_{c}^{0} \rightarrow \Xi^{0} \gamma\right)=-0.86 .
\end{aligned}
$$

A different analysis of the same decays in Ref. [186] yielded the following results:

$$
\begin{aligned}
& \mathcal{B}\left(\Lambda_{c}^{+} \rightarrow \Sigma^{+} \gamma\right)=2.8 \times 10^{-4} \\
& \alpha\left(\Lambda_{c}^{+} \rightarrow \Sigma^{+} \gamma\right)=0.02 \\
& \mathcal{B}\left(\Xi_{c}^{0} \rightarrow \Xi^{0} \gamma\right)=1.5 \times 10^{-4} \\
& \alpha\left(\Xi_{c}^{0} \rightarrow \Xi^{0} \gamma\right)=-0.01 .
\end{aligned}
$$

Evidently, these predictions (especially the decay asymmetry) differ greatly from those obtained in Ref. [185].

Finally, it is worth remarking that, analogous to the heavy-flavor-conserving nonleptonic weak decays dis-

\footnotetext{
3) The branching fraction of $\Xi_{c}^{0} \rightarrow \Xi^{0} \gamma$ has been updated using the current lifetime of $\Xi_{c}^{0}$.
} 
cussed in Section 6.3, there is a special class of weak radiative decays in which heavy flavor is conserved, for example, $\Xi_{c} \rightarrow \Lambda_{c} \gamma$ and $\Omega_{c} \rightarrow \Xi_{c} \gamma$. In these decays, weak radiative transitions arise from the light quark sector of the heavy baryon, whereas the heavy quark behaves as a spectator. However, the dynamics of these radiative decays is more complicated than that of their counterpart in nonleptonic weak decays, e.g., $\Xi_{c} \rightarrow \Lambda_{c} \pi$. In any event, it merits an investigation.

\section{Conclusions}

In this report, we began with a brief overview of the spectroscopy of charmed baryons and discussed their possible structure and spin-parity assignments in the quark model. For $p$-wave baryons, we have assigned $\Sigma_{c 2}\left(\frac{3}{2}^{-}\right)$ to $\Sigma_{c}(2800)$. As for first positive-parity excitations, with the help of the relativistic quark-diquark model and the ${ }^{3} P_{0}$ model, we have identified $\tilde{\Lambda}_{c 3}^{2}\left(\frac{5}{2}^{+}\right)$with $\Lambda_{c}(2800)$, $\tilde{\Xi}_{c}^{\prime}\left(\frac{1}{2}^{+}\right)$with $\Xi_{c}(2980)$, and $\tilde{\Xi}_{c 3}^{2}\left(\frac{5}{2}^{+}\right)$with $\Xi_{c}(3080)$, although the first and last assignments may encounter some potential problems.

It should be stressed that mass analysis alone is usually not sufficient to pin down the spin-parity quantum numbers of higher excited charmed baryon states, a study of their strong decays is necessary. For example, $\Sigma_{c 0}\left(\frac{1}{2}^{-}\right)$, $\Sigma_{c 1}\left(\frac{1}{2}^{-}, \frac{3}{2}^{-}\right)$, and $\Sigma_{c 2}\left(\frac{3}{2}^{-}, \frac{5}{2}^{-}\right)$for $\Sigma_{c}(2800)$ all have similar masses. Analysis of strong decays allows us to exclude the first two possibilities. It should be stressed that Regge phenomenology and the mass relations for antitriplet and sextet multiplets also provide very useful guidance for the spin-parity quantum numbers.

On the basis of various theoretical tools such as lattice QCD and the QCD sum rule method, there are many theoretical studies of charmed baryon spectroscopy, especially for doubly and triply charmed baryons. However, progress on the hadronic decays, radiative decays, and lifetimes has been very slow. Experimentally, nearly all the branching fractions of the $\Lambda_{c}^{+}$are measured relative to the $p K^{-} \pi^{+}$mode. The recent measurements $\mathcal{B}\left(\Lambda_{c}^{+} \rightarrow p K^{-} \pi^{+}\right)=\left(6.84 \pm 0.24_{-0.27}^{+0.21}\right) \%$ by Belle and $(5.84 \pm 0.27 \pm 0.23) \%$ by BESIII are very encouraging. Moreover, BESIII recently measured 2-body, 3-body and 4-body nonleptonic decay modes of $\Lambda_{c}^{+}$with significantly improved precision. It is conceivable that many new data emerging from LHCb, BESIII, and B factories in the near future and from the experiments at J-PARC and PANDA in the future can be used to test the underlying mechanism of hadronic weak decays.

Acknowledgements I am grateful to Hai-Bo Li for inviting me to attend the International Workshop on Physics at Future High Intensity Collider @ 2-7 GeV in China held at University of Science and Technology of China on January 13-16, 2015. He urged me to write up the review on charmed baryons which will appear in the special topic of Frontiers of Physics, "Potential Physics at a Super Tau-Charm Factory". This research was supported in part by the Ministry of Science and Technology of R.O.C. under Grant No. 104-2112-M-001-022.

Open Access This article is distributed under the terms of the Creative Commons Attribution License which permits any use, distribution, and reproduction in any medium, provided the original author(s) and the source are credited.

\section{References}

1. H. Y. Cheng, Charm baryon production and decays, Int. J. Mod. Phys. A 24, Suppl. 1, 593 (2009) [arXiv: 0809.1869 [hep-ex]], Chap. 24

2. J. G. Körner, M. Krämer, and D. Pirjol, Heavy baryons, Prog. Part. Nucl. Phys. 33, 787 (1994) [arXiv: hep$\mathrm{ph} / 9406359]$

3. S. Bianco, F. L. Fabbri, D. Benson, and I. I. Bigi, A Cicerone for the physics of charm, Riv. Nuovo Cim. 26, \#7-8 (2003) [arXiv: hep-ex/0309021]

4. W. Roberts and M. Pervin, Heavy baryons in a quark model, Int. J. Mod. Phys. A 23, 2817 (2008) [arXiv: 0711.2492 [nucl-th]]

5. E. Klempt and J. M. Richard, Baryon spectroscopy, Rev. Mod. Phys. 82, 1095 (2010) [arXiv: 0901.2055 [hep-ph]]

6. V. Crede and W. Roberts, Progress towards understanding baryon resonances, Rept. Prog. Phys. 76, 076301 (2013) [arXiv: 1302.7299 [nucl-ex]]

7. A. J. Bevan, et al. [BaBar and Belle Collaborations], Charmed baryon spectroscopy and decays, Eur. Phys. J. C 74(11), 3026 (2014) [arXiv: 1406.6311 [hep-ex]], Chap. 19.4

8. K. A. Olive, et al. [Particle Data Group], The review of particle physics, Chin. Phys. C 38, 090001 (2014)

9. D. Pirjol and T. M. Yan, Predictions for $s$ wave and $p$ wave heavy baryons from sum rules and constituent quark model (1): Strong interactions, Phys. Rev. D 56, 5483 (1997) [arXiv: hep-ph/9701291]

10. H. Y. Cheng and C. K. Chua, Strong decays of charmed baryons in heavy hadron chiral perturbation theory, Phys. Rev. D 75, 014006 (2007) [arXiv: hep-ph/0610283]

11. S. H. Lee, et al. [Belle Collaboration], Measurements of the masses and widths of the $\Sigma_{c}(2455)^{0 /++}$ and $\Sigma_{c}(2520)^{0 /++}$ baryons, Phys. Rev. D 89, 091102 (2014) [arXiv: 1404.5389 [hep-ex]]

12. Y. Kato, et al. [Belle Collaboration], Search for doubly charmed baryons and study of charmed strange baryons at Belle, Phys. Rev. D 89, 052003 (2014) [arXiv: 1312.1026 [hep-ex]]

13. Y. Kato [Belle Collaboration], Search for doubly-charmed baryons and study of charmed-strange baryon states at Belle, PoS DIS 2014, 195 (2014) 
14. P. Cho, Strong and electromagnetic decays of two new $\Lambda_{c}^{*}$ baryons, Phys. Rev. D 50, 3295 (1994) [arXiv: hep$\mathrm{ph} / 9401276]$

15. M. Artuso, et al. [CLEO collaboration], Observation of new states decaying into $\Lambda_{c}^{+} \pi^{-} \pi^{+}$, Phys. Rev. Lett. 86, 4479 (2001)

16. Y. Oh and B. Y. Park, Excited states of heavy baryons in the Skyrme model, Phys. Rev. D 53, 1605 (1996)

17. S. Capstick and N. Isgur, Baryons in a relativized quark model with chromodynamics, Phys. Rev. D 34, 2809 (1986)

18. L. A. Copley, N. Isgur, and G. Karl, Charmed baryons in a quark model with hyperfine interactions, Phys. Rev. D 20, 768 (1979)

19. D. Ebert, R. N. Faustov, and V. O. Galkin, Masses of excited heavy baryons in the relativistic quark model, Phys. Lett. B 659, 612 (2008) [arXiv: 0705.2957 [hep-ph]]

20. B. Aubert, et al. [BaBar Collaboration], Observation of a charmed baryon decaying to $D^{0} p$ at a mass near 2.94 $\mathrm{GeV} / \mathrm{c}^{2}$, Phys. Rev. Lett. 98, 012001 (2007) [arXiv: hepex/0603052]

21. R. Mizuk, et al. [Belle Collaboration], Experimental constraints on the spin and parity of the $\Lambda_{c}(2880)^{+}$, Phys. Rev. Lett. 98, 262001 (2007) [arXiv: hep-ex/0608043]

22. C. Chen, X. L. Chen, X. Liu, W. Z. Deng, and S. L. Zhu, Strong decays of charmed baryons, Phys. Rev. D 75, 094017 (2007) [arXiv: 0704.0075 [hep-ph]]

23. X. H. Zhong and Q. Zhao, Charmed baryon strong decays in a chiral quark model, Phys. Rev. D 77, 074008 (2008) [arXiv: 0711.4645 [hep-ph]]

24. A. Selem, A Diquark Interpretation of the Structure and Energies of Hadrons, Senior thesis, MIT (2005)

25. A. Selem and F. Wilczek, Hadron systematics and emergent diquarks, arXiv: hep-ph/0602128

26. D. Ebert, R. N. Faustov, and V. O. Galkin, Spectroscopy and Regge trajectories of heavy baryons in the relativistic quark-diquark picture, Phys. Rev. D 84, 014025 (2011) [arXiv: 1105.0583 [hep-ph]]

27. X. G. He, X. Q. Li, X. Liu, and X. Q. Zeng, $\Lambda_{c}^{+}(2940)$ : A possible molecular state? Eur. Phys. J. C 51, 883 (2007) [arXiv: hep-ph/0606015]

28. H. Garcilazo, J. Vijande, and A. Valcarce, Faddeev study of heavy baryon spectroscopy, J. Phys. G 34, 961 (2007)

29. R. Mizuk, et al. [Belle Collaboration], Observation of an Isotriplet of Excited Charmed Baryons Decaying to $\Lambda_{c}^{+} \pi$, Phys. Rev. Lett. 94, 122002 (2005)

30. R. Chistov, et al. [Belle Collaboration], Observation of new states decaying into $\Lambda_{c}^{+} K^{-} \pi^{+}$and $\Lambda_{c}^{+} K_{S}^{0} \pi^{-}$, Phys. Rev. Lett. 97, 162001 (2006) [arXiv: hep-ex/0606051]

31. B. Aubert, et al. [BaBar Collaboration], A study of excited charm-strange baryons with evidence for new baryons $\Xi_{c}(3055)^{+}$and $\Xi_{c}(3123)^{+}$, Phys. Rev. D 77, 012002 (2008) [arXiv: 0710.5763 [hep-ex]]

32. H. Y. Cheng and C. K. Chua, Strong decays of charmed baryons in heavy hadron chiral perturbation theory: An update, arXiv: 1508.05653 [hep-ph]
33. B. Aubert, et al. [BaBar Collaboration], A study of $\bar{B} \rightarrow$ $\Xi_{c} \bar{\Lambda}_{c}^{-}$and $\bar{B} \rightarrow \Lambda_{c}^{+} \bar{\Lambda}_{c}^{-} \bar{K}$ decays at BABAR, Phys. Rev. $D$ 77, 031101 (2008) [arXiv: 0710.5775 [hep-ex]]

34. X. H. Guo, K. W. Wei, and X. H. Wu, Some mass relations for mesons and baryons in Regge phenomenology, Phys. Rev. D 78, 056005 (2008) [arXiv: 0809.1702 [hep-ph]]

35. B. Chen, K. W. Wei, and A. Zhang, Assignments of $\Lambda_{Q}$ and $\Xi_{Q}$ baryons in the heavy quark-light diquark picture, Eur. Phys. J. A 51, 82 (2015) [arXiv: 1406.6561 [hep-ph]]

36. H. X. Chen, W. Chen, Q. Mao, A. Hosaka, X. Liu, and S. L. Zhu, $P$-wave charmed baryons from QCD sum rules, Phys. Rev. D 91, 054034 (2015) [arXiv: 1502.01103 [hep-ph]]

37. S. M. Gerasyuta and E. E. Matskevich, Charmed $\left(70,1^{-}\right)$ baryon multiplet, Int. J. Mod. Phys. E 17, 585 (2008) [arXiv: 0709.0397 [hep-ph]]

38. L. H. Liu, L. Y. Xiao, and X. H. Zhong, Charm-strange baryon strong decays in a chiral quark model, Phys. Rev. D 86, 034024 (2012) [arXiv: 1205.2943 [hep-ph]]

39. B. Aubert, et al. [BaBar Collaboration], Observation of an excited charm baryon $\Omega_{c}^{*}$ decaying to $\Omega_{c}^{0} \gamma$, Phys. Rev. Lett. 97, 232001 (2006) [arXiv: hep-ex/0608055]

40. Y. B. Dong, A. Faessler, T. Gutsche, and V. E. Lyubovitskij, Charmed baryon $\Sigma_{c}(2800)$ as a $N D$ hadronic molecule, Phys. Rev. D 81, 074011 (2010) [arXiv: 1002.0218 [hep-ph]]

41. J. He and X. Liu, The observed charmed hadron $\Lambda_{c}(2940)^{+}$ and the $D^{*} N$ interaction, Phys. Rev. D 82, 114029 (2010) [arXiv: 1008.1500 [hep-ph]]

42. Y. Yamaguchi, S. Ohkoda, S. Yasui, and A. Hosaka, Hadronic molecules for charmed and bottom baryons near thresholds, Phys. Rev. D 87, 074019 (2013) [arXiv: 1301.4557 [hep-ph]]

43. Y. B. Dong, A. Faessler, T. Gutsche, and V. E. Lyubovitskij, Role of the hadron molecule $\Lambda_{c}(2940)$ in the $p \bar{p} \rightarrow$ $p D^{0} \bar{\Lambda}_{c}(2286)$ annihilation reaction, Phys. Rev. D 90, 094001 (2014) [arXiv: 1407.3949 [hep-ph]]

44. J. R. Zhang, $S$-wave $D^{(*)} N$ molecular states: $\Sigma_{c}(2800)$ and $\Lambda_{c}(2940)^{+}$? Phys. Rev. D 89, 096006 (2014) [arXiv: 1212.5325 [hep-ph]]

45. T. F. Carames and A. Valcarce, Multiquark contributions to charm baryon spectroscopy, Phys. Rev. D 90, 014042 (2014) [arXiv: 1507.08046 [hep-ph]]

46. M. Mattson, et al. [SELEX Collaboration], First observation of the doubly charmed baryon $\Xi_{c c}^{+}$, Phys. Rev. Lett. 89, 112001 (2002) [arXiv: hep-ex/0208014]

47. A. Ocherashvili, et al. [SELEX Collaboration], Confirmation of the double charm baryon $\Xi_{c c}^{+}(3520)$ via its decay to $p D^{+} K^{-}$, Phys. Lett. $B$ 628, 18 (2005) [arXiv: hepex/0406033]

48. S. P. Ratti [FOCUS Collaboration], New results on $c$-baryons and a search for cc-baryons in FOCUS, Nucl. Phys. Proc. Suppl. 115, 33 (2003)

49. B. Aubert, et al. [BaBar Collaboration], Search for doubly charmed baryons $\Xi_{c c}^{+}$and $\Xi_{c c}^{++}$in BABAR, Phys. Rev. D 74, 011103 (2006) [arXiv: hep-ex/0605075] 
50. R. Aaij, et al. [LHCb Collaboration], Search for the doubly charmed baryon $\Xi_{c c}^{+}$, JHEP 1312, 090 (2013) [arXiv: 1310.2538 [hep-ex]]

51. K. W. Wei, B. Chen, and X. H. Guo, Masses of doubly and triply charmed baryons, arXiv: 1503.05184 [hep-ph]

52. M. Karliner and J. L. Rosner, Baryons with two heavy quarks: Masses, production, decays, and detection, Phys. Rev. D 90, 094007 (2014) [arXiv: 1408.5877 [hep-ph]]

53. Z. G. Wang, Analysis of the $1 / 2^{-}$and $3 / 2^{-}$heavy and doubly heavy baryon states with QCD sum rules, Eur. Phys. J. A 47, 81 (2011) [arXiv: 1003.2838 [hep-ph]]

54. Z. G. Wang, Analysis of the $\frac{3}{2}^{+}$heavy and doubly heavy baryon states with QCD sum rules, Eur. Phys. J. C 68, 459 (2010) [arXiv: 1002.2471 [hep-ph]]

55. Z. G. Wang, Analysis of the $\frac{1}{2}^{+}$doubly heavy baryon states with QCD sum rules, Eur. Phys. J. A 45, 267 (2010) [arXiv: 1001.4693 [hep-ph]]

56. Z. F. Sun, Z. W. Liu, X. Liu, and S. L. Zhu, The masses and axial currents of the doubly charmed baryons, Phys. Rev. D 91, 094030 (2015) [arXiv: 1411.2117 [hep-ph]]

57. P. Perez-Rubio, S. Collins, and G. Bali, Charmed baryon spectroscopy and light flavour symmetry from lattice QCD, Phys. Rev. D 92, 034504 (2015) [arXiv: 1503.08440 [hep-lat]]

58. M. Padmanath, R. G. Edwards, N. Mathur, and M. Peardon, Spectroscopy of doubly-charmed baryons from lattice QCD, Phys. Rev. D 91, 094502 (2015) [arXiv: 1502.01845 [hep-lat]]

59. Z. S. Brown, W. Detmold, S. Meinel, and K. Orginos, Charmed bottom baryon spectroscopy from lattice QCD, Phys. Rev. D 90, 094507 (2014) [arXiv: 1409.0497 [hep-lat]]

60. C. Alexandrou, V. Drach, K. Jansen, C. Kallidonis, and G. Koutsou [ETM Collaboration], Baryon spectrum with $N_{f}=2+1+1$ twisted mass fermions, Phys. Rev. D 90, 074501 (2014) [arXiv: 1406.4310 [hep-lat]]

61. S. Basak, S. Datta, A. T. Lytle, M. Padmanath, P. Majumdar, and N. Mathur [ILGTI Collaboration], Hadron spectra from overlap fermions on HISQ gauge configurations, PoS LAT. 2013, 243 (2014) [arXiv: 1312.3050 [hep-lat]]

62. Y. Namekawa, et al. [PACS-CS Collaboration], Charmed baryons at the physical point in $2+1$ flavor lattice QCD, Phys. Rev. D 87, 094512 (2013) [arXiv: 1301.4743 [hep-lat]]

63. S. Durr, G. Koutsou, and T. Lippert, Meson and Baryon dispersion relations with Brillouin fermions, Phys. Rev. D 86, 114514 (2012) [arXiv: 1208.6270 [hep-lat]]

64. R. A. Briceno, H. W. Lin, and D. R. Bolton, Charmedbaryon spectroscopy from lattice QCD with $N_{f}=2+1+1$ flavors, Phys. Rev. D 86, 094504 (2012) [arXiv: 1207.3536 [hep-lat]]

65. L. Liu, H. W. Lin, K. Orginos, and A. Walker-Loud, Singly and doubly charmed $J=1 / 2$ baryon spectrum from lattice QCD, Phys. Rev. D 81, 094505 (2010) [arXiv: 0909.3294 [hep-lat]]

66. H. Na and S. A. Gottlieb, Charm and bottom heavy baryon mass spectrum from lattice QCD with $2+1$ flavors, PoS LAT. 2007, 124 (2007) [arXiv: 0710.1422 [hep-lat]]
67. K. U. Can, G. Erkol, M. Oka, and T. T. Takahashi, A look inside charmed-strange baryons from lattice $\mathrm{QCD}$, arXiv: 1508.03048

68. T. M. Yan, H. Y. Cheng, C. Y. Cheung, G. L. Lin, Y. C. Lin, and H. L. Yu, Heavy quark symmetry and chiral dynamics, Phys. Rev. D 46, 1148 (1992) [Erratum: Phys. Rev. D 55, 5851(E) (1997)]

69. M. B. Wise, Chiral perturbation theory for hadrons containing a heavy quark, Phys. Rev. D 45, 2188 (1992)

70. G. Burdman and J. Donoghue, Union of chiral and heavy quark symmetries, Phys. Lett. B 280, 287 (1992)

71. G. Chiladze and A. F. Falk, Phenomenology of new baryons with charm and strangeness, Phys. Rev. D 56, 6738 (1997) [arXiv: hep-ph/9707507]

72. A. E. Blechman, A. F. Falk, D. Pirjol, and J. M. Yelton, Threshold effects in excited charmed baryon decays, Phys. Rev. D 67, 074033 (2003) [arXiv: hep-ph/0302040]

73. Y. M. Yao, et al. [Particle Data Group], The review of particle physics, J. Phys. G 33, 1 (2006)

74. S. Tawfiq, P. J. O'Donnell, and J. G. Körner, Charmed baryon strong coupling constants in a light-front quark model, Phys. Rev. D 58, 054010 (1998)

75. M. A. Ivanov, J. G. Korner, V. E. Lyubovitskij, and A. G. Rusetsky, Strong and radiative decays of heavy flavored baryons, Phys. Rev. D 60, 094002 (1999) [arXiv: hep$\mathrm{ph} / 9904421]$

76. M. Q. Huang, Y. B. Dai, and C. S. Huang, Decays of excited charmed $\Lambda$-type and $\Sigma$-type baryons in heavy hadron chiral perturbation theory, Phys. Rev. D 52, 3986 (1995)

77. M. Q. Huang, Y. B. Dai, and C. S. Huang, Decays of excited charmed $\Lambda$-type and $\Sigma$-type baryons in heavy hadron chiral perturbation theory, Phys. Rev. D 55, 7317(E) (1997)

78. C. Albertus, Hernández, J. Nieves, and J. M. Verde-Velasco, Study of the strong $\Sigma_{c} \rightarrow \Lambda \pi, \Sigma_{c}^{*} \rightarrow \Lambda_{c} \pi$ and $\Xi_{c}^{*} \rightarrow \Xi_{c} \pi$ decays in a nonrelativistic quark model, Phys. Rev. D 72, $094022(2005)$

79. T. Aaltonen, et al. [CDF Collaboration], Measurements of the properties of $\Lambda_{c}(2595), \Lambda_{c}(2625), \Sigma_{c}(2455)$, and $\Sigma_{c}(2520)$ baryons, Phys. Rev. D 84, 012003 (2011) [arXiv: 1105.5995 [hep-ex]]

80. J. P. Alexander, et al. [CLEO Collaboration], Evidence of new states decaying into $\Xi_{c}^{*} \pi$, Phys. Rev. Lett. 83, 3390 (1999)

81. I. I. Bigi, N. G. Uraltsev, and A. I. Vainshtein, Nonperturbative corrections to inclusive beauty and charm decays: QCD versus phenomenological models, Phys. Lett. B 293, 430 (1992)

82. I. I. Bigi, N. G. Uraltsev, and A. I. Vainshtein, Nonperturbative corrections to inclusive beauty and charm decays: QCD versus phenomenological models, Phys. Lett. B 297, 477(E) (1992)

83. J. Chay, H. Georgi, and B. Grinstein, Lepton energy distributions in heavy meson decays from QCD, Phys. Lett. B 247, 399 (1990)

84. J. Chay and S. J. Rey, Instanton contribution to $B \rightarrow X_{\mu} e \bar{\nu}$ decay, Z. Phys. C 68, 431 (1995) 
85. M. E. Luke, Effects of subleading operators in the heavy quark effective theory, Phys. Lett. B 252, 447 (1990)

86. H. Y. Cheng, A Phenomenological analysis of heavy hadron lifetimes, Phys. Rev. D 56, 2783 (1997) [arXiv: hep$\mathrm{ph} / 9704260]$

87. V. V. Kiselev and A. K. Likhoded, Baryons with two heavy quarks, Phys. Usp. 45, 455 (2002) [Usp. Fiz. Nauk 172, 497 (2002)] [arXiv: hep-ph/0103169]

88. V. V. Kiselev, A. K. Likhoded, and A. I. Onishchenko, Lifetimes of doubly charmed baryons: $\Xi_{c c}^{+}$and $\Xi_{c c}^{++}$, Phys. Rev. D 60, 014007 (1999) [arXiv: hep-ph/9807354]

89. B. Guberina, B. Melic, and H. Stefancic, Inclusive decays and lifetimes of doubly charmed baryons, Eur. Phys. J. C 9, 213 (1999) [Erratum: Eur. Phys. J. C 13, 551 (2000)] [arXiv: hep-ph/9901323]

90. C. H. Chang, T. Li, X. Q. Li, and Y. M. Wang, Lifetime of doubly charmed baryons, Commun. Theor. Phys. 49, 993 (2008) [arXiv: 0704.0016 [hep-ph]]

91. M. Fukugita, T. Inami, N. Sakai, and S. Yazaki, Nonleptonic decays of kaons in the $1 / N_{c}$ expansion, Phys. Lett. B 72, 237 (1977)

92. D. Tadić and J. Trampetić, Weak meson decays and the $1 / N_{c}$ expansion, Phys. Lett. B 114, 179 (1982)

93. M. Bauer and B. Stech, Exclusive D Decays, Phys. Lett. B 152, 380 (1985)

94. A. J. Buras, J.-M. Gérard, and R. Rückl, $1 / N$ expansion for exclusive and inclusive charm decays, Nucl. Phys. B 268, 16 (1986)

95. B. Blok and M. Shifman, Towards a theory of weak hadronic decays of charmed particles, Sov. J. Nucl. Phys. 45, 135 (1987) [Yad. Fiz. 45, 211 (1987)]

96. B. Blok and M. Shifman, Weak nonleptonic decays of charmed mesons in QCD sum rules, Sov. J. Nucl. Phys. 45, 301 (1987) [Yad. Fiz. 45, 478 (1987)]

97. B. Blok and M. Shifman, Weak nonleptonic decays of charmed mesons: Theory versus experiment, Sov. J. Nucl. Phys. 45, 522 (1987) [Yad. Fiz. 45, 841 (1987)]

98. H. Y. Cheng and B. Tseng, Nonleptonic weak decays of charmed baryons, Phys. Rev. D 46, 1042 (1992)

99. H. Y. Cheng and B. Tseng, Nonleptonic weak decays of charmed baryons, Phys. Rev. D 55, 1697(E) (1997)

100. J. G. Körner and M. Krämer, Exclusive nonleptonic charm baryon decays, Z. Phys. C 55, 659 (1992)

101. J. G. Körner, G. Kramer, and J. Willrodt, Weak decays of the charmed baryon $C_{0}^{+}$and the inclusive yield of $\Lambda$ and $p$, Phys. Lett. B 78, 492 (1978)

102. J. G. Körner, G. Kramer, and J. Willrodt, Jet like distributions from the weak decay of heavy quarks, Z. Phys. C 2, 117 (1979)

103. B. Guberina, D. Tadić, and J. Trampetić, Hard and soft gluonic effects in charmed baryon decays, Z. Phys. C 13, 251 (1982)

104. F. Hussain and M. D. Scadron, Nonleptonic weak decays of charmed baryons, Nuovo Cimento A 79, 248 (1984)
105. F. Hussain and K. Khan, Current algebra and nonleptonic weak decays of charmed baryons, Nuovo Cimento A 88, 213 (1985)

106. R. E. Karlsen and M. D. Scadron, Charmed baryon decays, Europhys. Lett. 14, 319 (1991)

107. D. Ebert and W. Kallies, Nonleptonic decays of charmed baryons in the MIT bag model, Phys. Lett. B 131, 183 (1983)

108. D. Ebert and W. Kallies, Nonleptonic decays of charmed baryons in the MIT bag model, Phys. Lett. B 148, 502(E) (1984)

109. D. Ebert and W. Kallies, Bag model matrix elements of the parity violating weak hamiltonian for charmed baryons, Yad. Fiz. 40, 1250 (1984)

110. D. Ebert and W. Kallies, An application of a heavy quark bag model to nonleptonic decays of charmed baryons, $Z$. Phys. C 29, 643 (1985)

111. H. Y. Cheng, Remarks on the Lifetime of Charmed Baryon $\Lambda_{c}^{+}$, Z. Phys. C 29, 453 (1985)

112. Yu. L. Kalinovsky, V. N. Pervushin, G. G. Takhtamyshev, and N. A. Sarikov, Chiral Phenomenology of Weak Decays of Charmed Baryons, Sov. J. Part. Nucl. 19, 47 (1988)

113. S. Pakvasa, S. F. Tuan, and S. P. Rosen, Parity violation and flavor selection rules in charmed-baryon decays, Phys. Rev. D 42, 3746 (1990)

114. G. Kaur and M. P. Khanna, Two-body charmed-baryon weak decays, Phys. Rev. D 44, 182 (1991)

115. G. Kaur and M. P. Khanna, Charmed baryon weak decays in heavy quark effective theory, Phys. Rev. D 45, R3024 (1992)

116. G. Turan and J. O. Eeg, Contributions to $\Lambda_{c}^{+} \rightarrow \pi \Sigma, \pi \Lambda$ and $p \bar{K}^{0}$, Z. Phys. C 51, 599 (1991)

117. T. Uppal, R. C. Verma, and M. P. Khana, Constituent quark model analysis of weak mesonic decays of charm baryons, Phys. Rev. D 49, 3417 (1994)

118. H. Y. Cheng and B. Tseng, Cabibbo-allowed nonleptonic weak decays of charmed baryons, Phys. Rev. D 48, 4188 (1993)

119. Q. P. Xu and A. N. Kamal, Cabibbo-favored nonleptonic decays of charmed baryons, Phys. Rev. D 46, 270 (1992)

120. Q. P. Xu and A. N. Kamal, Nonleptonic charmed-baryon decays: $B_{c} \rightarrow B\left(3 / 2^{+}\right.$, decuplet $)+P\left(0^{-}\right)$or $V\left(1^{-}\right)$, Phys. Rev. D 46, 3836 (1992)

121. K. K. Sharma and R. C. Verma, A Study of weak mesonic decays of $\Lambda_{c}$ and $\Xi_{c}$ baryons on the basis of HQET results, Eur. Phys. J. C 7, 217 (1999) [arXiv: hep-ph/9803302]

122. P. Żenczykowski, Nonleptonic charmed-baryon decays: Symmetry properties of parity-violating amplitudes, Phys. Rev. D 50, 5787 (1994)

123. M. A. Ivanov, J. G. Korner, V. E. Lyubovitskij, and A. G. Rusetsky, Exclusive nonleptonic decays of bottom and charm baryons in a relativistic three quark model: Evaluation of nonfactorizing diagrams, Phys. Rev. D 57, 5632 (1998) [arXiv: hep-ph/9709372]

124. L. L. Chau, H. Y. Cheng, and B. Tseng, Analysis of two-body decays of charmed baryons using the quark diagram scheme, Phys. Rev. D 54, 2132 (1996) [arXiv: hep$\mathrm{ph} / 9508382]$ 
125. Y. Kohara, Quark-diagram analysis of charmed-baryon decays, Phys. Rev. D 44, 2799 (1991)

126. A. Zupanc, et al. [Belle Collaboration], Measurement of the Branching Fraction $\mathcal{B}\left(\Lambda_{c}^{+} \rightarrow p K^{-} \pi^{+}\right)$, Phys. Rev. Lett. 113, 042002 (2014) [arXiv: 1312.7826 [hep-ex]]

127. X. K. Zhou, Talk presented at Hadron 2015, September 1318, 2015, Newport News, USA

128. A. Contu, D. Fonnesu, R. G. C. Oldeman, B. Saitta, and C. Vacca, A method to measure the absolute branching fractions of $\Lambda_{c}$ decays, Eur. Phys. J. C 74, 3194 (2014) [arXiv: 1408.6802 [hep-ex]]

129. K. Abe, et al. [Belle Collaboration], Observation of Cabibbosuppressed and W-exchange $\Lambda_{c}^{+}$baryon decays, Phys. Lett. B 524, 33 (2002)

130. B. Aubert, et al. [BaBar Collaboration], Measurements of $\Lambda_{c}^{+}$branching fractions of Cabibbo-suppressed decay modes involving $\Lambda$ and $\Sigma^{0}$, Phys. Rev. D 75, 052002 (2007) [arXiv: hep-ex/0601017]

131. H. Y. Cheng and B. Tseng, $1 / M$ corrections to baryonic form factors in the quark model, Phys. Rev. D 53, 1457 (1996)

132. H. Y. Cheng and B. Tseng, $1 / M$ corrections to baryonic form factors in the quark model, Phys. Rev. D 55, 1697(E) (1997)

133. M. Bishai, et al. [CLEO Collaboration], Measurement of the decay asymmetry parameters in $\Lambda_{c}^{+} \rightarrow \Lambda \pi^{+}$and $\Lambda_{c}^{+} \rightarrow$ $\Sigma^{+} \pi^{0}$, Phys. Lett. B 350, 256 (1995)

134. J. M. Link, et al. [FOCUS Collaboration], Measurements of $\Xi_{c}^{+}$branching ratios, Phys. Lett. B 571, 139 (2003)

135. R. Dhir and C. S. Kim, Axial-vector emitting weak nonleptonic deacys of $\Omega_{c}^{0}$ Baryon, Phys. Rev. D 91, 114008 (2015) [arXiv: 1501.04259 [hep-ph]]

136. H. Y. Cheng, C. Y. Cheung, G. L. Lin, Y. C. Lin, T. M. Yan, and H. L. Yu, Heavy flavor conserving nonleptonic weak decays of heavy baryons, Phys. Rev. D 46, 5060 (1992)

137. M. B. Voloshin, Weak decays $\Xi_{Q} \rightarrow \Lambda_{Q} \pi$, Phys. Lett. B 476, 297 (2000) [arXiv: hep-ph/0001057]

138. X. Li and M. B. Voloshin, Decays $\Xi_{b} \rightarrow \Lambda_{b} \pi$ and diquark correlations in hyperons, Phys. Rev. D 90, 033016 (2014) [arXiv: 1407.2556 [hep-ph]]

139. S. Faller and T. Mannel, Light-quark decays in heavy hadrons, arXiv: 1503.06088 [hep-ph]

140. A. Chodos, R. L. Jaffe, K. Johnson, and C. B. Thorn, Baryon structure in the bag theory, Phys. Rev. D 10, 2599 (1974)

141. T. DeGrand, R. L. Jaffe, K. Johnson, and J. Kiskis, Masses and other parameters of the light hadrons, Phys. Rev. D 12, 2060 (1975)

142. R. Pérez-Marcial, R. Huerta, A. Garcia, and M. Avila-Aoki, Predictions for semileptonic decays of charm baryons. II. Nonrelativistic and MIT bag quark models, Phys. Rev. D 40, 2955 (1989)

143. R. Pérez-Marcial, R. Huerta, A. Garcia, and M. Avila-Aoki, Predictions for semileptonic decays of charm baryons. II. Nonrelativistic and MIT bag quark models, Phys. Rev. D 44, 2203(E) (1991)
144. R. Singleton, Semileptonic baryon decays with a heavy quark, Phys. Rev. D 43, 2939 (1991)

145. M. A. Ivanov, V. E. Lyubovitskij, J. G. Körner, and P. Kroll, Heavy baryon transitions in a relativistic three quark model, Phys. Rev. D 56, 348 (1997) [arXiv: hep-ph/9612463]

146. C. W. Luo, Heavy to light baryon weak form-factors in the light cone constituent quark model, Eur. Phys. J. C 1, 235 (1998)

147. R. S. Marques de Carvalho, et al., Form factors and decay rates for heavy $\Lambda$ semileptonic decays from QCD sum rules, Phys. Rev. D 60, 034009 (1999)

148. M. Q. Huang and D. W. Wang, Semileptonic decay $\Lambda_{c} \rightarrow$ $\Lambda \ell+\nu$ from QCD light-cone sum rules, arXiv: hep$\mathrm{ph} / 0608170$

149. T. Gutsche, M. A. Ivanov, J. G. Körner, V. E. Lyubovitskij, and P. Santorelli, Heavy-to-light semileptonic decays of $\Lambda_{b}$ and $\Lambda_{c}$ baryons in the covariant confined quark model, Phys. Rev. D 90, 114033 (2014) [arXiv: 1410.6043 [hep-ph]]

150. K. Azizi, Y. Sarac, and H. Sundu, Light cone QCD sum rules study of the semileptonic heavy $\Xi_{Q}$ and $\Xi_{Q}^{\prime}$ transitions to $\Xi$ and $\Sigma$ baryons, Eur. Phys. J. A 48, 2 (2012) [arXiv: 1107.5925 [hep-ph]]

151. M. Pervin, W. Roberts, and S. Capstick, Semileptonic decays of heavy lambda baryons in a quark model, Phys. Rev. C 72, 035201 (2005) [nucl-th/0503030]

152. J. W. Hinson, et al. [CLEO Collaboration], Improved measurement of the form-factors in the decay $\Lambda_{c}^{+} \rightarrow \Lambda e^{+} \nu_{e}$, Phys. Rev. Lett. 94, 191801 (2005) [hep-ex/0501002]

153. K. Azizi, M. Bayar, Y. Sarac, and H. Sundu, Semileptonic $\Lambda_{b, c}$ to nucleon transitions in full QCD at light cone, Phys. Rev. D 80, 096007 (2009) [arXiv: 0908.1758 [hep-ph]]

154. M. Pervin, W. Roberts, and S. Capstick, Semileptonic decays of heavy omega baryons in a quark model, Phys. Rev. C 74, 025205 (2006) [arXiv: nucl-th/0603061]

155. C. Albertus, E. Hernandez, and J. Nieves, Exclusive $c \rightarrow$ $s, d$ semileptonic decays of ground-state spin- $1 / 2$ doubly charmed baryons, Phys. Lett. B 704, 499 (2011) [arXiv: 1108.1296 [hep-ph]]

156. A. Faessler, T. Gutsche, M. A. Ivanov, J. G. Körner, and V. E. Lyubovitskij, Semileptonic decays of double heavy baryons in a relativistic constituent three-quark model, Phys. Rev. D 80, 034025 (2009) [arXiv: 0907.0563 [hep-ph]]

157. D. Ebert, R. N. Faustov, V. O. Galkin, and A. P. Martynenko, Semileptonic decays of doubly heavy baryons in the relativistic quark model, Phys. Rev. D 70, 014018 (2004)

158. D. Ebert, R. N. Faustov, V. O. Galkin, and A. P. Martynenko, Semileptonic decays of doubly heavy baryons in the relativistic quark model, Phys. Rev. D 77, 079903 (2008) [arXiv: hep-ph/0404280]

159. X. H. Guo, H. Y. Jin and X. Q. Li, Weak semileptonic decays of heavy baryons containing two heavy quarks, Phys. Rev. D 58, 114007 (1998) [arXiv: hep-ph/9805301]

160. H. Y. Cheng, C. Y. Cheung, G. L. Lin, Y. C. Lin, T. M. Yan, and H. L. Yu, Chiral Lagrangians for radiative decays of heavy hadrons, Phys. Rev. D 47, 1030 (1993) [arXiv: hepph/9209262] 
161. H. Y. Cheng, C. Y. Cheung, G. L. Lin, Y. C. Lin, T. M. Yan, and H. L. Yu, Corrections to chiral dynamics of heavy hadrons: SU(3) symmetry breaking, Phys. Rev. D 49, 5857 (1994)

162. H. Y. Cheng, C. Y. Cheung, G. L. Lin, Y. C. Lin, T. M. Yan, and H. L. Yu, Corrections to chiral dynamics of heavy hadrons: SU(3) symmetry breaking, Phys. Rev. D $55,5851(\mathrm{E})(1997)$

163. N. Jiang, X. L. Chen, and S. L. Zhu, Electromagnetic decays of the charmed and bottom baryons in chiral perturbation theory, Phys. Rev. D 92, 054017 (2015) [arXiv: 1505.02999 [hep-ph]]

164. H. Y. Cheng, Remarks on the strong coupling constants in heavy hadron chiral Lagrangians, Phys. Lett. B 399, 281 (1997) [hep-ph/9701234]

165. J. Dey, M. Dey, V. Shevchenko and P. Volkovitsky, Radiative decays of $S$ wave charmed baryons, Phys. Lett. B 337, 185 (1994)

166. S. Tawfiq, J. G. Körner, and P. J. O'Donnell, Electromagnetic transitions of heavy baryons in the $S U\left(2 N_{f}\right) \otimes O(\overline{3})$ symmetry, Phys. Rev. D 63, 034005 (2001)

167. M. J. Savage, E2 strength in the radiative charmed baryon decay $\Sigma_{c}^{*} \rightarrow \Lambda_{c} \gamma$, Phys. Lett. B 345, 61 (1995)

168. M. C. Baũls, A. Pich, and I. Scimemi, Electromagnetic decays of heavy baryons, Phys. Rev. D 61, 094009 (2000)

169. T. M. Aliev, K. Azizi, and A. Ozpineci, Radiative decays of the heavy flavored baryons in light cone QCD sum rules, Phys. Rev. D 79, 056005 (2009) [arXiv: 0901.0076 [hep-ph]]

170. Z. G. Wang, Analysis of the vertexes $\Omega_{Q}^{*} \Omega_{Q} \phi$ and radiative decays $\Omega_{Q}^{*} \rightarrow \Omega_{Q} \gamma$, Phys. Rev. D 81, 036002 (2010) [arXiv: 0909.4144 [hep-ph]]

171. Z. G. Wang, Analysis of the vertexes $\Xi_{Q}^{*} \Xi_{Q}^{\prime} V, \Sigma_{Q}^{*} \Sigma_{Q} V$ and radiative decays $\Xi_{Q}^{*} \rightarrow \Xi_{Q}^{\prime} \gamma, \Sigma_{Q}^{*} \rightarrow \Sigma_{Q} \gamma$, Eur. Phys. J. A 44, 105 (2010) [arXiv: 0910.2112 [hep-ph]]

172. A. Bernotas and V. Šimonis, Radiative M1 transitions of heavy baryons in the bag model, Phys. Rev. D 87, 074016 (2013) [arXiv: 1302.5918 [hep-ph]]

173. A. Majethiya, B. Patel, and P. C. Vinodkumar, Radiative decays of single heavy flavour baryons, Eur. Phys. J. A 42, $213(2009)$
174. H. Bahtiyar, K. U. Can, G. Erkol, and M. Oka, $\Omega_{c} \gamma \rightarrow \Omega_{c}^{*}$ transition in lattice QCD, Phys. Lett. B 747, 281 (2015) [arXiv: 1503.07361 [hep-lat]]

175. M. Lu, M. J. Savage, and J. Walden, The radiative charmed baryon decay $\Xi_{c 2}^{0 *} \rightarrow \Xi_{c 1}^{0} \gamma$, Phys. Lett. B 369, 337 (1996)

176. Z. Aziza Baccouche, C. K. Chow, T. D. Cohen, and B. A. Gelman, Excited heavy baryons and their symmetries. 3. Phenomenology, Nucl. Phys. A 696, 638 (2001) [arXiv: hepph/0105148]

177. S. L. Zhu, Strong and electromagnetic decays of $p$ wave heavy baryons $\Lambda_{c 1}, \Lambda_{c 1}^{*}$, Phys. Rev. D 61, 114019 (2000) [hep-ph/0002023]

178. C. K. Chow, Radiative decays of excited $\Lambda_{Q}$ baryons in the bound state picture, Phys. Rev. D 54, 3374 (1996) [arXiv: hep-ph/9510421]

179. D. Gamermann, C. E. Jimenez-Tejero, and A. Ramos, Radiative decays of dynamically generated charmed baryons, Phys. Rev. D 83, 074018 (2011) [arXiv: 1011.5381 [hep-ph]]

180. Y. B. Dong, A. Faessler, T. Gutsche, S. Kumano, and V. E. Lyubovitskij, Radiative decay of $\Lambda_{c}(2940)^{+}$in a hadronic molecule picture, Phys. Rev. D 82, 034035 (2010) [arXiv: 1006.4018 [hep-ph]]

181. T. Branz, A. Faessler, T. Gutsche, M. A. Ivanov, J. G. Korner, V. E. Lyubovitskij, and B. Oexl, Radiative decays of double heavy baryons in a relativistic constituent threequark model including hyperfine mixing, Phys. Rev. D 81, 114036 (2010) [arXiv: 1005.1850 [hep-ph]]

182. A. N. Kamal and Riazuddin, Four-quark operators in hyperon radiative decays and Hara's theorem, Phys. Rev. D 28,2317 (1983)

183. A. N. Kamal and R. C. Verma, Radiative weak decays of baryons, Phys. Rev. D 26, 190 (1982)

184. L. C. Hua, Quark-diagram analysis of hyperon radiative decays: General formulation, Phys. Rev. D 26, 199 (1982)

185. H. Y. Cheng, C. Y. Cheung, G. L. Lin, Y. C. Lin, T. M. Yan, and H. L. Yu, Effective Lagrangian approach to weak radiative decays of heavy hadrons, Phys. Rev. D 51, 1199 (1995) [arXiv: hep-ph/9407303]

186. T. Uppal and R. C. Verma, Weak electromagnetic decays of charm baryons, Phys. Rev. D 47, 2858 (1993) 